\title{
Embodiment in Socially Interactive Robots
}

\author{
Eric Deng \\ University of Southern California \\ denge@usc.edu
}

\author{
Bilge Mutlu \\ University of Wisconsin-Madison \\ bilge@cs.wisc.edu
}

\author{
Maja J Matarić \\ University of Southern California \\ mataric@usc.edu
}

December 3, 2019

\begin{abstract}
Physical embodiment is a required component for robots that are structurally coupled with their real-world environments. However, most socially interactive robots do not need to physically interact with their environments in order to perform their tasks. When and why should embodied robots be used instead of simpler and cheaper virtual agents?

This paper reviews the existing work that explores the role of physical embodiment in socially interactive robots. This class consists of robots that are not only capable of engaging in social interaction with humans, but are using primarily their social capabilities to perform their desired functions. Socially interactive robots provide entertainment, information, and/or assistance; this last category is typically encompassed by socially assistive robotics. In all cases, such robots can achieve their primary functions without performing functional physical work.

To comprehensively evaluate the existing body of work on embodiment, we first review work from established related fields including psychology, philosophy, and sociology. We then systematically review 65 studies evaluating aspects of embodiment published from 2003 to 2017 in major peer-reviewed robotics publication venues. We examine relevant aspects of the selected studies, focusing on the embodiments compared, tasks evaluated, social roles of robots, and measurements. We introduce three taxonomies for the types of robot embodiment, robot social roles, and human-robot tasks. These taxonomies are used to deconstruct the design and interaction spaces of socially interactive robots and facilitate analysis and discussion of the reviewed studies. We use this newly-defined methodology to critically discuss existing works, revealing topics within embodiment research for social interaction, assistive robotics, and service robotics, in which more extensive exploration would greatly improve the current understanding of the impact of embodiment on human perception and evaluation of human-robot interactions.

The introduced taxonomy for embodiment design is used as a starting point for outlining our characterization of the design space of robot embodiments. The presented
\end{abstract}


characterization can be used to discuss how the physical embodiment of socially interactive robots relates to social capabilities and affordances. By introducing a general model of the design space, existing research findings can better advise robot designers and we discuss how these findings can inform researchers through design decisions in the development of future socially interactive robots.

Keywords: Embodiment, Human-Robot Interaction, Social Robotics, Product Design, Human-Computer Interaction, Service Robots, Reporting Guidelines, Methodology

\section{Introduction}

As technology development and sophistication continue to progress at an ever-growing rate, automated systems are quickly becoming integrated into everyday life. These systems have assisted humans in tasks ranging from scheduling (Blum and Langley, 1997), ordering food deliveries (Simmons et al., 1997), entertaining guests (Breazeal, 2004), enhancing assembly line work (Simmons et al., 2001), and coaching physical and mental health activities (Langen et al., 1994).

A growing subset of these technologies are artificial agents, whether they be on-screen, in virtual reality $(\mathrm{VR})$, or physically embodied. We are witnessing parallel and synergistic growth of the core technologies of artificial intelligence, computing, and manufacturing, all facilitating the development of interactive artificial agents. Researchers and engineers working in human-robot interaction (HRI) and socially interactive robotics are designing, building, testing, and deploying robots that interact with humans and perform a wide range of tasks (Goodrich and Schultz, 2007) as partners in a growing number of domains including manufacturing (Asfahl, 1992), healthcare (Inoue et al., 2008; Robins et al., 2006; Wada and Shibata, 2006; Werry et al., 2001; Nikolopoulos et al., 2011), education (Saerbeck et al., 2010; Greczek et al., 2014; Clabaugh et al., 2015; Takeuchi et al., 2006; Kanda et al., 2004; Gordon et al., 2015), and entertainment(Kidd and Breazeal, 2004; Shinozaki et al., 2008; Pereira et al., 2008; Klamer et al., 2010).

As these robots are interacting with users through primarily non-physical means, it is critical for them to be able to engage in effective social interactions. Embodiment provides the opportunity to leverage more channels of communication, including proxemics (Takayama and Pantofaru, 2009; Mead and Matarić, 2016; Mead et al., 2013), oculesics (Mutlu et al., 2012; Andrist et al., 2012,?), and gestures (Breazeal et al., 2005; Sidner et al., 2005) to enhance communication and the perception of being more trustworthy (Reilly, 1996), helpful (Reilly, 1996), and engaging(Kidd and Breazeal, 2004) than disembodied agents.

Although embodiment is a defining feature of robotics, the study of embodiment and embodied behavior predates robotics and extends well beyond it; it spans many fields of 
study, including neuroscience (Edelman, 2004), philosophy (Hendriks-Jansen, 1996), and social sciences (Gover, 1996; Kant and Jaki, 1981).

How critical is the physical embodiment of a robot in human-machine interaction? Embodiment is clearly a necessity for robots that physically interact with and manipulate objects, but most socially interactive robots do not physically interact with the environment to achieve their goals (Lee et al., 2004; Fong et al., 2003). As a result, in such contexts, the benefits of physical embodiment over less expensive and complex virtual presence is less obvious (Holz et al., 2009). This work explores the embodiment hypothesis in socially interactive robotics: "the hypothesis that physical embodiment has a measurable effect on performance and perception of social interactions" (Wainer et al., 2006).

Research in human communication and psychology has explored both physical and virtual embodied cues as tools for improving social interaction, including gaze behavior (Bailenson et al., 2001), head movements (Bailenson and Yee, 2006), and the persona effect (Moundridou and Virvou, 2002): the affective impact of artificial agents in social interaction. Kantian philosophy introduced the concepts of the mind-body and subjectobject problems in relation to the embodied view in the mid-1700's (Gover, 1996; Kant and Jaki, 1981), leading to the development of the "modern" embodiment hypothesis outlined by Ortega and Gasset (2010), Heidegger (1973), and Merleau-Ponty et al. (2004) and (Fieser and Dowden, 2011; Benner, 1994). Embodied cognition spans these fields, bringing together the work of Brooks (1990) and Moravec (1988a) in robotics and sensing, the modern-day philosophy of Clark (2008, 2007) and Hendriks-Jansen (1996), and research in neuroscience and biology from Edelman (2004), Longo et al. (2008), Damasio (1999), and Rosch et al. (1991). In human-computer interaction, non-physical interactions with artificial agents in social interactions have been studied (Cassell et al., 1999), specifically exploring the design of such systems for social abilities and quality of interactions they can produce (Rehnmark et al., 2005; Krämer, 2005). In robotics, specific dimensions of social interaction have been explored, as has the influence of the design of physical embodiment on interaction (Wainer et al., 2007), engagement (Kidd and Breazeal, 2004; Takayama and Pantofaru, 2009; Walters et al., 2005), trust (Bickmore and Cassell, 2001; Bickmore and Picard, 2005), and the perception of an agent (Burgoon et al., 2000; Jung and Lee, 2004; Kidd and Breazeal, 2004; Takayama and Pantofaru, 2009; Wainer et al., 2006; Walters et al., 2005).

Previous work in robotics suggests physical embodiment can increase engagement and enjoyment in social interactions with humans (Bainbridge et al., 2011; Kidd and Breazeal, 2004; Wainer et al., 2006, 2007). This paper presents a thorough review of existing work and analyzes existing results and approaches to embodiment to determine the current state of the embodiment hypothesis. As research continues to validate the importance of embodiment in socially interactive robots, the implications on robot design will become more apparent, 
because both the theoretical and practical importance of physical embodiment for humanrobot interactions translates into real-world applications through appropriate embodiment design. In this meta-review, we study various robotic platforms, most of which were designed for research uses, and then adapted to task-specific applications within research studies. We explore these embodiments and approaches (Mutlu et al., 2012) to collecting data toward quantifying the subjective qualities of the robot's physical embodiment. We then describe our characterization of the design space for socially interactive robots toward informing both future designers and researchers.

The rest of this paper is organized as follows. We first discuss the definition of embodiment in relevant fields of study, review past work in related fields, and introduce terminology for the rest of the paper. We then introduce a taxonomy of robot embodiments that provides the contexts for human-robot interactions in the surveyed studies. We then discuss the current state of the embodiment hypothesis in socially interactive robots based on the existing body of work, provide suggestions of areas that need further exploration, and recommend approaches that aid in the design of more structured studies. Finally, we introduce a characterization of the design space of socially interactive robots, discuss how different components of a robot's design relate to aspects of social interaction, and present an approach to leveraging existing research to design or select robot embodiments for future work.

\section{What is Embodiment?}

Embodiment is a fundamental concept studied in philosophy, psychology, neuroscience, communications, and engineering (Csordas, 1994; Goodwin, 2000; Csordas, 1990). In this chapter, we review how embodiment is treated in these fields to better understand the past, present, and future of the concept as it relates to socially interactive robotics.

\subsection{Embodiment in Philosophy and Ethics}

Embodied, or situated, cognition is a concept derived from embodiment in philosophy and ethics, a well-studied area in the humanities that spans topics such as social interaction, social influence, and decision-making (Shapiro, 2010). Wilson (2002) and Anderson (2003) discussed embodied cognition as an approach to examining the human experience being impacted by "aspects of the body beyond the brain."

In philosophy, cognition is seen as being critically influenced by all aspects of an agent's body, and the discussion of embodiment in that context is focused on the agent's sensorimotor capabilities (Rosch et al., 1991). For example, Wilson and Foglia (2011) attributed an agent's "beyond-the-brain body" as playing a critical role in that agent's cognitive processes. 
Embodiment is closely related to the agent's various expectations and limitations. All agents are in some way constrained by their embodiment; they are also highly dependent on affordances, "the fundamental properties of a device that determine its way of use", which are themselves derived from embodiment (Gibson, 1982). The affordances, expectations, and limitations set by an agent's embodiment are further discussed in Section 4.

The ethics of embodiment in social interaction relate to these affordances stemming from a robot's design. Interactive robots are often designed with the goals of being engaging and assistive. The robot's quality of being engaging aids interaction, but can also potentially lead to undesirable influence, unrealistic expectations, and perceived deception, disappointment, or emotional discomfort. Attachment toward the robot can develop, so that the removal of the robot may lead to grief and anxiety (Passman and Weisberg, 1975). Misleading embodiment design can also engender inappropriate use that can potentially lead to emotional or physical injury (Norman, 1999).

Classical works in philosophy establish the foundation for embodiment in general, including robot embodiment, and ethics further warns of negative consequences of some design choices.

\subsection{Embodiment in Psychology and Communication}

Scholars in the fields of psychology and human communications have long pondered the question of how and to what extent different media can be used to represent the real world. A significant body of literature discusses virtual reality (Merchant et al., 2014; Saposnik et al., 2011), perceived reality (Jussim, 1991; Jackson and Schuler, 1985), pictorial realism (Welch et al., 1996), and other related topics. Increasingly, communications researchers are becoming interested in presence and its relationship to embodiment (Biocca, 1997; Mantovani and Riva, 1999; Lombard and Ditton, 1997). Effective design of an embodied robot serves to increase its social presence and desired affordances.

Recent embodiment research in human communications fields has focused on presence in virtual reality platforms (Klein, 2003) and telepresence (Kim and Biocca, 1997; Durlach and Slater, 2000), building on the classical works (Gunawardena and Zittle, 1997; Gunawardena, 1995). There have also been further explorations of physical embodiment in social agents (Jung and Lee, 2004; Leyzberg et al., 2012; Kidd and Breazeal, 2004). In the next section, we discuss robotics embodiment studies whose results support the importance of social presence in both human-human and human-robot interaction. 


\subsection{Embodiment in Robotics and Design}

Research in artificial software agents encompasses virtual agents, relatable agents, affective agents, and most recently chatbots, and has been focused on the development of communicative systems that are physically disembodied, such as text interfaces (Looije et al., 2010), animations (Bartneck et al., 2004), or high-fidelity virtual characters (DeVault et al., 2014). The value of physical embodiment of artificial agents comes from the improvements seen in the interactions held between such agents and their human interaction partners. Two basic questions arise: (1) do physically embodied agents interact more effectively than their non-physically embodied counterparts? and (2) if so, why?

Rosch et al. (1991) discussed the influence that sensorimotor capabilities have on an agent's relationship with its environment-providing a richer experience for the agent and allowing the agent to exist in a richer context that bridges biology, psychology, and culture. Brooks (2002) drew parallels from this philosophical generalization of embodiment to the field of robotics. The sensorimotor capabilities of biological beings and robots, at a high level, affect the agent in very similar ways: the sensors and effectors define limitations to the ability of the agent to sense, manipulate, and navigate its environments. Biological agents have brains, muscles, and nerves that communicate on a network that enables the system to function. When discussing the embodiment, or "physical instantiation", of robots, we focus on the bodily presence of those machines. This includes the internal and external mechanical structures, embedded sensors, and motors that allow them to interact with the world around them (Brooks, 2002, 1990). All components of embodiment are inherently tied to the agent's function, whether the agent is biological or artificial.

Traditionally, roboticists were largely focused on the functional properties of physical embodiment, such as locomotion (Furusho and Masubuchi, 1986; Brooks, 1989; Wang and Thorpe, 2002), manipulation (Mason and Salisbury Jr, 1985; Rivin, 1987), and haptics (Lee and Nicholls, 1999; Fu et al., 1987; Moravec, 1988b). Human-robot interaction (HRI) is a relatively new and rapidly growing area of robotics that focuses on interaction with people in a broad variety of settings, and fundamentally changes how value is attributed to different components in robotic systems (Lee et al., 2006; Dym et al., 2005; Stickdorn et al., 2011). For instance, in HRI, the value of a gripper goes beyond its capabilities for manipulation to its role in communication: having independently-controlled fingers allows a robot hand to gesture in more complex ways and therefore opens doors to a broader realm of interactions. The value associated with socially interactive capabilities has stimulated new robot embodiments that are not capable of traditional functions (such as Pepper, Kiwi, and Cozmo), shown in Figure 2.1).

Designing for interaction rather than physical function fundamentally changes the nature of robot design. In Section 2, we provide a characterization of this new design space. As a first step, we define embodiment in the context of socially interactive robots. 


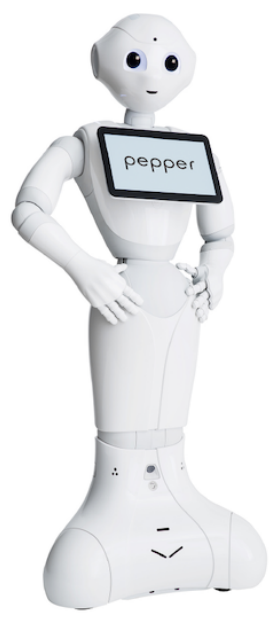

(a)

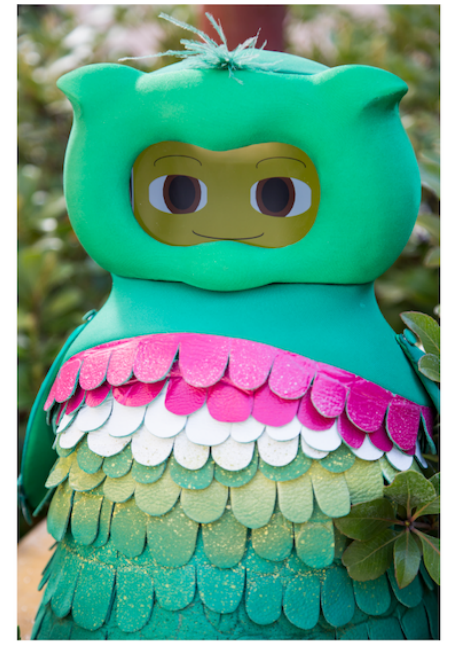

(b)

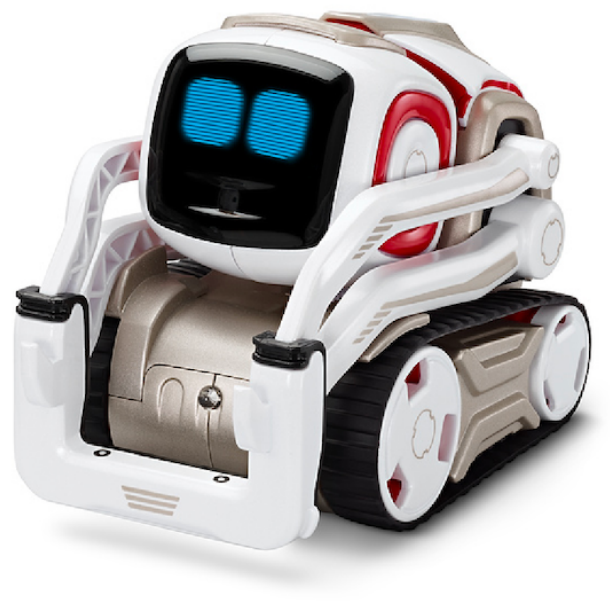

(c)

Figure 2.1: Embodied Socially Interactive Robot Platforms-(a) Softbank Pepper, (b) Spritebot Kiwi, (c) Anki Cozmo

\section{Defining Embodiment for Interactive Agents}

Embodiment in the context of artificial social agents has been a topic of discussion since the late 1900s-Zlatev (1997) explored situated embodiment, Sharkey and Ziemke (2001) studied mechanistic and phenomenal embodiment, Ziemke (1999) addressed natural embodiment, and Barsalou et al. (2003) discussed the concept of social embodiment, among many others.

Ziemke (2003) introduced six different "notions" of embodiment:

1. Structural Coupling: The physical coupling between the agent and its environment, based on the work of Maturana and Varela (1991, 1987). Quick et al. (1999) provided a definition of embodiment related to structural coupling:

System $\mathrm{X}$ is embodied in an environment $\mathrm{E}$ if perturbatory channels exist between the two. That means, $\mathrm{X}$ is embodied in $\mathrm{E}$ if for every time $\mathrm{t}$ at which both $\mathrm{X}$ and $\mathrm{E}$ exist, some subset of E's possible states with respect to $\mathrm{X}$ have the capacity to perturb X's state, and some subset of X's possible states with respect to E have the capacity to perturb E's state.

2. Historical Embodiment: The inherent relationships of any agent's embodiment with its history, especially in the context of adaptation, evolution, and growth (Valera et al., 1991; Riegler, 2002; Ziemke, 1999). 
3. Physical Embodiment: The physical instantiation of an agent in its environment, adapted into the concept of "physical grounding" (Brooks, 1990) which argues that "it is necessary to connect [intelligent systems] to the world via a set of sensors and actuators."

4. Organismoid Embodiment: The notion that cognition in an embodied artificial agent is, to some degree, dependent on its similarities to organismic counterparts.

5. Organismic Embodiment: The concept that "cognition is not only limited to physical, organism-like bodies, but in fact to organisms, i.e., living bodies" (Ziemke, 2003).

6. Social Embodiment: The idea that the embodiment of a socially interactive agent plays a significant role in social interactions. Barsalou et al. (2003) described social embodiment as "states of the body, such as postures, arm movements, and facial expressions, arise during social interaction and play central roles in social information processing." This is the notion of embodiment more relevant to the work in this paper, as it relates most to physical embodiment of socially interactive robots.

Quick et al. (1999) discussed embodiment in the context of structural coupling, addressing how embodiment is presented independent of any ontological context. This notion of embodiment, inspired by the interactions of Eschericha coli (E. coli) and its environment, is most concerned with the structural or physical relationships between the agent and its surrounding environment.

This work focuses on how the physical relationship between a socially interactive robot and its surrounding environment relate to the robot's sociability and presence. We adhere to the definition of embodiment that is a combination of the concepts of "social embodiment" and "situated structural coupling" from Ziemke (2003) and Quick et al. (1999), respectively.

In the following sections, we review the work related to embodiment in research areas outside of robotics, and discuss how they relate to the design and implementation of physical embodiment of socially interactive robots.

\subsubsection{Virtual Artificial Agents}

Virtual artificial agents generally fall into one of two categories: (1) immersed virtual reality or (2) on-screen virtual characters (Hillis, 1999). Virtual artificial agents and socially interactive robotics share several enabling technologies, including machine vision, speech, AI, and machine learning (Gratch et al., 2015). They also share related theoretical grounding, including psychology and sociology theories; Persson et al. (2001) presented a user-centered viewpoint of socially interactive agents, research that aims not to simulate social intelligence but to give the impression of the agent being socially intelligent. Taylor (2002) explored 
presence, social integration, and communication in virtual worlds with secondary charactersall critical aspects of embodiment.

The research in virtual agents has shown a significant need for embodiment in virtual social interactions (Ruhland et al., 2015). Many studies supplement qualitative interviews and observer notes with quantitative data (Biocca and Nowak, 2001; Smith and Harrison, 2001; Dautenhahn, 2001), such as toward understanding ownership of sub-components of embodiment(Kilteni et al., 2012) and administering POMS questionnaire before and after completing an activity(Garau et al., 2005). Virtual agents have been shown in research experiments to be engaging to a variety of populations (Cassell, 2001; Gratch et al., 2015), and have been developed for applications in education (Wagner et al., 2006; Traum et al., 2008), collaboration (Perlin and Goldberg, 1996; Vinayagamoorthy et al., 2004; Richard et al., 2001; Vosinakis and Panayiotopoulos, 2001), social skill training (Chollet et al., 2015), and post-traumatic stress disorder (Rizzo et al., 2010) and depression therapy (DeVault et al., 2014).

Work in virtual agents has repeatedly demonstrated the positive effects of embodied cues, such as gestures and expression (Scherer et al., 2012), in maintaining user engagement in both short-term and long-term interactions (Kose-Bagci et al., 2009; Bickmore and Picard, 2005; Bickmore and Cassell, 2005). Such embodied cues are shared aspects of virtual agents and socially interactive robots and have been shown to be transferable (Ono et al., 2001).

\subsubsection{Collaborative Robots}

Virtual agents can enable copresence, but physical robots enable colocation, which, in turn, can enable collaboration. As HRI expands, physical collaboration between people and machines is a major target of research and applications, ranging from manufacturing to the service sector.

Robots were originally envisioned for performing the three "Ds": dirty, dull, and dangerous work (Murphy, 2000). One of the first uses of robots at scale was in manufacturing and automation. Because of the predictability and repeatability of tasks on the assembly line, robots were designed for and placed in environments not accessed by human workers, or were caged for safety. Recent research and technological advancements have enabled the development of robot systems and control algorithms for deployment in manufacturing settings where people and robots share the same environment and work together to accomplish common goals (Nikolaidis and Shah, 2013). This development initially focused on intuitive interfaces and communication tools for master-slave relationships between operators and robots in teleoperational situations (Strabala et al., 2013; Mainprice and Berenson, 2013), but such systems required trained professionals to operate them and had marginal impact on the efficiency and safety of industrial workplaces. 
To allow for less trained users to effectively interact with and leverage industrial robot systems, HRI researchers have been working on various approaches to human-robot collaboration, such as using cross-training to improve task sharing between human and robot workers Nikolaidis and Shah (2013), planning shared work plans taking human ergonomics into consideration Pearce et al. (2018), and adapting robot actions to human motion, availability, adaptability, and intent Huang and Mutlu (2016); Lasota and Shah (2015); Huang et al. (2015b); Nikolaidis et al. (2017).

\subsubsection{Service and Socially Interactive Robots}

Concurrently with collaborative robotics, the broad area of service robotics has been growing rapidly, developing robots that can provide services in everyday life, such as vacuuming and cleaning floors (Jones, 2006; Forlizzi and DiSalvo, 2006; Mutlu and Forlizzi, 2008), folding laundry (Osawa et al., 2006; Maitin-Shepard et al., 2010), delivering packages (Simmons et al., 1997; Coltin and Veloso, 2014), giving museum tours (Nourbakhsh et al., 1999), driving autonomously (Levinson et al., 2011), and providing aid to special needs populations in the context of socially assistive robotics (Feil-Seifer and Mataric, 2005; Bemelmans et al., 2012; Broekens et al., 2009), along with numerous other uses.

As robots move from cages and from behind closed doors into shared spaces with humans, is has become critical to integrate social capabilities and new design considerations into the embodiments of those systems. Some considerations are related to safety, such as hiding pinch points and adding in physical compliance, and others to practical usability, such as height adjustment (Haddadin et al., 2009; Wyrobek et al., 2008; Matthias et al., 2011).

Similar to collaborative robots, socially interactive robots also need to be designed to be minimally intrusive, but their embodiments are used as tools for communication, acceptance, and engagement. These robots primarily interact through their social capabilities in order to achieve their goals. Accordingly, they must be able to both perceive (Rani et al., 2004; Kennedy et al., 2007; Bauer et al., 2008; Scherer et al., 2012) and generate communicative signals (Ono et al., 2001; Huang and Mutlu, 2014) that their human counterparts are able to intuitively understand, relate to, and accept. These requirements mean a fundamental change in the way robot embodiments are designed.

\section{Social Performance and Social Presence in Embodied Robots}

The combined ability of an artificial agent to generate and understand verbal and non-verbal communication can be organized into the following seven human social characteristics that can greatly improve a robot's social acceptance (Fong et al., 2003):

1. Express emotion

2. Communicate with high-level dialogue 
3. Learn/recognize models of other agents

4. Establish/maintain social relationships

5. Use natural cues (gaze, gestures, etc.)

6. Exhibit distinctive personality and character

7. Learn/develop social competencies

These components of social interaction tie into the concept that Lee et al. (2006) referred to as social presence, a key component in the success of social interactions. Studies have shown that physically-embodied agents possess social presence to a greater extent than their virtual counterparts (Shinozawa et al., 2003; Heerink et al., 2010).

There are a few different definitions of social presence across related research communities (HCI, communications, etc.); we adhere to the definition by Bainbridge et al. (2011) that defines social presence as "the degree to which a person's perceptions of an agent or robot shape social interaction with that robot". This concept is then broken down into two classes of design: "embodiment" and "co-location". Each class has attributes for creating rich, social interactions; in this paper we focus on exploring the physical embodiment of artificial agents.

The embodiment hypothesis in socially interactive robotics (Wainer et al., 2006) argues that a robot's physical presence augments its ability to generate rich communication. The physical embodiment of social agents provides them with more modes of communication that can be used to convey internal states and intentions in more intuitive, human-like ways (Lohan et al., 2010). Barsalou et al. (2003) concisely outlined four significant ways in which physical embodiment directly effects the social capabilities of these interactive systems:

First, perceived social stimuli do not just produce cognitive states, they produce bodily states as well. Second, perceiving bodily states in others produces bodily mimicry in the self. Third, bodily states in the self produce affective states. Fourth, the compatibility of bodily states and cognitive states modulates performance effectiveness.

\section{Types of Socially Interactive Robots}

Socially interactive robots vary in many aspects of embodiment and social ability. They can be classified into seven categories according to Fong et al. (2003), expanding on Breazeal (2003):

1. Socially Evocative: Robots that evoke feelings stemming from the natural human tendency to nurture and care for anthropomorphized agents. 
2. Social Interface: Robots that use social cues and communication modalities familiar to human users. This requires embodiments to be capable of generating (and often also understanding) those social cues.

3. Socially Receptive: Robots that are socially passive but benefit through interaction. They are limited in the social cues they are capable of learning by their respective embodiments.

4. Sociable: Robots that proactively interact with humans to complete internal goals.

5. Socially Situated: Robots in a social environment that they are capable of understanding and reacting to (Dautenhahn, 2002).

6. Socially Embedded: Robots that are socially situated but also structurally coupled with their environment and have knowledge of human interactional structures.

7. Socially Intelligent: Robots that have human-level social intellect. This is be the most complex and technologically-capable class of socially interactive robots.

\subsection{Summary}

In this section we discussed the bodies of work surrounding the concept of embodiment that are relevant to the field of socially interactive robots. Embodiment has been studied by a wide variety of disciplines. Philosophers have examined embodiment as a lens to the human experience, studied its relationship to human cognition, and discussed how it serves as a source for both physical and cognitive human social expectations. Psychologists and communications theorists have long been fascinated by the notion of presence and how symbolic representation of agents can be appropriately designed. Embodiment is inherently contextual; consequently, the latest developments in communication technology and media, such as virtual reality and on-screen characters, have had a strong influence on recent studies. Since human-robot interaction is a relatively young are of robotics, the value of embodiment in social HRI is also a relatively new area of study. Traditionally, the physical embodiment of robots has been discussed in the context of functional value-perception, mobility, and manipulation. We discussed how the field has now advanced to include considerations of interactive value and design affordances. We then discussed the related fields of virtual agents, collaborative robots, and service robots, all of which have relevant aspects of embodiment. Finally, we introduced the embodiment hypothesis that is fundamental to human-robot interaction.

Researchers of embodiment have various opinions on its role in an artificial agents' abilities-some see limited benefits from physical embodiment (Hoffmann and Krämer, 2013) while others claim that "intelligence cannot merely exist in the form of an abstract algorithm 
but requires a physical instantiation, a body" (Pfeifer and Scheier, 2001). In the next section, we review a set of embodiment studies conducted in socially interactive robotics over the last decade and a half, in order to evaluate the state of the embodiment hypothesis today.

\section{The Design Space for Socially Interactive Robots}

In design practice, formally defining a design space - the elements that designers can vary to create possible variations in the appearance, behavior, and overall makeup of a system - can facilitate constructive discussion and systematic experimentation. Robots are complex interactive systems, and defining a design space for them can guide future development and serve as a framework for understanding prior research and identifying gaps in our knowledge. The exploration of design space for socially interactive robots typically involves industrial design, animation, and interaction design to create variations in the physical construction, behavior, and interactive capabilities of robot systems. The physical design and appearance of socially interactive robots are inextricably tied to behavioral capabilities and interactivity, as they set user expectations and mental models regarding functional and social abilities of the robot Goetz et al. (2003); Lee et al. (2005). Furthermore, a set of contextual factors, such as the features of the task in which users are expected to interact with the robot and the role that the robot is envisioned to play in the task, shape user perceptions of the physical and behavioral characteristics of the robot and their interaction with it. Therefore, the design space for socially interactive robots must be defined in a way that integrates physical, behavioral, interactive, and contextual factors.

\subsection{Contextual Factors}

Interaction, whether between humans or between humans and robots, is always shaped by context. In the following subsections, we introduce a taxonomy for the two core contextual factors of interaction with socially interactive robots: the tasks in which robots are used and the roles that the robots play in those tasks.

\subsubsection{Tasks}

The first of the two dimensions of social context is the task at hand. Using the Circumplex Model for group tasks proposed by Mcgrath (1995) (seen in Figure 3.1), we classify the reviewed studies into one of eight octants along two main dimensions of (1) generatenegotiate and (2) execute-choose. The task at hand acts as the underlying driver of these interactions and is the more general of the two contextual factors being considered. Below, we define these eight task categories and provide example studies of each from our review. 


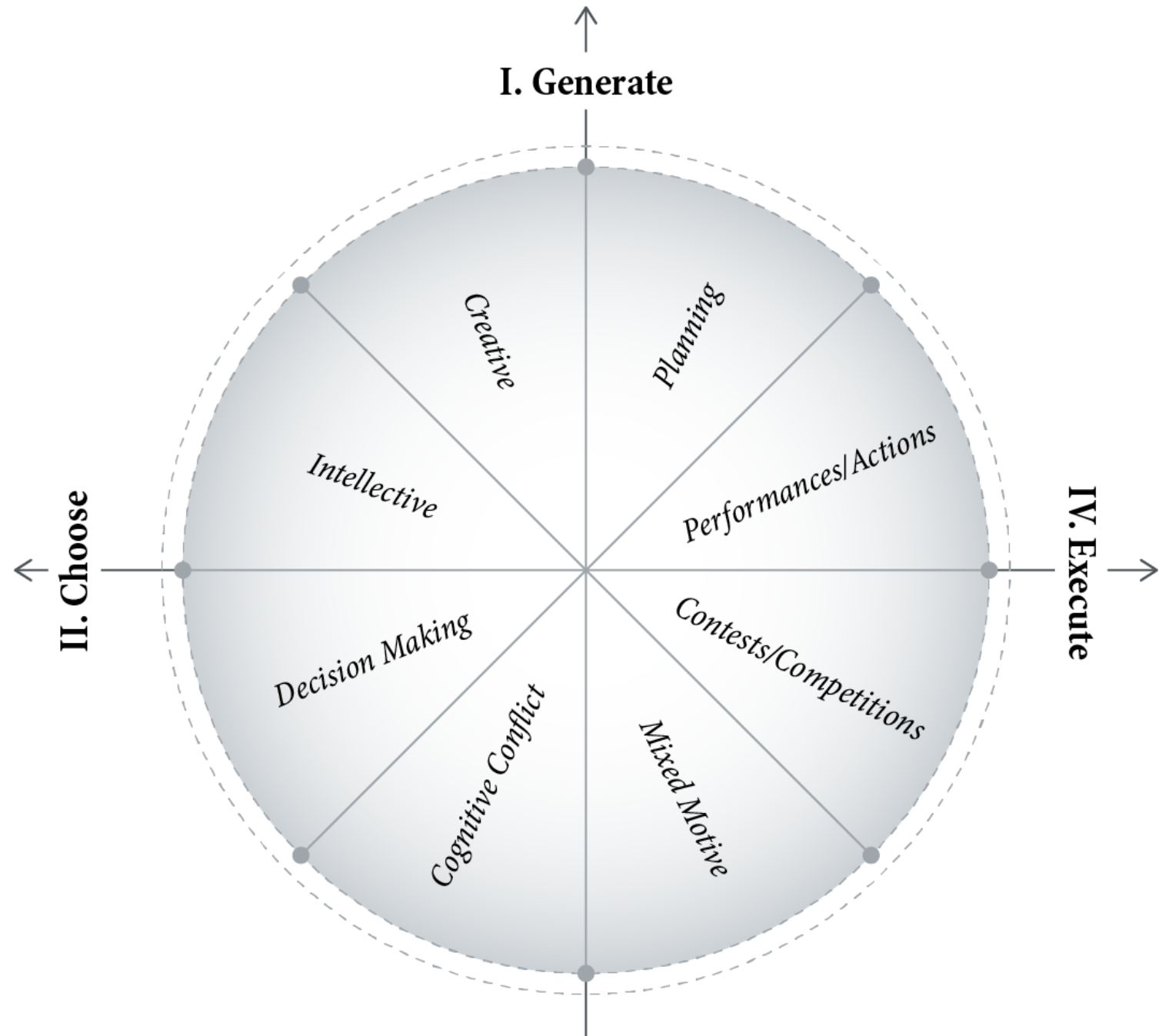

III. Negotiate

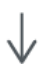

Figure 3.1: McGrath's (Mcgrath, 1995) Circumplex of Group Tasks, segmented into octants along the dimensions of generate-negotiate and execute-choose.

- Planning (Generate-Execute): Planning tasks are those in which a series of steps is determined by the interacting agents in order to reach a goal for the group. For example, Vossen et al. (2009) compared the influence of feedback relative to energy consumption used by an embodied robot and a computer. Users were asked to use 
a simulated washing machine interface to clean clothes while trying to minimize electricity consumption.

- Performances/Action (Execute-Generate): Performance or action tasks are those in which some or all members of the interaction group execute a series of actions, typically following a set of predetermined instructions, to achieve a goal. The action(s) taken depend on the task context, but the tasks share the characteristic of having quantifiable performance metrics. For example, Bainbridge et al. (2011) evaluated the use of a physically or virtually embodied artificial agent in instructing participants to perform tasks that ranged from moving stacks of books from one shelf to another to discarding stacks of books by placing them into a trash can.

- Contests/Competition (Execute-Negotiate): Contest tasks involve conflicts of power between interaction agents in action-based, competitive tasks (Posner et al., 2005). The competitive components involve negative-sum or zero-sum games (Nash, 1951) and thus associate negative cost (both social and functional) with task-related decisions. For example, Bartneck (2003) asked participants to compete against robot agents in a negotiation task involving stamps that were assigned values prior to the start of the game. Both the robot and the participant were trying to maximize their individual scores and could negotiate and trade with one another throughout the activity.

- Mixed-motive (Negotiate-Execute): Mixed motive tasks involve resolving conflicts of interest among interacting agents (Posner et al., 2005). Such conflicts are structured as positive sum games, in which the net benefits received by an individual party do not necessarily detract from the benefits of another (Nash, 1951). For example, Shinozawa et al. (2003) explored the use of robotic agents in retail settings with relevant social goals such as conversing with participants about purchasing a set of kitchen knives.

- Cognitive conflict (negotiate-choose): Cognitive conflict tasks involve resolving conflicts of viewpoints among interacting agents (Posner et al., 2005). For example, Pereira et al. (2008) used the iCat robot to play chess with participants, starting from a predetermined mid-game position with the participant at a slight advantage.

- Decision Making (Choose-Negotiate): Decision-making tasks are those in which interacting agents decide issues with no unique correct answer (Posner et al., 2005). For example, Lee et al. (2015) asked participants to rate the "genuineness" of smiles in artificial agents and robots by comparing Duchenne and non-Duchenne smiles.

- Intellective (Choose-Generate): Intellective tasks are similar to decision making tasks but have correct answers. For example, Zlotowski (2010) had participants solve math problems with the robot agent as a medium for feedback related to the task. 


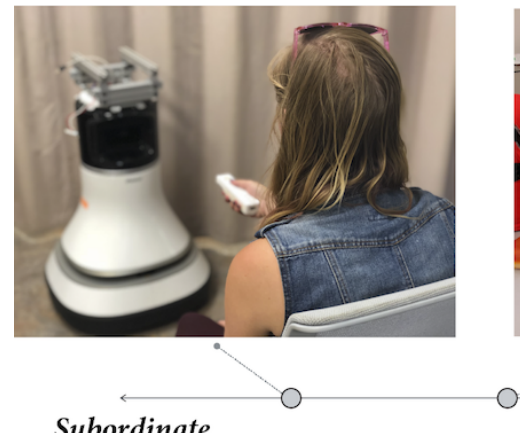

Subordinate

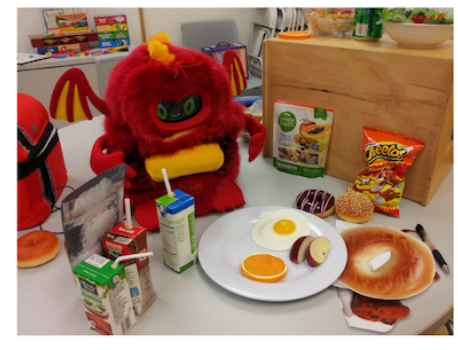

Peer

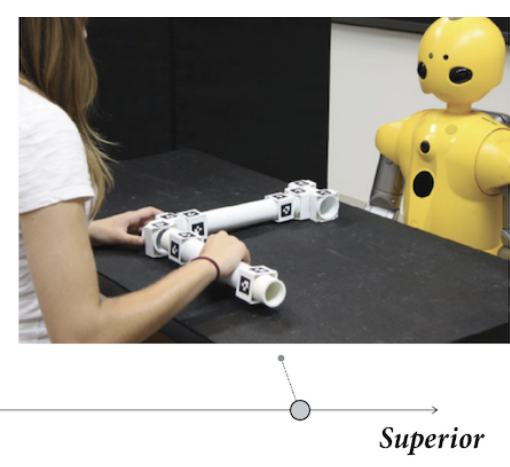

Superior

Figure 3.2: Examples of robot applications in different social roles, including a subordinate mobile base following remote controls, a peer eating "buddy" for children, and a superior robot "instructor" that gives the user task directions.

- Creative (generate-choose): Creative tasks involve generating ideas. While painting, composing, and photography are examples of typical creative tasks, in the context of Mcgrath (1995) task circumplex, those tasks are classified as performance tasks given that actions are being taken. In contrast, Fischer et al. (2012) is an example of a creative task that asked participants to describe objects to the robot that were selected by the experimenter.

\subsubsection{Social Roles}

The second dimension of context is the role the agent plays in the interaction. Roles are inherently tied to the agent's abilities to achieve certain contextualized goals, both social and task-oriented. For example, agents in the role of a "superior" may be capable of delivering trustworthy information and gaining adherence because of their perceived reliability and competence (Kennedy et al., 2015); those perceived as "peers" may facilitate interesting and engaging cognitive competition; and "subordinate" agents may improve user self-efficacy and encourage attachment formation through a balance of demonstrated ability and disclosed incompetence (Bartneck, 2003). Understanding how people respond to agents of varying social roles is critical for designing socially interactive robots. To discuss these roles, we look to classical works in organizational theory (Magee and Galinsky, 2008), plotting roles of agents along the spectrum that spans from subordinate to superior (Figure 3.2).

\subsection{Design Paradigms}

The second defining feature of socially interactive agents is the design of their embodiments, or their industrial design. The form that the agent's embodiment takes - physical, virtual, or disembodied — and the potential benefits of that form are key design considerations. 

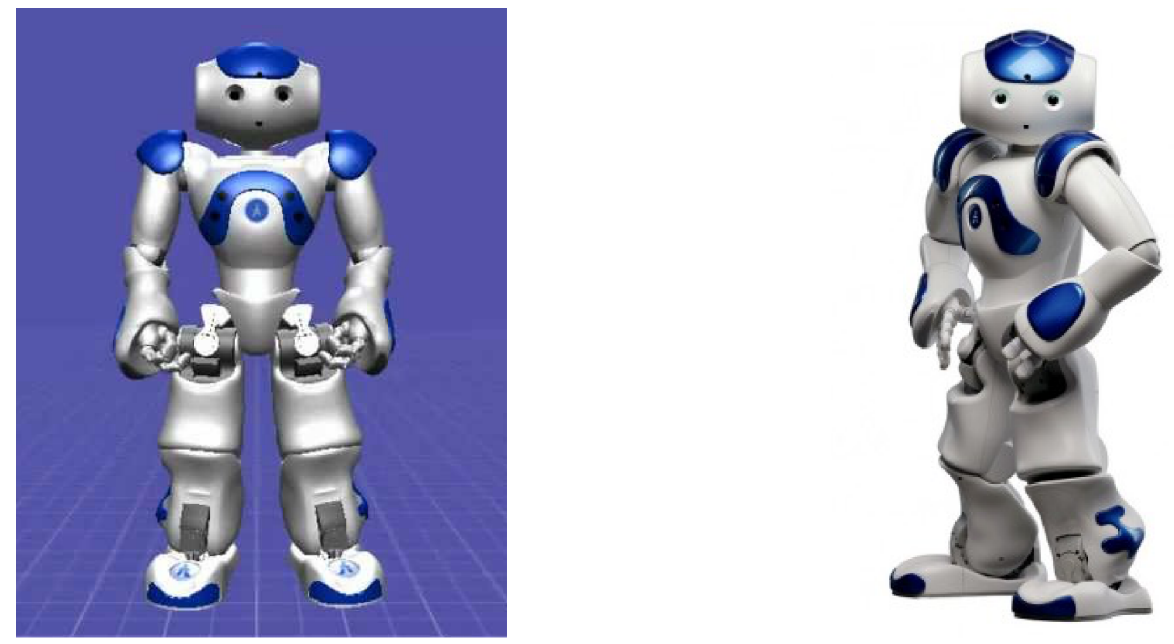

Figure 3.3: A virtual NAO robot (left) and a physical NAO robot (right), representing the virtual and physical or weak and strong embodiments.

Some researchers have characterized different forms of embodiment along the "weak" to "strong" axis Duffy and Joue (2000). Mutlu (2017) argued that the choice of virtual or physical representation goes beyond a weak vs. strong sense of embodiment to elicit disparate frames of min d and result in vastly different user experiences. Virtual embodiments bring users into the agent's environment, invite them to participate in a crafted narrative, provide proxemic relationships that are constrained and determined by physical arrangements and conventions, and offer a safe setting to experience emotions. Physical embodiments, on the other hand, are co-situated in the users' environment, perceived as independent agents pursuing their own goals, and seen as real-world, self-relevant stimuli. Interactions with physical embodiments emerge through joint action and intention, and proxemic relationships with these agents are dynamic and co-managed to follow human norms (Mead and Matarić, 2016, 2017). Despite these significant differences in the nature of interactions with virtual and physical embodiments, the body of work that we review here considers the form of embodiment to be a design choice and seeks to establish the differences in interaction outcomes through direct comparison.

Comparing physically embodied robots to their virtual counterparts (Figure 3.3) to test the value of physical embodiment in artificial agents is a common theme in the reviewed research. However, research on embodiment in socially interactive robotics also attempts to learn about specific design features and methods that may be used to create more engaging and effective robot systems. The design space for the embodiment of robots and virtual agents is vast. Because there are so many features of a robot's embodiment, the robots and virtual agents used in the reviewed studies vary greatly in their designs. To address the 
Figure 3.4: A characterization of the design of embodiments for artificial agents. Designs follow discrete metaphors but vary along a continuous axis of abstraction.

variability of embodiment design, we focus on two dimensions of every robot's design: (1) design metaphor and (2) level of abstraction, characterized in Figure 3.4.

\subsubsection{Design Metaphors}

The notion of the design metaphor stems from traditional design fields and refers to the design inspiration of an artifact, or in our case, robot. The metaphor for a robot's embodiment affords certain expectations for interaction partners and scaffolds social interactions. For instance, a humanoid robot with a mouth is more likely to be expected to speak compared to a bird-like robot with a beak. The design metaphors for socially interactive robots cover a wide range of possibilities, including cats, dogs, people, and cars. Since there is no simple linear relationships between these different metaphors (i.e., the metaphor of a cat is not obviously somewhere between the metaphor for a dog and a human), we define this subset of the robot design space as a discrete, nonlinear space. Because the design of embodiments can be inspired by multiple metaphors, we classify each embodiment by its primary design metaphor and discuss its level of abstraction relative to that singular design metaphor.

\subsubsection{Abstraction and Stylization}

The level of abstraction at which the design metaphor is manifested on the robot's embodiment defines how known abilities and characteristics from the design metaphor elicit expectations about the robot's capabilities. An example of differences in abstraction for the same design metaphor can be seen in Figure 3.5; all three robots are inspired by the human form and inherit varied subsets of human embodiment features such as arms, eyes, and mouth. The robot on the left, Kuri, looks much more abstract than the robot in the middle, Bandit, and the robot on the right, Mesmer, is much more human-realistic than the other two. Because of the differences in abstraction of their human-inspired forms, perceptions of these robots will differ and can affect the performance of each robot in different task scenarios. 

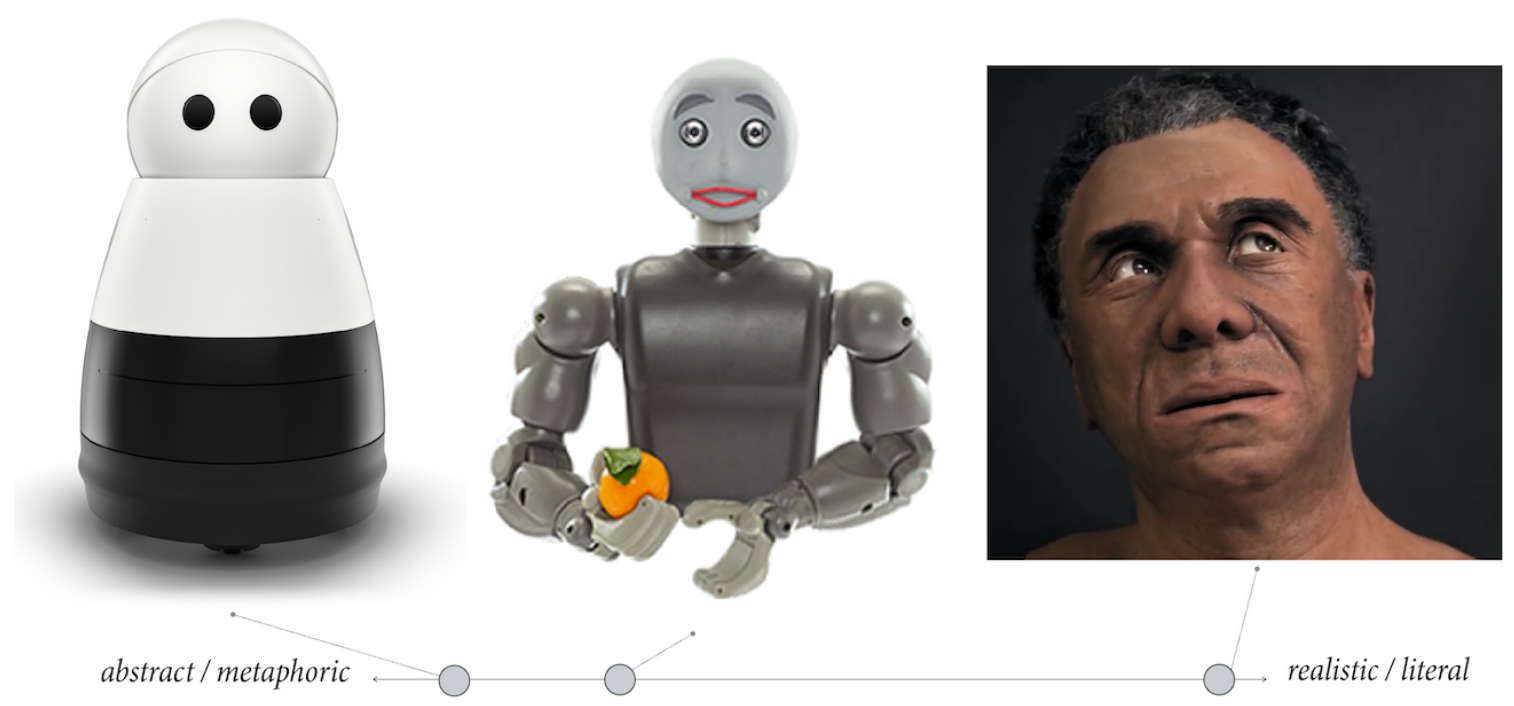

\section{Human Design Metaphor}

Figure 3.5: Three example robot embodiments spanning the spectrum of abstraction for the anthropomorphic/human design metaphor: Kuri (left), Bandit (middle), and Engineered Art's Mesmer (right).

\subsection{Behavior Design}

Human-robot interaction is grounded in human-human interaction and multi-modal communication patterns. Embodied robots can leverage rich channels of communication that are unavailable to purely text- or speech-based interactive systems. Work in human communication has provided evidence that embodied interaction, when effectively executed, can elicit improved performance in various social, cognitive, and task outcomes (Antle, 2009; Lee et al., 2012). In embodied interaction, agents utilize behavioral mechanisms that encompass both the ability to perform specific behavioral elements and the timing with which these behaviors are used in the context of the interaction. We refer to these behavioral elements as embodied cues Mutlu (2011). This section provides an overview of embodied cues used by agents in the reviewed studies or explored in human communication, focusing on cues that we believe will be important design variables for creating effective socially interactive robots.

\subsubsection{Limb-Based Gestures}

A large subset of embodied cues consists of hand, arm, and head movements, which - when used according to the norms of human 


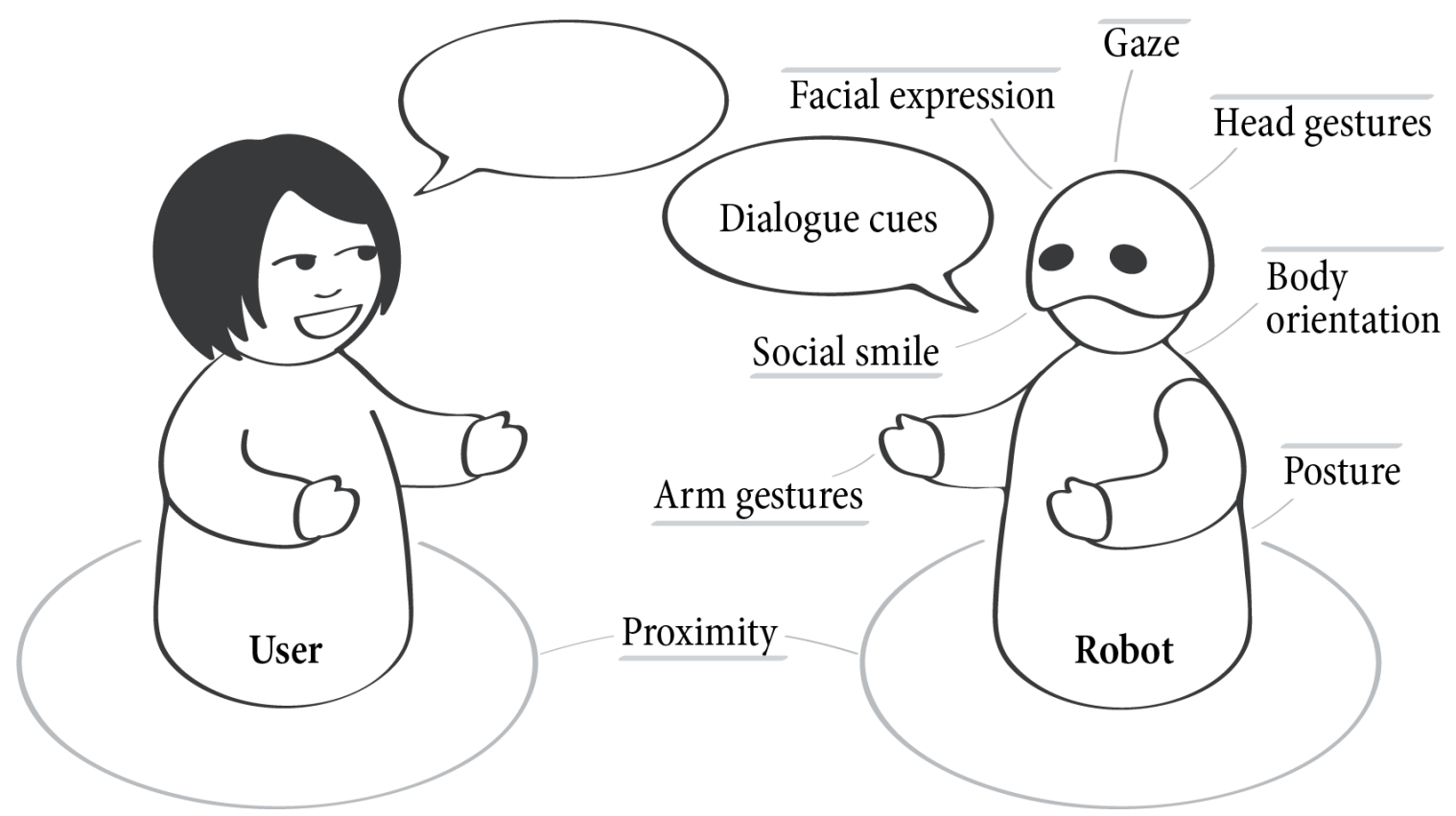

Figure 3.6: Some of the behavior design variables for socially interactive robots (adapted from (Mutlu, 2011)).

communication - can communicate a wide range of ideas and create rich and salient interactions (Krauss, 1998; McClave, 2000; Deng and Matarić, 2018). Those limb-based gestures fall into five primary categories: iconic, metaphoric, beat, cohesive, and deictic gestures (McNeill, 2008).

Iconic gestures are used to communicate ideas directly related to the semantics of the associated speech, while metaphoric gestures are used to communicate more abstract concepts and ideas. Both use "pictorial representations" commonly expressed through hand and arm movements (Krauss, 1998). Iconic gestures range from sign languages, which explicitly and specifically convey assigned semantics of the communicator's messages, to more general gestures that convey less specific meaning, such as "large." Beat gestures are related to physical representations of prosody and pace of speech and are often used to emphasize specific segments in speech and to maintain timing and pace during the interaction. These gestures can involve a wide variety of motions, including repetitive hand, arm, head, or full-body movements. Cohesive gestures are used to associate thematically related segments of speech and improve coherence and clarity of speech. Using similar gestures at targeted points during a verbal presentation helps observers to construct relationships between ideas being presented. Deictic gestures, or pointing gestures, are used to provide references and direct attention toward objects in the shared environment. These gestures are performed 

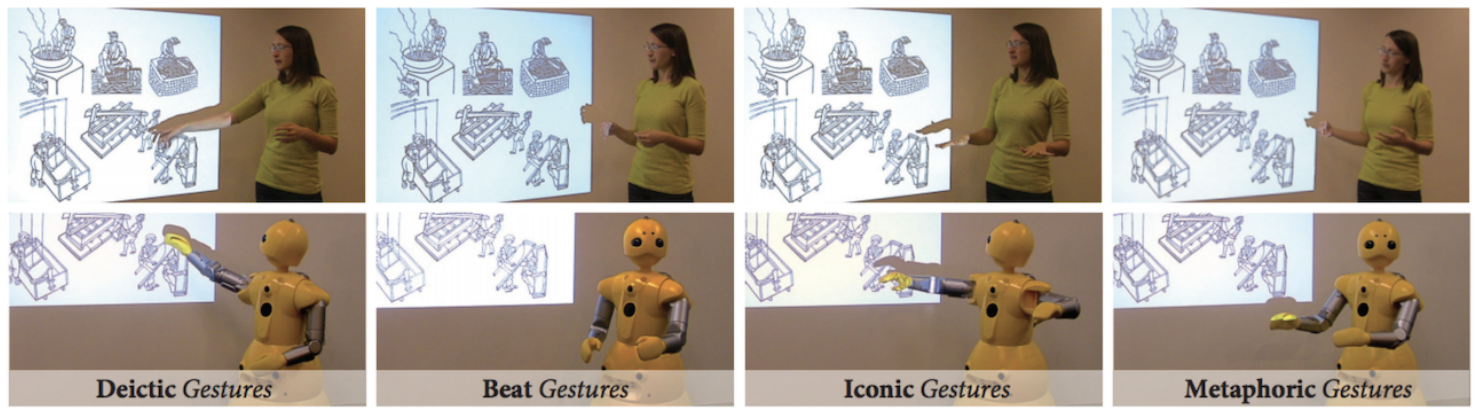

Figure 3.7: Examples of various limb-based gestures of embodied robots from work by Huang and Mutlu (2013).

with arm, hand, or head movements and serve as cues for establishing joint attention in situated interactions.

Head movements commonly serve as deictic, cohesive, and beat gestures. Speakers use them in the form of pointing or directional cues; listeners use them in the form of nods and shakes (Mutlu, 2011) to signal understanding and agreement, as back-channel cues for attention and uncertainty, and as a means of pacing interactions (Deng and Matarić, 2017).

\subsubsection{Posture}

The embodied cues discussed above are explicitly performed using different combinations of embodied features at specific times during an interaction (Knapp et al., 2013; Tomasello, 2010). The overall poses of the agent's body in its "resting state" are also important, as they provide cues about attitude and status relationships in the interaction (Mehrabian, 1969). By observing the overall orientation and the kinematic configuration of the agent, researchers have shown significant correlations between posture and speech, allowing prediction of upcoming speech from video (McQuown and Bateson, 1971). Posture cues, such as the "arms-akimbo position" where a communicator places the hands on the hips and bows the elbows outwardly, convey information about the internal state of the communicator, shaping how others perceive the communicator (Osborn, 1996; Mutlu, 2011). Researchers have studied these phenomena and developed systems that enable socially interactive robots to better interpret, and therefore generate, explicit posture cues (Gaschler et al., 2012). These outcomes highlight the importance of posture in the embodiment design space.

Posture, like limb-based gestures, are particularly affected by differences in robot embodiments. Different robot hardware inherently constrains embodied expressive gestures in different ways; mapping semantic gestures across different forms of embodiments is an important open challenge for generalizable expressions for socially interactive robots (Wang et al., 2006; Tosun et al., 2014). 


\subsubsection{Gaze}

The gaze cues of an individual, defined by the orientation - and shifts thereof - of the eyes, the head, and the body, convey rich information about the direction of attention and mental and emotional states of the individual Frischen et al. (2007). These cues serve a range of social functions, including facilitating turn-taking Duncan (1972); Mutlu et al. (2012), helping to establish joint attention Emery (2000), and signaling the intent and mental states of others Calder et al. (2002); Byom and Mutlu (2013); Huang et al. (2015a). The wide range of functions that gaze serves is due largely to their highly contextualized nature. For example, the aversion of gaze during turn-taking can help speakers to more effectively manage conversational roles, while listeners can use gaze aversion to regulate intimacy and put the speaker at ease Andrist et al. (2013, 2014). Gaze cues also serve as a supplement to or a replacement for deictic gestures Sato et al. (2009), providing speakers with the ability to direct attention toward objects in the environment Frischen et al. (2007), and to disambiguate what is being referred to in the environment Hanna and Brennan (2007); Huang and Mutlu (2012). Through gaze cues, individuals can signal personality Andrist et al. (2015), mental states Calder et al. (2002), and affect Mason et al. (2005). When used effectively, these cues can significantly enhance interaction outcomes, such as improved recall of information (Mutlu et al., 2006; Andrist et al., 2012), management of the conversational floor Andrist et al. (2013, 2014), and efficiency in task collaboration Andrist et al. (2017). Finally, how the eyes, the head, and the body are configured affects the perception and outcomes of gaze cues Hietanen (1999); Andrist et al. (2012); Pejsa et al. (2015), highlighting the complexity of the role of gaze in social perception and the richness of the design space for gaze as an embodied cue in human-machine interaction.

\subsubsection{Facial Expressions}

Embodied agents have the opportunity to use a variety of facial features and expressions. Facial expressions can appear alongside other embodied cues or as isolated behaviors (Goffman, 1959); they strongly influence how an agent is perceived. While the complexity of faces makes them a rich and expressive channel of communication, this also makes the design of expressions challenging. Inappropriate expressions can result in strongly negative interaction performance, such as eliciting the Uncanny Valley phenomenon (Mori, 1970) that relates high-fidelity realism to the agent's features to perceived "creepiness". Furthermore, facial expressions that are incongruent with speech can confuse interaction partners (Mower et al., 2009).

Social smiles are particularly important in social interaction. They serve as salient back-channel cues that express understanding and agreement, improving conversational efficiency (Brunner, 1979) and perceived social competence of the robot (Argyle, 1988; 


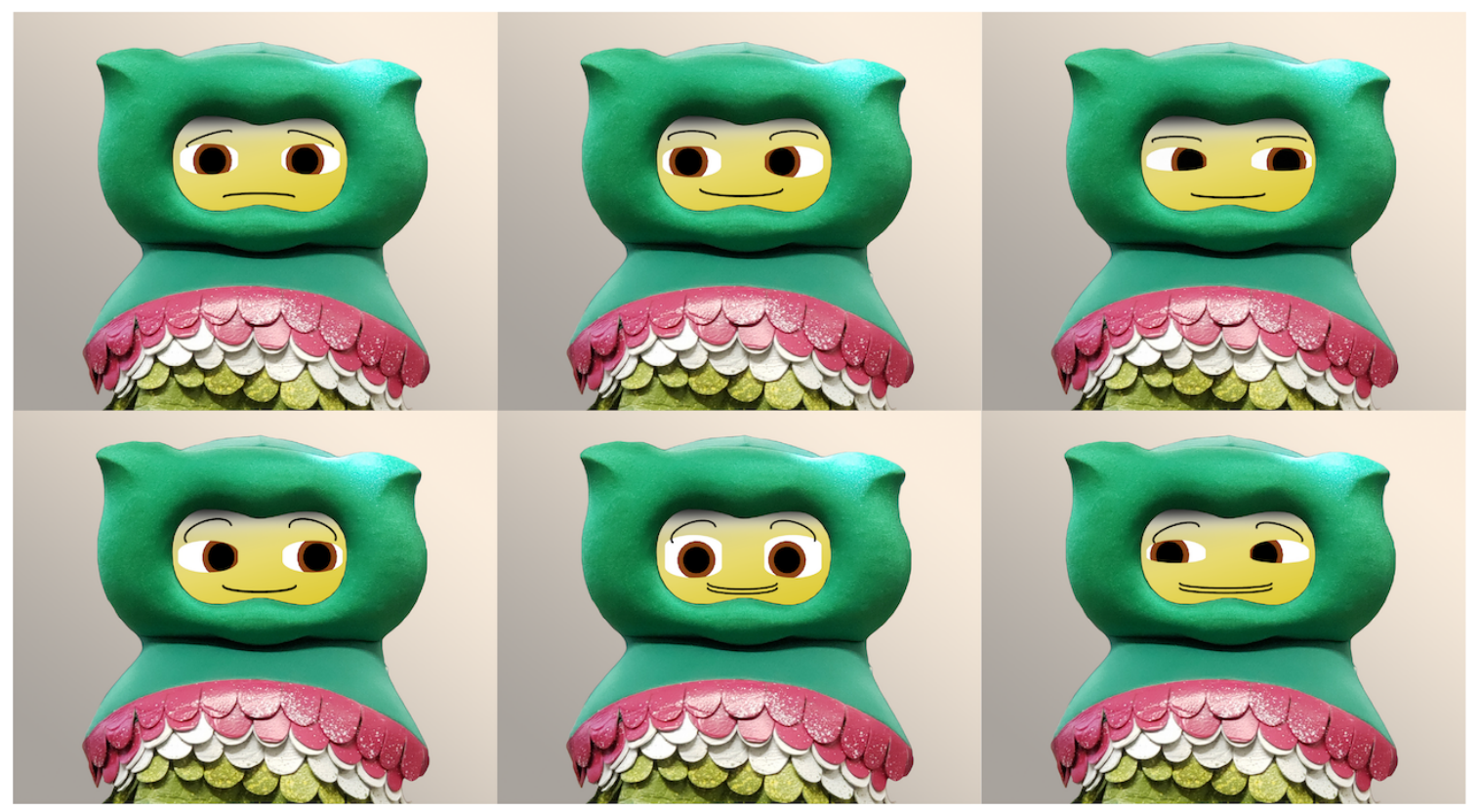

Figure 3.8: Different emotional expressions on the Spritebot platform inspired by expressions of human emotion.

Otta et al., 1994; Mutlu, 2011). Ill-timed or inappropriate smiles, however, have a strongly negative impact on interaction and can invoke the Uncanny Valley phenomenon.

Facial expressions influence the internal states of both the agent and the observer (Ekman and Friesen, 1975; Russell et al., 2003), and emotional expression and interpretation are associated with the activation of specific brain regions (Barrett, 2006b,a). This relationship provides an opportunity for informed design for interaction. Findings by Ekman and Friesen (1975) provided abstractions, such as "happy" and "sad," that serve as the most commonly used foundation for the design of facial expressions for anthropomorphic and zoomorphic robots. Such robots are often designed with faces that are more abstract than the rest of their bodies relative to their design metaphor in order to enable the effective use of facial expressions and to eliminate unnecessary complexity (Elaine S. Short and Matarić, 2017). Zoomorphic robot designs can also utilize human "facial action units," allowing designers to use human-like facial expressions on animal-like robots to express interpretable emotion (Elaine S. Short and Matarić, 2017; Kalegina et al., 2018). This technique effectively blends animal-like and human-like metaphors as the primary and secondary metaphors, respectively, as people are much less familiar with animal facial expressions. The design of the Spritebot platform is an example of this approach, blending feline and human metaphors in the design of the robot (Figure 3.8) (Elaine S. Short and Matarić, 2017). 


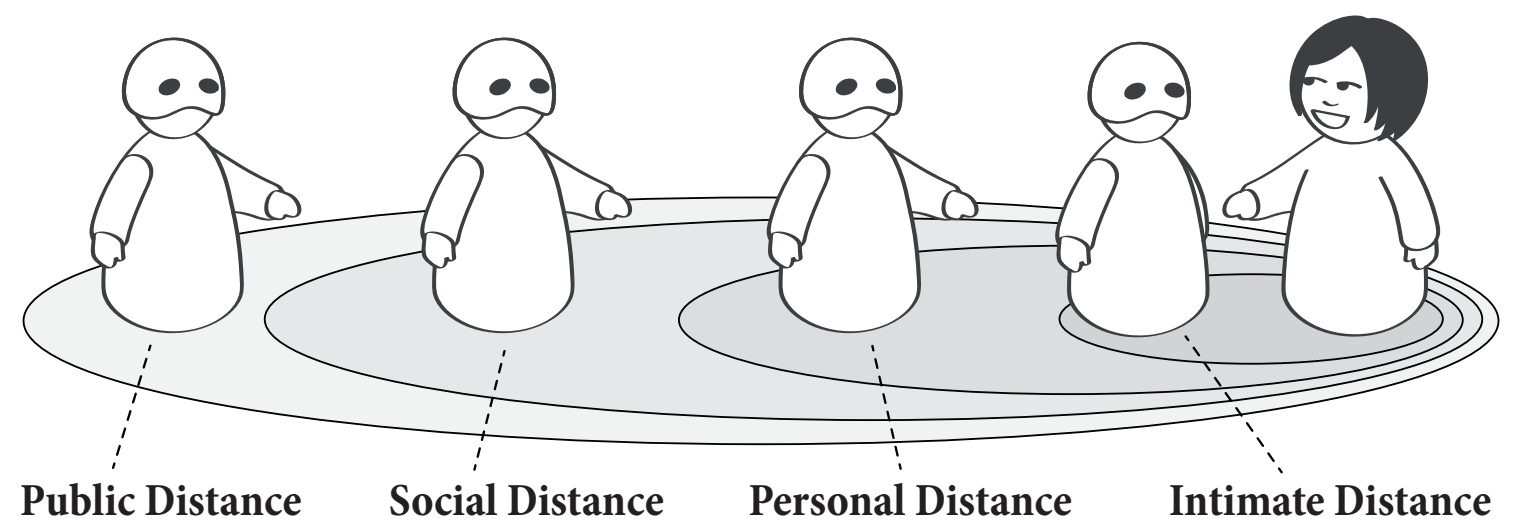

Figure 3.9: An illustration of proxemics zones suggested by Hall (1963).

\subsubsection{Proxemics}

The positioning of social agents in physical space relative to other interaction partners and objects also acts as a salient embodied cue in social interaction (Mead et al., 2013). The distance and orientation of interaction agents provide strong bidirectional signals for perception, intent, and attitude that are especially relevant for the design and implementation of mobile socially interactive robots (Mumm and Mutlu, 2011; Mead and Matarić, 2017). Research in human communication has long studied human proxemics, offering a number of models to predict how spatial behaviors affect interaction outcomes (Argyle and Dean, 1965; Hayduk and Mainprize, 1980). Work in human-robot interaction has provided experimental support for some of these models (Mumm and Mutlu, 2011) and has highlighted the importance of proxemic cues in the design of interactive behaviors for physically co-present robots (Takayama and Pantofaru, 2009; Walters et al., 2005). The design of these cues can drastically change how people perceive robots, e.g., as disruptive and threatening (Mutlu and Forlizzi, 2008) or as accepting and friendly (Mead and Matarić, 2016), underlining the need for careful consideration of proxemic behavior design.

\subsubsection{Social Touch}

Physical embodiment presents robots with the opportunity to physically interact with their environments, including their interaction partners. Social touch comprises non-functional touch-based interactions such as hand-holding or touches on the arm, shoulder, and face (Jones and Yarbrough, 1985; Gallace and Spence, 2010). In human-human interaction, social touch facilitates development, social connectivity, and emotional support, and helps communicators to establish and maintain engagement throughout interaction (Yohanan and MacLean, 2012; Jung et al., 2017; Gallace and Spence, 2010). When used according to 
human social norms, social touch cues can serve as salient signals for dominance, intimacy, immediacy, and trust (Mehrabian, 1972; Montagu and Matson, 1979; Burgoon, 1991).

\subsection{Summary}

This section introduced a characterization of the design space for socially interactive robots. Since design spaces have served as transformational tools in many design fields, our goal was to provide designers and researchers such a tool for embodied interactive agents. As socially interactive robots are complex systems, we analyzed their design aspects within three sub-systems chosen to parallel industrial design, interaction design, and animation.

The embodied cues discussed in this section make up the primary elements in the design space of interactive behaviors for socially interactive robots. When designed carefully and used within established social norms, such behaviors can enable rich, engaging, and effective interactions. The next section introduces the metrics used to discuss different facets of interaction performance, reviews results from the surveyed studies, and discusses the implications of those results on the design of the behaviors, embodiments, and interaction strategies of future socially interactive robots.

\section{Embodiment Study Outcomes and Design Implications}

This section introduces an overview of the various evaluation techniques used in the reviewed studies, grouping them into categories of measures and discussing their strengths, weaknesses, and domains of application. This categorization of evaluation techniques and the design space taxonomy from the previous section are then used to analyze results from the reviewed studies and present their design implications.

\subsection{Experimental Overview}

Our review of prior studies on embodiment in socially interactive robots covers a wide range of applications, user populations, and methodologies. We begin by introducing the set of experiments that we evaluated and discuss the overall landscape of embodiment studies at the time of this review. Of the 65 experiments in our review that compared a physically embodied or strongly embodied agent to a comparable virtually embodied or weakly embodied agent, 50 experiments compared two types of embodiments, 11 experiments compared three types of embodiments, and 4 experiments compared more than three different types of agent embodiment. Of the 65 total experiments, 17 involved more than 60 participants, 24 involved between 30 and 60 participants, and 24 involved fewer than 30 participants (Table A.4). In Figure 4.1, the reviewed experiments are mapped on the task circumplex (Mcgrath, 


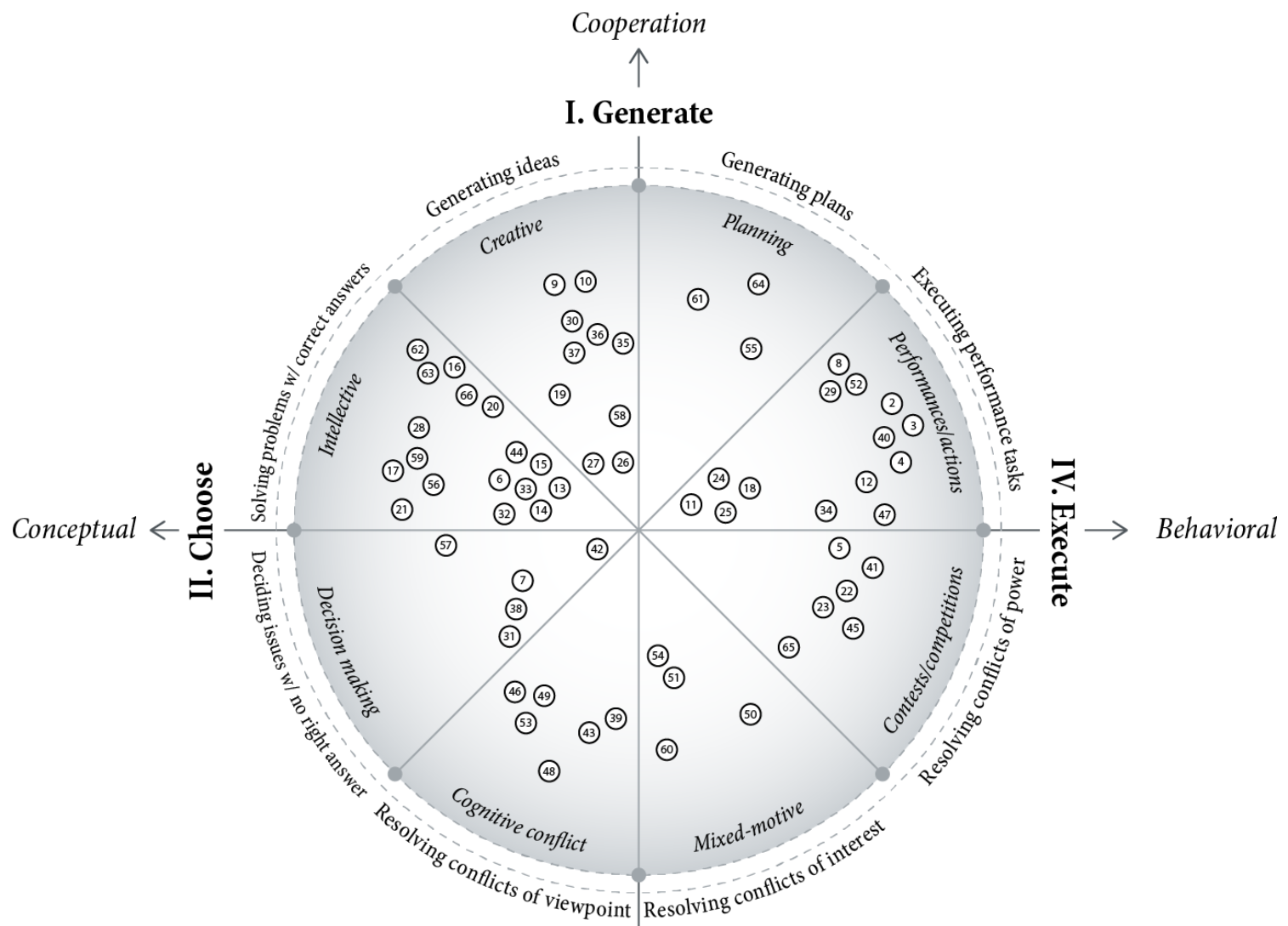

\section{Negotiate}

$\downarrow$

Conflict

Figure 4.1: Studies included in our review, overlaid on McGrath's (Mcgrath, 1995) task circumplex. The distance from the center indicates social role: inward = subordinate, outward $=$ superior.

1995). The social role of the robot is represented by the distance from the center: the closer to the center, the more subordinate and the further from the center, the more superior.

\subsection{Interaction Outcomes and Measures}

As discussed in earlier sections, studies of embodiment in the humanities and social sciences predate research on the embodiment of robots and artificial agents. Some of the techniques used by researchers in those fields have been adopted into robotics-related research. Validated observational instruments, such as the POMS survey (Garau et al., 2005), the semanticdifferential scale (Snider and Osgood, 1969), and selected quantitative techniques from 
Mosteller et al. (1954), have been implemented in a number of studies related to embodiment in robotics (Goodrich and Schultz, 2007). These measurement tools are especially valuable for evaluating the more subjective results of experiments involving socially interactive robots and can provide valuable insight into the state of the embodiment hypothesis. For instance, Lee (2004) provided empirical evidence for the mediating role of presence in people's social responses to synthesized voices; Experiment 1 described by Lee et al. (2006) showed that people evaluated both the physically embodied agent and the interaction they had with it more positively and characterized physical embodiment as "an effective tool to increase the social presence of an object."

In previous sections, we described the relevant design elements of socially interactive agents and discussed how they can affect the quality of user interaction with robot systems across different tasks in various social contexts. A remaining challenge is how interaction quality is defined and measured. Although prior research on embodiment captured a large number of dimensions of interaction quality, we classify measures that are used to capture these dimensions into two categories: behavioral (or observed) measures and subjective (or self-reported) measures (Goodrich and Schultz, 2007). Figure 4.2 illustrates these categories. Most studies use a combination of the two and specific measures for each reviewed experiment can be found in Table A.5). In the following subsections, we discuss the different measures used in the reviewed studies to provide an overview of how embodiment studies assess interaction quality.

\subsubsection{Self-Reported Metrics}

Self-reported measures are metrics of interaction quality collected from study participants in the form of responses to structured, semi-structured, and open-ended survey instruments. These measures give researchers the ability to capture interaction quality as perceived by participants and are especially helpful in differentiating the various facets of user experience with the robot, such as the participant's perceptions of the robot's intelligence, how much trust was established between the user and the robot, and how enjoyable participants found the interaction to be. Example self-reported measures used in prior work include open-ended interviews (Ju and Sirkin, 2010; Takeuchi et al., 2006) and questionnaires designed to capture various dimensions of interaction quality, including social attraction (McCroskey and McCain, 1974), perceived intelligence (Krogsager et al., 2014), and story appreciation (Costa et al., 2016). Table 4.1 provides a full list of the self-report measures used in the reviewed studies.

A key consideration in the use of self-reported measures is the type of data to be collected from participants. Semi-structured and open-ended interview methods provide rich, qualitative data, while questionnaire-based survey instruments, structured using rating scales such as the Likert scale (Allen and Seaman, 2007), provide quantitative measurements 
Self-report: Subjective evaluations

(e.g., rapport, liking, trust, attractiveness, affiliation, perceived intelligence, perceived competence)
Observed: Interaction

(e.g., turn-taking frequency, amount of mutual gaze, number of interruptions, overlapping speech, mutual proximity)

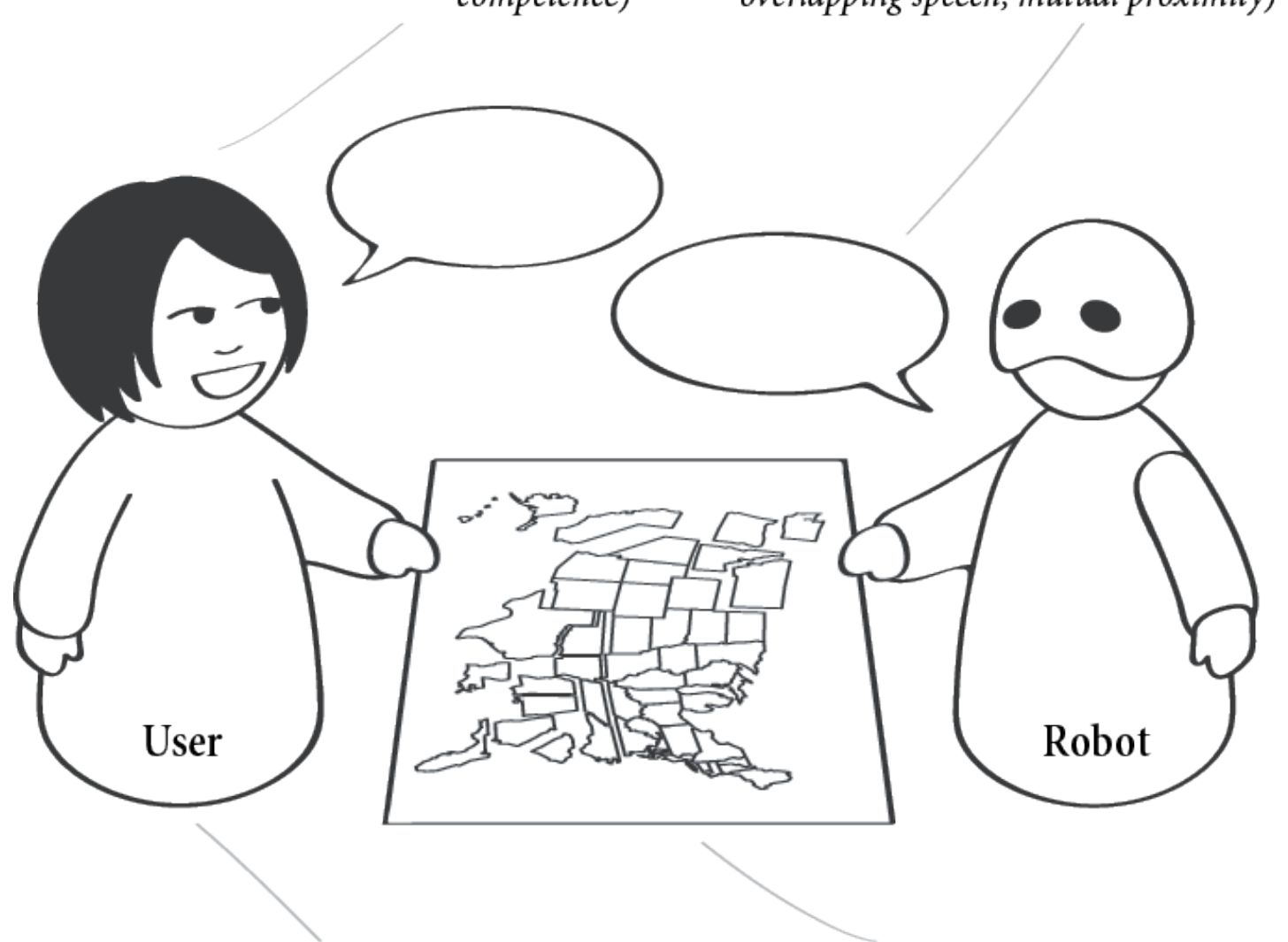

Observed: Individual behavior

(e.g., gaze fixation length, amount of smiling, number of deictic references, speech rate, speaking volume)
Observed: Task performance (e.g., information recall, number of correct identifications, time to task completion, error rate)

Figure 4.2: Measures of human experience with socially interactive robots.

of specific variables. For guidelines on designing interview questions and questionnaire-based measures, see Louise Barriball and While (1994) and Hinkin (1998), respectively. 


\subsubsection{Observed Metrics}

Observed measures capture user task-related actions, physical behaviors, and physiological responses that can be observed by human experimenters or measured using sensing instruments. These measures can be evaluated in real time or post hoc and can be viewed from three perspectives: (1) individual behavior, (2) interaction, and (3) task performance. The following paragraphs describe each perspective.

Individual behavior involves observed measures of a user's behavioral, task, or physiological state over a period or at specific points in the interaction. Measures of user behavior include body motion (Noldus, 1991; Chang et al., 2005), body pose (Brooks et al., 2012), gaze behavior (Mutlu et al., 2009, 2012), facial expressions (Costa et al., 2016), and linguistic verbosity (Fischer et al., 2012). Table 4.2 lists measures of individual behavior used in the reviewed studies, the methods with which they were labeled or analyzed, and studies that included these measures.

Interaction measures capture interactive phenomena that emerge through interaction among interaction partners. For example, while directed gaze toward an object of interest in the environment serves as a measure of individual behavior, mutual gaze emerges from two parties establishing and maintaining eye contact and serves as an interaction measure. Table 4.3 lists the interaction measures we observed in reviewed experiments along with the analysis or labeling methods used and some sample experiments in which these measures were used.

Measurements of task performance capture the effectiveness of the user or the group in performing the primary task of the interaction, such as effective learning in an educational interaction (Huang and Mutlu, 2013) or speed of assembly by a human-robot manufacturing team (Pearce et al., 2018). Most applications of socially interactive robots aim to support at least one quantifiable, task-oriented measure focused on the tasks in the given interaction context. In the majority of the reviewed experiments, researchers were observing interactions with defined task goals, such as performance in games (Fridin and Belokopytov, 2014), negotiation (Bartneck, 2003), or imitation (Robins et al., 2006). These goals include explicit metrics of performance that can be used as a grounded measure of user behavior. Because task performance is inherently a contextual measure that is commonly specific to individual experiments, these metrics are highly varied. Designing task performance measures for a given study is best informed by previous work (Appendix A.1) in similar task categories.

As many existing studies in socially interactive robots explore new domains, applications and interaction scenarios, the research literature still lacks established and validated selfreported or observed measures. Additionally, the majority of studies to date involve shortterm interactions, and systems lack the ability to capture measurements over long periods. As speech recognition, language understanding, affect recognition, activity understanding, 

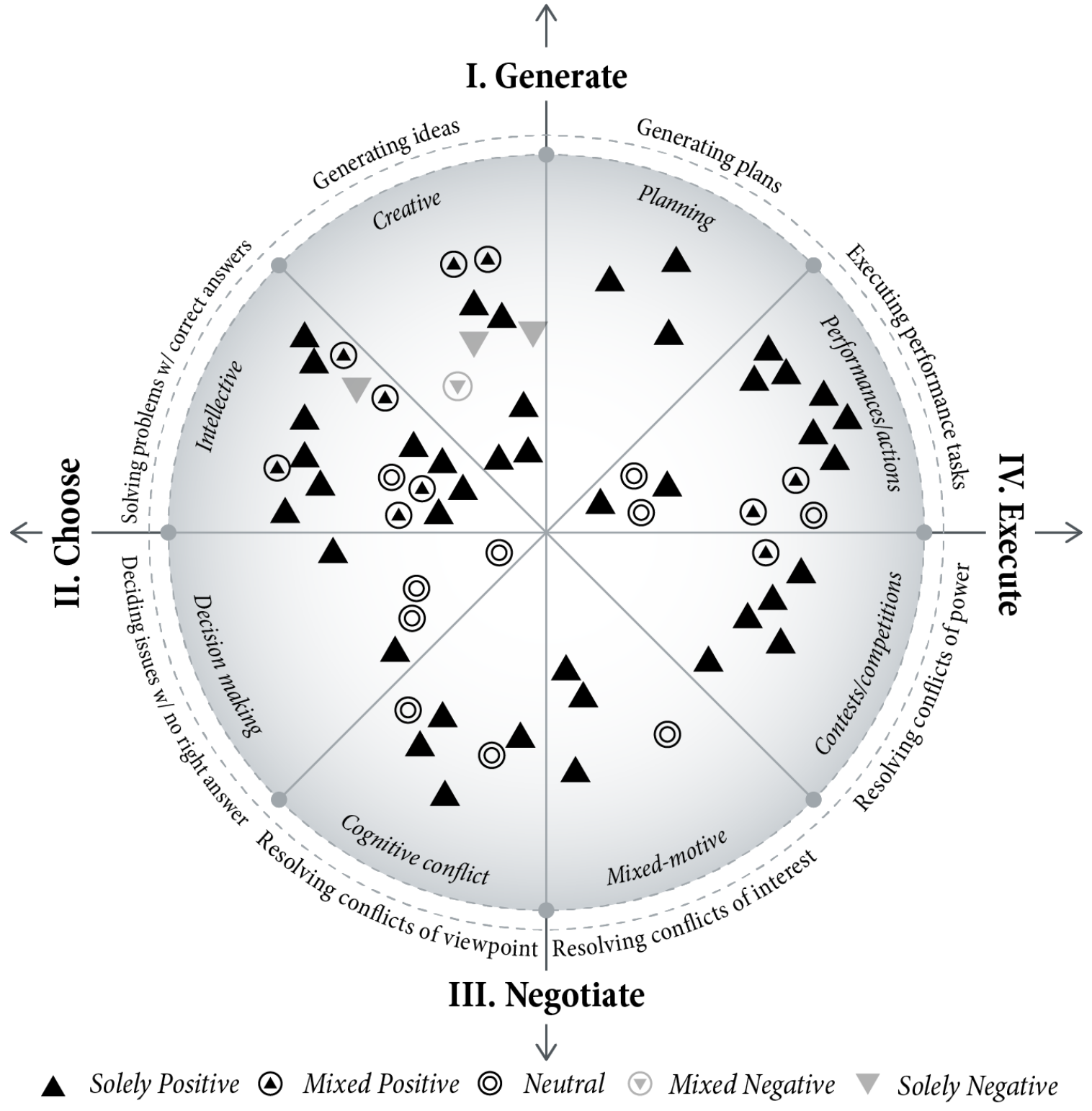

Figure 4.3: Combined results for all reviewed studies.

and other relevant technologies improve and systems become increasingly robust, automated methods for behavior measurement over long periods will become the norm. 


\subsection{Effects of Embodiment on Interaction Outcomes}

The reviewed studies all seek to understand the effects of embodiment on human interaction with socially interactive robots in order to develop design guidelines for future computer and robot systems. In this section, we summarize the results of the reviewed studies with respect to this central research question.

Based on the observed and self-reported measures taken, the reviewed experiment results can be grouped into two types: differences in the perception of the agent and differences in task performance (Table A.3). By combining these two measures, all experiment results can be classified into five categories relative to the embodiment hypothesis: (1) solely positive (63.1\%), (2) mixed positive (15.4\%), (3) neutral (15.4\%), (4) mixed negative $(1.5 \%)$, and (5) solely negative $(4.6 \%)$. Over all reviewed experiments, the results are strongly positive in support of physical embodiment, with $63.1 \%$ of combined results showing improvements in interaction and performance and $6.1 \%$ showing negative results (Figure 4.3).

The two measures, task performance and agent perception, are not fully separable, so analyzing both categories of measures provide a fuller and more nuanced understanding of interaction outcomes. For instance, Segura et al. (2012) studied participants' preferences when given the option to interact with a physically-embodied robot companion or with a virtual representation of that robot. They reported that, although participants found the robot "less annoying" and explicitly chose to interact with it more than the virtual agent, their ratings of the different embodiments did not reflect these observed functional preferences. The authors of that work deduced that choosing between an embodied or simulated agent was very task-specific. For tasks that involve a significant amount of information transmission but relatively little social rapport (e.g., information kiosks), and for tasks that require users to reveal personal information, disembodied agents should suffice. However, for tasks that are relationship-oriented (e.g., a home companion), social engagement is important for maintaining rapport, and physical embodiment is beneficial for increasing social presence, and in turn, engagement and rapport.

In the following subsections, the results of the reviewed studies are examined across the agent performance and perception categories, and analyzed relative to the current state of the embodiment hypothesis in socially interactive robots.

\subsubsection{Differences in the Perception of Agent}

The primary method for evaluating the social performance of artificial agents measures users' perceptions of those agents, and changes in those perceptions as a result of interactions. The affordances gained by the design of a robot, the behaviors of that robot, and demonstrated competence are all key components of the resulting user perceptions. Researchers have aimed to study specific features such as attachment, comfort, loneliness, and general attitudes 


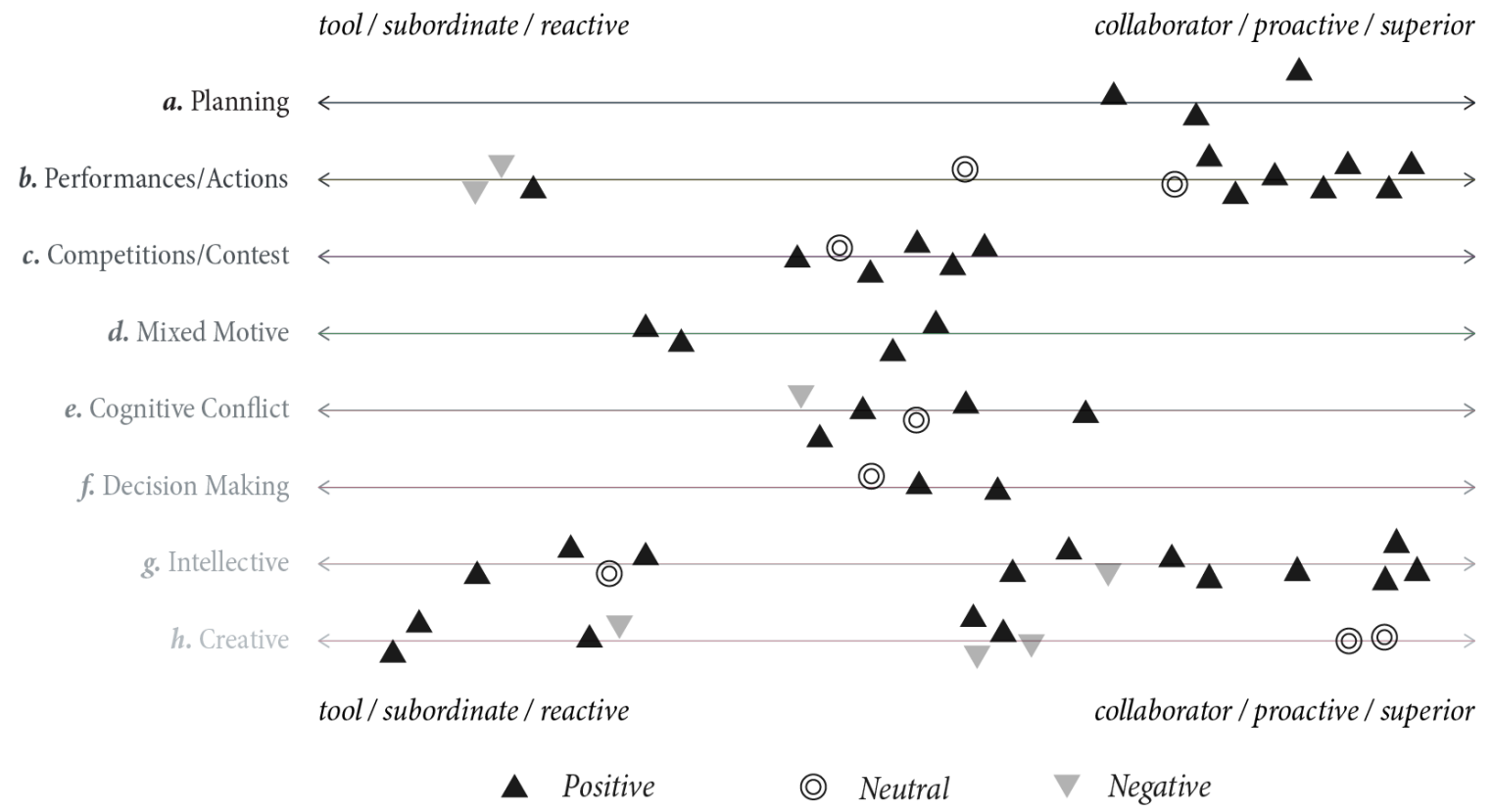

Figure 4.4: Survey results for interaction performance differences between physically embodied and otherwise-embodied agents.

towards robots (Bartneck et al., 2009), in isolation from other factors such as novelty effects and prior task experience.

In our review, 57 of the 65 studies measured differences in the perception of the artificial agent using a variety of observational instruments. The majority of the experiments used a combination of observed and self-reported measures. The results reporting on agent perception are found in Figure 4.4 on the task circumplex. Each point represents a singular experiment, and its color represents the finding; green represents physical agents outperforming their non-physical counterparts; yellow represents a neutral result; and red represents virtual or non-embodied agents outperforming the physical agents.

Of the 57 experiments, $43(75.4 \%)$ showed that using a physically embodied agent is superior in improving user perceptions of the agent. Every task category had a majority of results favoring physical embodiment; four of eight task categories had positive or neutral results. Our review provides support for the embodiment hypothesis in the context of agent perception based on the current state findings in the field.

While 43 experiments had results supporting the embodiment hypothesis, 7 experiments presented negative results, and another 7 experiments presented neutral results. Most of the neutral results (shown in yellow in Figure 4.4) have task and role classifications comparable to experiments with supportive results (shown in green), suggesting that these 
neutral results may stem from other facets of experimental design and may not indicate the impact of embodiment. Because of the subjective nature of measuring agent perceptions, we postulate that the lack of statistical significance in these experiments could be due to high data variance.

The two experiments with neutral results not completely surrounded by positive results are in the creative task category and are both the most "superior" agents used in their respective task categories. This pattern, along with the three negative results for the most "peer-like" agents in the same task category, is a strong indicator for the importance of social roles in certain types of tasks. We speculate that creative tasks may be a task category particularly sensitive to social roles, as the creative process involves socially complex interactions.

The seven negative results form clusters within their respective task categories. As Figure 4.4 shows the results of studies as a function of task type and social role, the clustering along the dimension of social roles within each type of task provides further evidence for the importance of appropriately designing social roles in the perception of artificial agents for different types of tasks.

Perception of the robot agent is affected by three factors: (1) the design of the robot (design metaphor and abstraction level), (2) the behaviors of the robot, and (3) the perceived social role of the robot. Depending on the type of interaction or task and the duration of the interaction, the relative importance of these factors can vary. The design of the robot scaffolds interactions by setting expectations about the robot, including signaling its physical and cognitive capabilities and its ability to follow norms of human social behavior. For example, a humanoid robot with a dynamic mouth is more likely to be expected to have conversational capabilities than a cat-like robot with a molded mouth. A robot with realistic-appearing arms is expected to be able to perform both gesture and manipulation tasks while a robot with stylized arms may only be expected to perform simple or high-level gestures.

People are primed by the perceived social role of the robot. For example, users may hear out a subordinate robot but not comply with it (Waldron, 1991). Failure by a superior agent in generative tasks, or tasks that involve collaboration between the robot and person to co-create ideas or narratives, can be interpreted as incompetence, while failure in negotiation tasks can be interpreted as potentially manipulative (Caldwell and O'Reilly III, 1982). When suggestions from subordinate agents fail, people may be more likely to take the blame for the failure of the suggestion - it was not the agent's incompetence that caused the failure, because the person, as the superior agent, should have known not to take that advice.

The perceived social roles and expected behaviors of robots are not static; through demonstration of their functional and social abilities, robots can show to their human interaction partners what they are capable of. The length of the interaction is particularly 
important factor that shapes the effects of robot design and behavior on agent perception. For example, in short-term interactions, the affordances gained by the "first impressions" of the robot, typically stemming from the design of its embodiment, play particularly important roles in user perceptions of that robot. In longer interactions, users are given more time to observe the behavior and demonstrated capabilities of the robot and can adjust their first impressions accordingly. For instance, if a robot is perceived to have manipulation capabilities based on its embodiment, and it fails at manipulating objects that are too heavy or too large, the perception of the robot' physical capabilities will be impacted negatively, and the expectations of the robot will become more realistic. As people's expectations are calibrated by the robot's demonstrated behavior, the affordances and impressions first gained from the embodiment become less important (Segura et al., 2012).

The robot's task is especially important in the context of perceived social roles. In the reviewed experiments, there were some discrepancies between intended and perceived social roles and, in some cases, the social roles were themselves the experimental variables (Elaine S. Short and Matarić, 2017). The tasks provided contexts in which social roles could be evaluated.

Overall, the experiments we reviewed provide strong support for the value of physical embodiment in perceived social competence, measured by people's perceptions of the artificial agents. Furthermore, our interpretations of the few negative results highlight the need for proper embodiment design. By measuring human perceptions of artificial agents, researchers aim to study their social capabilities that serve as fundamental context for task performance, as discussed in the next section.

\subsubsection{Differences in Task Performance}

Agent-agent interactions typically aim to accomplish a set of shared goals. Those goals can be abstract, such as "have a discussion" (Powers et al., 2007), or explicit, such as "move all books from current locations to goal locations" (Bainbridge et al., 2011). Within the shared goals, each individual has their own goals that may represent conflict (bottom half of the task circumplex) or cooperation (top half of the task circumplex). Compared to measurement tools used for agent perception and social performance, metrics of task performance involve observation-based, objective measures, such as measuring response time (Bainbridge et al., 2011), the number of moves in a puzzle (Hoffmann and Krämer, 2013), or the compliance rate (Komatsu, 2010). Pairing task performance and agent-perception measures can provide a more complete understanding of interaction outcomes. Because socially interactive robots are usually designed to accomplish or support a task, even if it is as general as engaging a user for a set amount of time, task performance measures can be seen as the "end result" of the robot's performance. 


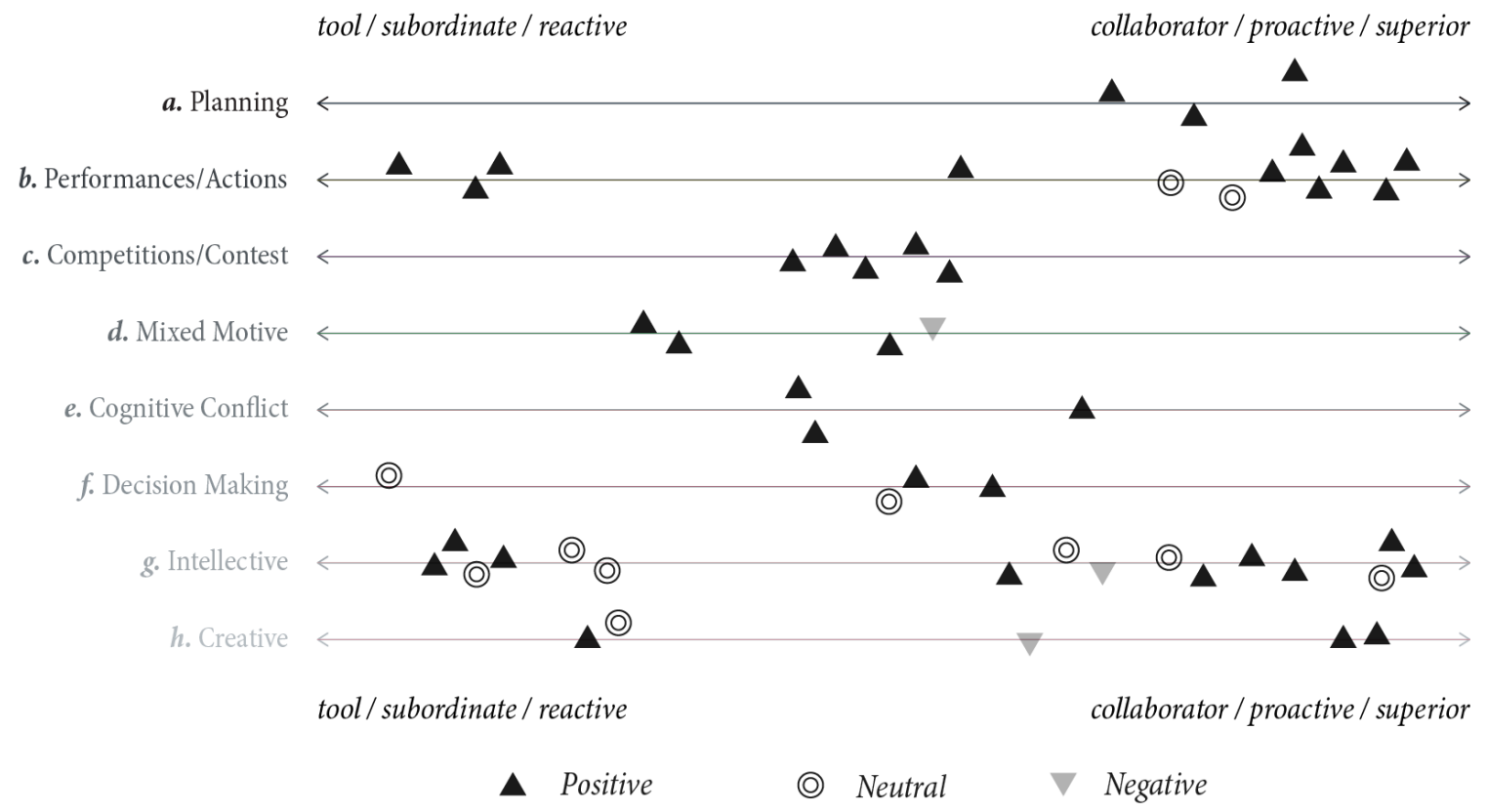

Figure 4.5: Survey results for task performance differences between physically embodied and otherwiseembodied agents.

Of the 65 studies we reviewed, 57 used defined metrics for task performance; a majority showed significant improvements in user performance when collaborating with physically embodied robots. These task performance results, plotted over the task categories and social roles, can be seen in Figure 4.5. Of the 57 experiments, 37 presented positive results $(71.15 \%)$ for having a physically embodied robot over virtual or disembodied agent, leading to the conclusion that physical embodiment is beneficial for improving the social performance of artificial agents as well as task performance of human users interacting with those agents. For instance, Jung and Lee (2004) found that, when interacting with the eMuu robot, participants scored higher on the negotiation task than when they were interacting with a virtual rendering of the robot. Bainbridge et al. (2011) presented results for a book-moving task in which the artificial agents requested unusual behaviors such as throwing books into the trash; the results showed higher compliance rate when requests came from a physical robot than when they came from a virtual agent. In learning tasks, Jost et al. (2012a) also showed children to be significantly more motivated by a physical robot when playing a cognitive-simulation game.

Although the majority of the reviewed experiments demonstrated task performance improvements, 3 did not, and 12 had neutral results. In comparing non-positive results in agent perception and task performance, we note that their overall total numbers are 
similar (14 and 15, respectively), but there are almost twice as many neutral results in task-performance measures than in agent-perception measures. The neutral results fall into four of the eight task categories and are clustered by social role within those categories. For instance, in performance and actions, experiments show improved task performance when the embodied robot is playing either a superior or subordinate role, while all studies with neutral artificial agents have neutral results. Fasola and Mataric (2013) used a humanoid robot, Bandit, to lead "chair aerobics" exercises with older adults in the US, and Nomura and Sasa (2009) used a robot to give directions to adults in Japan for sorting objects into boxes. The robots played similar roles and, due to the nature of these two tasks (i.e., "generation" and "execution"), the peer-like role of the robots may not have inspired as much confidence in the robots' intellectual contributions as superior robots would, nor did it cause users to feel the need to support the robot as subordinate agents would.

The three negative results are all in different task categories and, within those categories, are located near the largest cluster of neutral results. This clustering may be an indicator of social roles that are less effective than others within a given type of task, making designing a robot for that application in that role more difficult.

Overall, the results of the reviewed experiments provide strong support for physical embodiment in task performance with socially interactive robots. Compared to results for agent perception, the results for task performance had fewer negative results and more neutral results. We speculate that the effects of embodiment on user task performance, which can be independent of the robot, are not large enough to reveal a statistically discernible difference, while its effects on agent perceptions, which involve evaluations directed toward the robot, may be stronger. This interpretation presents future challenges for embodiment design, e.g., designing more salient embodiments, and highlights the nuanced and complex effects of embodiment on human interaction with socially interactive robots. The next section draws on the insights from our findings and presents design recommendations and considerations for future studies.

\section{Recommendations for Future Embodiment Studies}

A wide range of behavioral research paradigms are available for studying the role of embodiment in human interaction with socially interactive robots; Mcgrath (1995) provides a comprehensive discussion. This chapter outlines common research paradigms, study designs, independent variables, and measurements for studies of embodiment in socially interactive robotics, concluding with a discussion of limitations and open questions for such studies. We do not attempt a comprehensive survey and refer the reader to the literature on research methods for studying human interaction with technology (e.g., Lazar et al. (2017) 
and Olson and Kellogg (2014)) and on behavioral research methods in general (e.g., Cozby and Bates (2017) and Price et al. (2017)).

\section{$5.1 \quad$ Research Paradigms}

Research studies of embodiment in socially interactive robots to date have primarily involved controlled laboratory studies. As socially interactive robots become more pervasive, future studies will need to consider methodological fit (Edmondson and McManus, 2007) and draw on a richer set of choices under both laboratory and in situ studies.

Laboratory studies allow for a higher level of control over the variables in the phenomena being studied, at the cost of ecological validity, the extent to which findings in the laboratory can be generalized to real-world situations. In situ studies identify representative settings, i.e., the "field," of the target environment for the design of the system, introduce the system to these settings, and enable the study of human interaction with the system using comparative or naturalistic research paradigms. For example, an in situ study of an educational robot designed to improve student attention to instruction may be conducted in a real-world classroom and seek to confirm that the attentional benefits of the design can be obtained in the complex and dynamic setting of a classroom.

In situ studies are carried out in the natural setting in which a system is deployed or the target setting for which a system is designed. Naturalistic studies involve no experimental control and follow ethnographic and other field methods to capture the natural and emergent ways in which humans interact with robots. For example, Mutlu and Forlizzi (2008) conducted a study of how workers at a hospital interacted with a delivery robot, utilizing ethnographic observations and interviews to capture behavior as well as subjective perceptions of the robot.

Comparative in situ studies, or "field experiments," in contrast, involve introducing robot into a target setting, manipulating aspects of the robot's design, and using qualitative and quantitative methods to understand how these manipulations affect human interaction with the system. For example, a field study conducted by Hayashi et al. (2007) introduced two socially interactive robots into a train station, varied how active and social the robots acted, and studied commuters' interactions with and perceptions of the robots. Such studies involve some control of the system's behavior or the environment (e.g., where or how the system is introduced to the setting) while allowing all other variables to vary naturally.

\section{$5.2 \quad$ Study Designs}

The design of a study is determined by how much control is desired, whether independent variables can be manipulated, and how many variables are considered. Studies in social 
human-robot interactions involve four key study designs: true experiments, quasi experiments, system-level evaluations, and naturalistic studies.

Both quasi and true experiments seek to establish causal relationships between design variables and outcomes of interactions with robots, although they differ in whether or not experimental conditions are randomly assigned to the population of interest and thus in the conclusiveness of the causal relationships identified by the study. Additionally, true experiments are most commonly used in laboratory studies, while quasi-experimental designs are most common in in situ studies.

In true experiments, participants sampled from the population are randomly assigned to study conditions that correspond to different levels of an independent variable. For example, a study on the effects of physical proximity between the robot and its user may establish "close" and "far" distances at which the robot will interact with its user, randomly assign members of its study population to these levels, and use inferential statistics to determine whether the amount of distance had a significant effect on participant behaviors or perceptions of the robot.

Quasi experiments, on the other hand, are used in situations where random assignment is not possible, and studies compare matched groups or make pre-/post- comparisons. For example, a study that compares the use of a robot across two senior living facilities or a study that compares social interaction among members of a facility before and after the introduction of a robot. While quasi-experimental designs can allow the exploration of settings or interactions that are otherwise impossible to study and can offer valuable insights, their findings are less conclusive than those obtained in true experiments.

Both true experimental and quasi-experimental study designs have inherent limitations when used in the context of research into socially interactive robotics. First, they offer insight into relationships between a small number of design variables in isolation and lack the ability to conveniently study large design spaces that robotic systems involve. Second, they usually show that manipulations of variables significantly affect interaction outcomes but do not provide an understanding of the extent of these effects, limiting the ability to make fine-grained design decisions to meet the specific demands of a robot product. System-level study designs seek to address these limitations by simultaneously modeling the predictive relationships between a large number of design variables and interaction outcomes. Research in socially interactive robotics has utilized two variations of this approach. The first variation involves asking users to interact with a socially interactive robot in the way it is intended and ad hoc modeling of predictive relationships between design variables and interaction outcomes. For example, Peltason et al. (2012) asked participants to perform an object-learning task with a robot and modeled the predictive relationships between design variables such as how many utterances the robot spoke per minute, as they are naturally utilized in the interaction, and interaction outcomes such as perceived ease of use of the robot using multivariate regression techniques. The second approach utilizes the 
same statistical modeling tools, but instead of modeling variable-outcome relationships, it explicitly manipulated multiple design variables simultaneously within their possible ranges. Huang and Mutlu (2014) demonstrated the use of this method in a study on the design space of arm gestures for a socially interactive robot; they manipulated the frequency of each type of arm gesture and modeled how well the use of each arm gesture predicted interaction outcomes. They found, for example, that the use of pointing gestures by the robot significantly predicted information recall in participants. Furthermore, they found that each standard deviation increase in the use of this type of gesture increased information recall by 0.123 and 0.623 standard deviations for females and males, respectively. This example illustrates the power of system-level study designs in gaining a fine-grained understanding of variable-outcome relationships, which can enable fine-grained decisions in the design of the robot system.

Finally, research into embodiment in socially interactive robotics also benefits from naturalistic studies that involve minimal levels of control. These studies utilize methods from ethnography, the systematic study of people, settings, organizations, and cultures based on observational data, and other forms of qualitative empirical research, including fly-on-the-wall observations, participant observation, interviews, and system-log data. Data obtained using these methods are analyzed rigorously using qualitative methods. Such studies seek to utilize the rich data obtained from the setting coupled with rigorous analysis to arrive at a deeper understanding of the use of the robot system in the study setting.

\subsection{Independent Variables}

A common characteristic of all studies of embodiment in socially interactive robotics is inquiry into the effects of system-level properties of embodiment on the interaction, including the high- and low-level variables that make up the design of the robot. In lab and in situ studies that involve experimental control determine these properties and variables a priori. Naturalistic studies, on the other hand, explore those effects in an unstructured fashion, although they can also seek to describe studied phenomena without drawing any conclusions about them. In experimental design, those properties are called independent variables and refer to factors in the study that are explicitly manipulated, such as the height of a robot, or measured, such as the age of a participant. The interaction outcomes, such as how approachable participants find the robot, are referred to as dependent variables. The goal of the study is to understand how the independent variables affect the dependent ones.

Embodiment studies consider system-level independent variables that include whether the system is physically embodied, virtually embodied, or disembodied (such as a speechbased interface). Other independent variables include high-level properties of the design of the robot system, such as the metaphor followed in the design of the system. For instance, 
Hinds et al. (2004) compared robots designed to follow human and machine metaphors to understand the effect of human-likeness of the robot on the attributions that human collaborators made to the robot. Finally, independent variables also include low-level design variables, such as the distance a robot maintains between itself and its user, the amount of eye-contact the robot establishes with its user, and the overall height of the robot system. These low-level properties can be variables that vary on a continuous scale, such as height, distance, or frequency, or among a discrete set of options, such as the color of the robot's lips, as manipulated by Powers and Kiesler (2006) and found to affect participant perceptions of the robot.

\subsection{Measurements}

Understanding variable-outcome relationships in studies of embodiment requires the appropriate definition of dependent variables that are expected to be affected by independent variables of interest and the appropriate measurement of those variables. These measurements, as we previously discussed, can be categorized into observed and self-reported.

Observed variables include physiological reactions, behaviors, interactions, and task actions that can be reliably recognized, described, and quantified by third-party human coders, sensors, and recognition algorithms. Specifically, observed task actions of participants can be translated into standardized task-performance measures, also called "objective" measurements, that can be used in quantitative analyses. For example, in a task in which participants collaborate with a socially interactive robot to sort toy blocks, the number of blocks sorted by the participant or the team within a period of time can be calculated from observed task actions and can serve as a measurement of task performance. Similarly, observed participant behaviors, such as gaze shifts, gestures, and speech, can be coded into behavioral variables that can serve as indicators of high-level cognitive processes. For example, the targets and timings of the gaze shifts of participants can be translated into measurements of gaze fixation toward particular types of targets, which can signal the amount of attention paid toward these targets. Measurements from observed behaviors can be automatically extracted using technology, including sensors and recognition algorithms, such as eye-tracking technology that can automatically translate gaze behaviors into gazefixation measurements. The use of overt physiological reactions such as body temperature as measurements, however, require the use of technology. Finally, the interdependent behaviors of multiple agents, humans and/or robots, as they unfold over time can be translated into measurements that indicate the fluency of the interaction. Examples of interaction measurements extracted from observed behaviors include rate of turn taking in conversation, the amount of mutual gaze between a robot and its user, and the mutual physical distance maintained between parties in an interaction. 
While physiological responses, behaviors, and task actions can be observed and then reliably translated into quantitative measures, participant attitudes, perceptions, and experiences are only accessible through the use of self-report measurements. Common methods to obtain self-report measures include the use of validated survey instruments such as multi-item questionnaires and translating transcriptions of interview data into quantitative metrics. The development of validated survey instruments that are appropriate for socially interactive robotics research is still in its infancy, and thus research to date has adapted validated scales from other fields and domains, including social psychology, interpersonal communication, and human factors, to study user attitudes toward and perceptions and experiences with socially interaction robots or gauge the cognitive, affective, and attentional states of participants. For example, the NASA Task Load Index (TLX) (Hart, 2006) is commonly used to measure user task load when interacting or collaborating with a robot. Researchers have also coded interview transcripts to extract subjective measures such as the frequency of the use of words with positive or negative valance.

Measurements in naturalistic studies primarily use qualitative data; other study designs can supplement quantitative measurements with qualitative data. Qualitative data most commonly take the form of rich narrative descriptions of studied settings and transcriptions of interviews conducted with study participants. While technology such as audio- and video-recording can be used to conveniently capture observations and interviews, commonly used methods for qualitative data analysis, such as content analysis (Krippendorff, 2004) and Grounded Theory (Strauss and Corbin, 1997), require textual data.

The choice of measurement can be guided by specific design goals or hypotheses. For example, a study investigating the extent to which an instructional robot improves student learning may use observed task-performance measures that indicate learning effects, such as recall of instructional material or ability to correctly apply it to a given problem. However, the scenarios and settings are usually complex, requiring researchers to use triangulationthe use of two or more measures, methods, or approaches to assess a single relationship (Rothbauer et al., 2008) - in order to improve the validity, confidence, and conclusiveness of findings. For example, the study on the learning benefits of the instructional robot may find significant learning effects, although this benefit may come at the expense of positive student experience. Triangulation by simultaneously measuring cognitive and affective learning would enable the researcher to gain a more comprehensive understanding of interaction outcomes and reveal potential tradeoffs.

\subsection{Hypotheses}

Rigorous application of many of the research paradigms described above require the development and testing of a set of hypotheses on the relationships between design variables 
and interaction outcomes. Naturalistic studies are generally incompatible with hypothesistesting. Controlled laboratory or in situ studies, on the other hand, involve a priori consideration of independent and dependent variables and thus provide necessary elements to construct testable hypotheses.

A key consideration in hypothesis development is the basis of the prediction made by the researcher. In the context of socially interactive robotics research, hypotheses are constructed by drawing from three key sources: prior research, pilot data, and design goals. Prior research may suggest understudied but plausible variable-outcome relationships or offer preliminary findings that require more conclusive evidence. Such preliminary evidence can also be obtained from pilot studies. In socially interactive robotics, design goals can also inform the development of hypotheses, as the justifications for the design choices for a system can provide sufficient bases for an expected outcome.

\subsection{Limitations and Open Issues}

Current paradigms and practices in studies of human interaction with socially interactive robots have a number of limitations for consideration by future theoretical and methodological advancements. A fundamental limitation is the integrated nature of robotic systems that only allows isolating specific design variables in the context of the holistic design of the system. This introduces two problems. First, and most fundamentally, robotics is challenging because of a large number of factors including uncertainties and limitations of perception and action and difficulties of repeatable behavior, as well as the challenges of robust behavior and finally the costs associated with sufficient hardware for large studies. Next, findings from studies that isolate low-level features of a robot system may not be generalizable, because these manipulations may not be representative of the abstract design element. For example, studies of gaze behavior often interchange eye gaze, head orientation, and their combined behavior based on the level of fidelity in which gaze mechanisms are designed in a robot system or the stylized representation of gaze chosen for the design. However, whether or not findings obtained from a study that manipulates head orientation to understand the effects of gaze on user attention would generalize to other forms of gaze is unknown. Second, system-level studies of embodiment often involve comparisons across ontologically different systems that may afford different design variables, and comparisons at the design-variable level may not be feasible. For example, a study on the effects of touch cannot compare a physical and virtual embodiment, as the latter does not afford physical touch, and techniques to simulate touch may not effectively represent natural human experience.

Another limitation that is common in studies reviewed here is the relatively small sample sizes employed and the underpowered findings that may be obtained. Several reasons underlie 
this limitation. First, the nascent state of robotics in general and socially interactive robotics in particular limits the ability to utilize some of the well established practices of empirical research, such as power analysis due to a lack of prior research that would aid in estimating expected effect sizes. Second, conducting studies with complex, potentially unreliable, and often prototype systems imposes a high cost on conducting large numbers of trials. Third, the complex, interdependent, and often fluid (due to technological advancements) design spaces of these systems require a large number of system-level and variable-level studies and motivate the use of small, rapid, and iterative experimentation. Finally, the domain-driven nature of the development of robot systems often requires sampling from special populations, such as individuals with social deficits due to developmental disorders or trauma, that may show high variability in behavior or characteristics, may have clinical demands such as the presence of a therapist, or may be difficult to recruit. While single-subject studies and qualitative studies are appropriate to study robot systems with these populations, these research paradigms are not widely adopted by the research community.

Studies that seek an understanding of human attitudes toward, perceptions of, and experience with robot systems still lack appropriate and reliable survey instruments for measurement. While some scales have been developed by the community, the validity and reliability of these instruments have not been established. Adaptations of instructions from other fields, including social psychology, interpersonal communication, and human factors, do not always result in appropriate or valid measures. For example, a two-item scale of "mutual liking" developed for studying dyads that asks the members of the dyad how much they liked their partner and think that their partner likes them and that reliably provides highly correlated results may not be appropriate in the context of studying human-robot interaction due to the ontologically asymmetrical nature of the interaction.

Finally, the use of qualitative research paradigms, methods, and measures is still rare in research in socially interactive robotics despite their potential for deeper understanding of human interaction and experience with robot systems, particularly in naturalistic settings and when quantitative methods are inappropriate. The human-computer interaction (HCI) research community has adopted and uses a wide range of qualitative paradigms and methods with success and can serve as a model for research in socially interactive robotics. Studies that are naturalistic in their entirety or those that utilize qualitative data for triangulation are essential for exploring the effects of robot systems that are integrated in human environments.

\section{Implications for Designing Embodiment}

As mentioned in previous sections, socially interactive robotics is a highly interdisciplinary field that draws from a broad variety of fields, including more mature fields such as product 
design, human-computer interaction (HCI), and mechatronics. Designers in these fields use various techniques to implement functional designs, including heuristic evaluation, user testing, critical-path analysis, and iterative design. In socially interactive robotics, designers not only have to consider the expected challenges, such as cognition, processing, perception, manipulation, locomotion, and HRI, but also the new challenges introduced by social interaction (Breazeal, 2004; Dautenhahn, 1997; Fong et al., 2003). Fong et al. (2003) introduced four design issues unique to socially interactive robots.

- Human-Oriented Perception: Socially interactive robots must have the ability to actively perceive and accurately interpret human activity and behavior (Fong et al., 2003).

- Natural HRI: Socially interactive robots must display believable behaviors, establish appropriate expectations, manage social interactions with their users, and follow human social norms (Fong et al., 2003).

- Readable Social Cues: Socially interactive robots must be able to (1) provide feedback about their internal states and (2) allow humans to interact with them in a facile, transparent manner, for example, using facial expressions, body and pointing gestures, and vocalization.

- Real-Time Performance: Socially interactive robots must operate at human interaction rates. Such robots need to simultaneously exhibit competent behavior, convey attention and intention, and handle social interaction.

The rapidly growing body of work in socially interactive robotics is providing data and insights to guide the selection and development of robot platforms for new studies and deployments. Regardless of whether researchers decide to use an off-the-shelf platform, modify an existing robot, or build an entirely new robot platform, the aggregated results from past studies can inform and facilitate the robot-selection and design processes.

The results of the reviewed studies not only highlight the benefits of physical embodiment in many different task contexts, but the aggregated evaluations also revealed patterns that can guide the design of socially interactive robots (Table A.2). By first formalizing both the application and design space of these robots and then grouping experimental design and results, we aim to provide a structured process for designing socially interactive robots informed by results from prior studies.

Figure 6.1 shows the general overview of our recommended approach for designing an application-specific robot: given a task or application, make an educated decision on what role the robot should play in that task, then select the design inspiration and set of behaviors for that robot, and then finally implement the design metaphor in the most appropriate level of abstraction. We will now discuss how we can use the results of our 


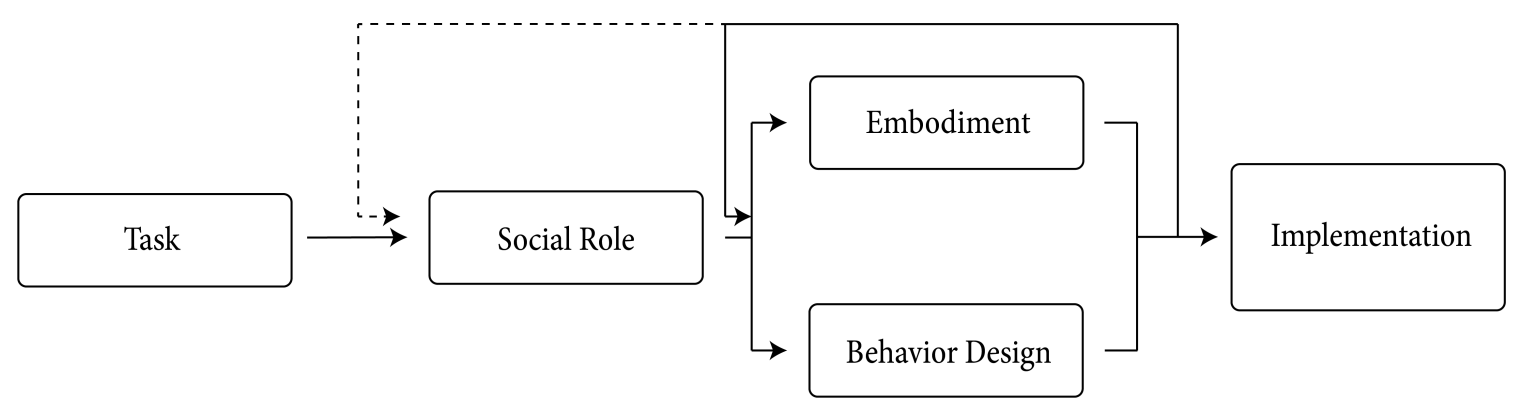

Figure 6.1: A characterization of the process for designing or selecting socially interactive robots for different tasks.

survey as well as existing literature in tangential fields to advise design decisions throughout this whole pipeline.

\subsection{Selecting Social Roles}

We have discussed socially interactive robots and their applications in the context of (1) tasks, (2) social roles, and (3) embodiment. Following Figure 6.1, we first consider the robot's task and, based on that task, select a social role for the robot. Properly selecting the role is critical because it is closely tied to a robot's ability and approach to achieving its goals; for instance, a superior robot may be a more effective teacher or coach based on heightened perceived authority (Bainbridge et al., 2011); a peer-like robot may be more engaging for a competitive task (Jost et al., 2012b); and a subordinate robot may help in improving self-efficacy (Fischer et al., 2012) or encouraging empathy (Elaine S. Short and Matarić, 2017). While selecting the and roles is still a process that requires intuition, data from the ever-growing body of past work can be used to inform the process.

Based on the reviewed studies, we can advise decisions on the social role that the robot may most effectively play in the context of that task. As the social role of a robot is a design parameter assigned by the designer, the distribution of social roles used across different task categories are a representation of the general intuition of researchers, resulting in an uneven distribution of experiments across task categories as seen in Figure 6.1. The performance of the robots playing these roles can then be used to predict potential performance of other robots for similar tasks.

\subsection{Designing Robot Embodiment}

After selecting a social role to be implemented for a socially interactive robot, the designer must develop two components: the robot's embodiment and its behaviors. Both are critical 

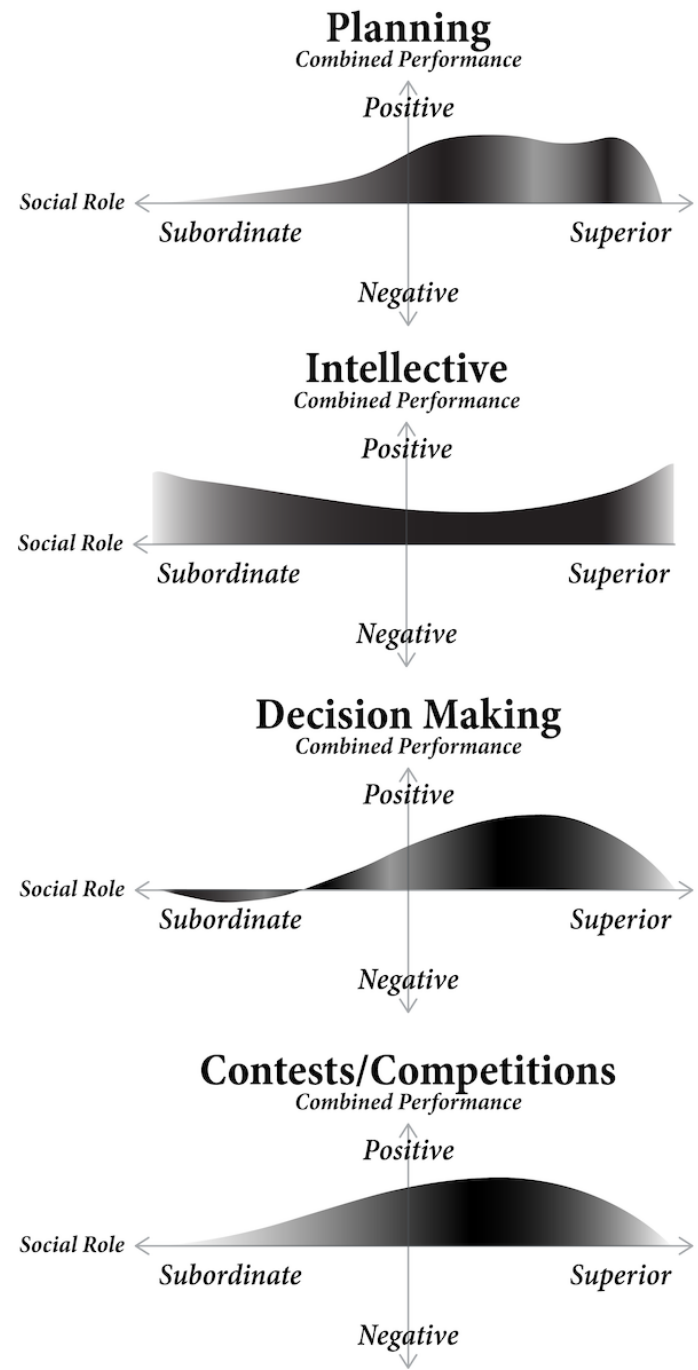
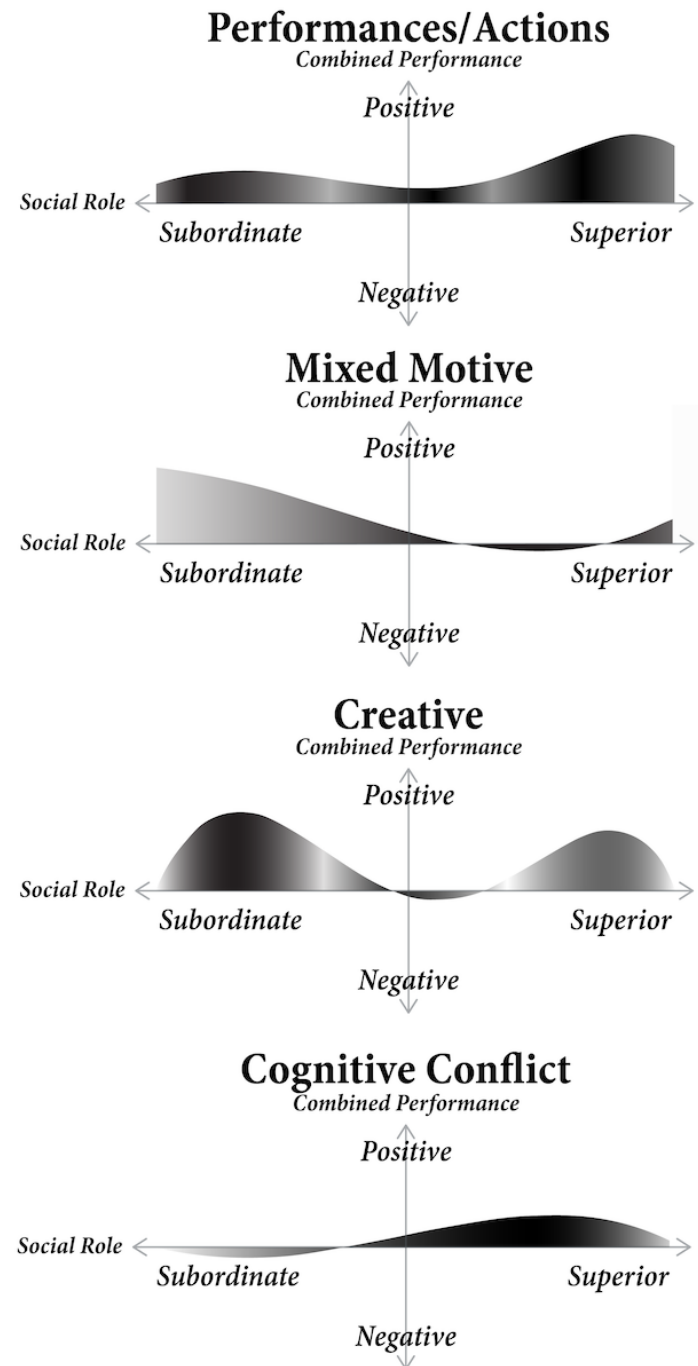

Figure 6.2: Visualization of artificial agents used for different task categories in the reviewed studies. Performance is combined performance as defined in Section 4.3.

for successfully implementing a robot's desired social role; in the context of this paper, we focus on the embodiment.

Using our previously-discussed representation of robot embodiment consisting of design metaphor and level of abstraction, we can use existing studies to advise the design or selection of robots to be used in future work (Deng et al., 2018). In our analysis of the 65 studies, we classified robot systems with the design metaphor and a level of abstraction (a numerical value of 1 to 10, smaller mapping to more abstract), seen in Table A.1. Figure 
6.3 shows the implemented social roles of robots in the reviewed studies, for each design metaphor and by levels of abstraction.

Because of the differing mental models of the various design metaphors, the same social role can be implemented with different design metaphors by changing the levels of abstraction for each design metaphor. For example, if a superior social role is desired, we can reference Figure 6.3 to find that implementing a metaphoric human form, a slightly literal bird form, or a literal car form may all effectively achieve the specific goal. Because of this flexibility, if a robot designer is constrained by either the design metaphor or the level of abstraction, they can reference existing data to advise the selection of the unconstrained design dimension and more effectively explore and iterate through the space.

\subsubsection{End-to-End Design of Socially Interactive Robots}

Using our characterization of the design space for socially interactive robots and the metaresults of the reviewed studies, we believe that the approach we present above can be effective in advising the design of new robots or selection of existing platforms for desired applications. The reviewed studies demonstrated that, for a given task, there are likely multiple social roles that robots can take. Similarly, the reviewed experiments demonstrated that the same social roles can be effectively implemented with different combinations of design metaphors and levels of abstraction.

Because "optimality" of socially interactive robot design is not a precise, quantitative process, the discussed approach can be applied to design or select not only robot embodiments for singular tasks but also for sets of tasks: first finding interaction strategies that have been shown to be effective for an individual tasks and then mapping those strategies to social roles and selecting the social role that is most effective for most (if not all) of the desired tasks (Kalegina et al., 2018; Deng et al., 2018). That social role can then be implemented and evaluated with a variety of design metaphors and levels of abstraction within the set of selected tasks.

\subsubsection{Robot Embodiment Design in Practice}

Our formalization of the design and task spaces for socially interactive robots allows us to discuss and explore how robots are designed and used. We proposed a design process that defines the order in which design features should be decided and iterated on. Finally, using data from past research studies, we proposed two ways of visualizing and leveraging experimental data to drive future design decisions-specifically considering (1) the relationship between social roles of artificial agents used for different types of tasks and (2) the mapping of differing levels of abstraction to social roles within each design metaphor. To demonstrate how these steps come together in the design of new robots or the selection of an existing 
Bird

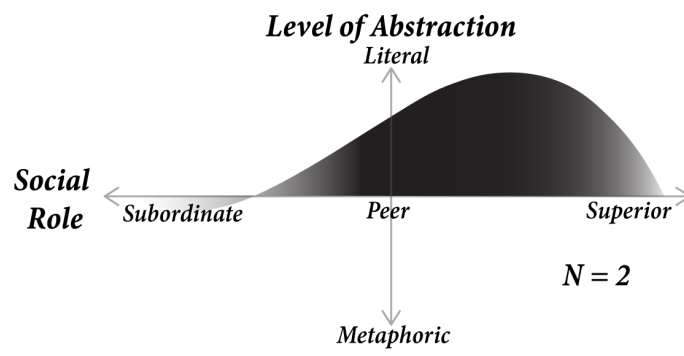

Dog

Level of Abstraction

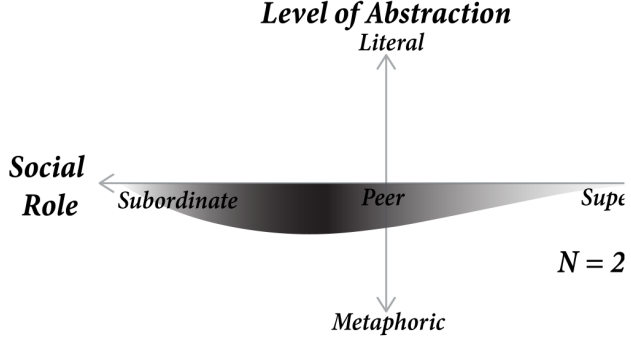

Car

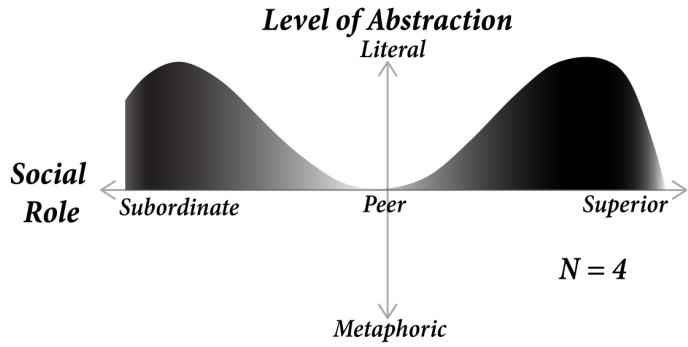

Kiosk

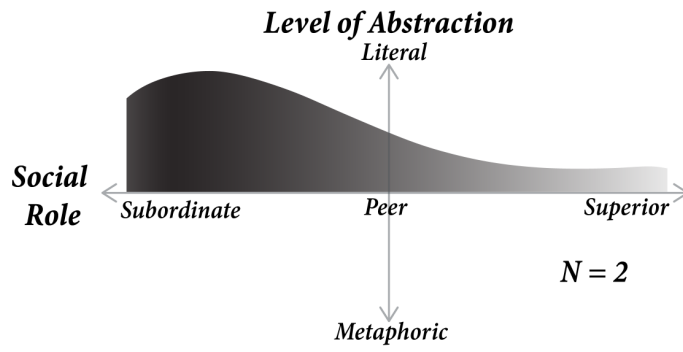

Bear

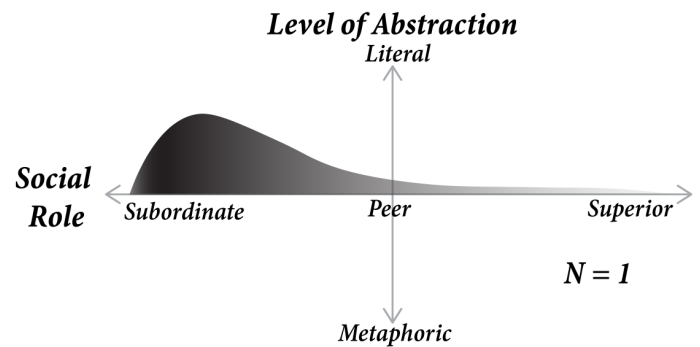

Human

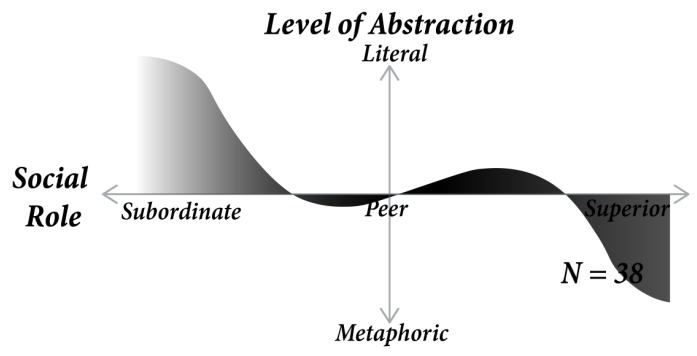

Cat

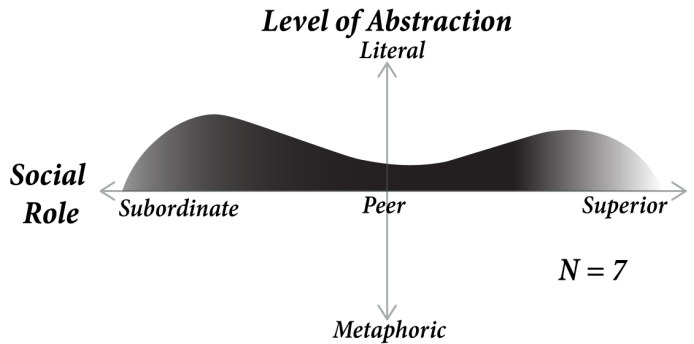

Rabbit

Level of Abstraction

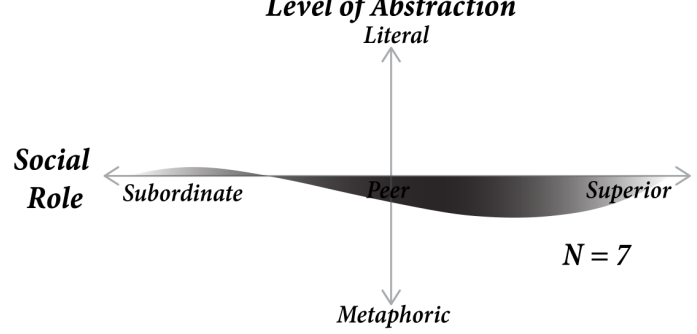

Figure 6.3: Visualization of the level of abstraction and social role by design metaphor for the systems in the reviewed studies.

robot for a new task, we provide an example. While in the examples we do not iterate on any of the design decisions, as that requires evaluation and testing, in Figure 6.1 we show where such iteration would take place on different design dimensions. 


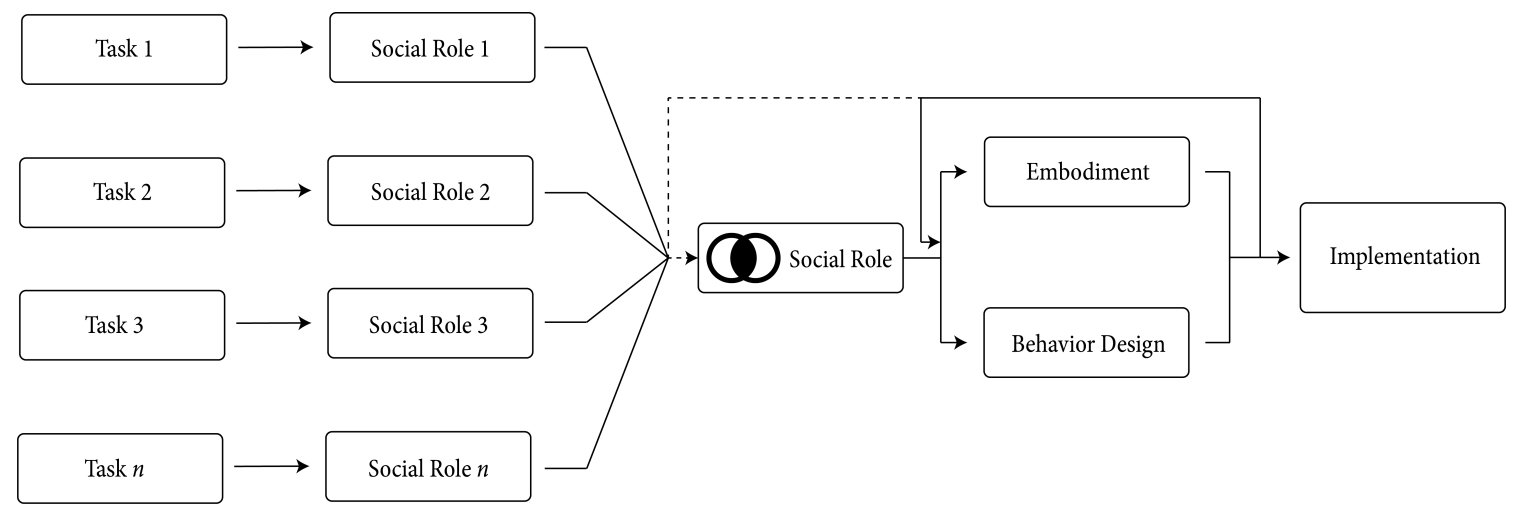

Figure 6.4: A characterization of the process for designing or selecting socially interactive robots for multiple tasks.

\section{Decision Making}

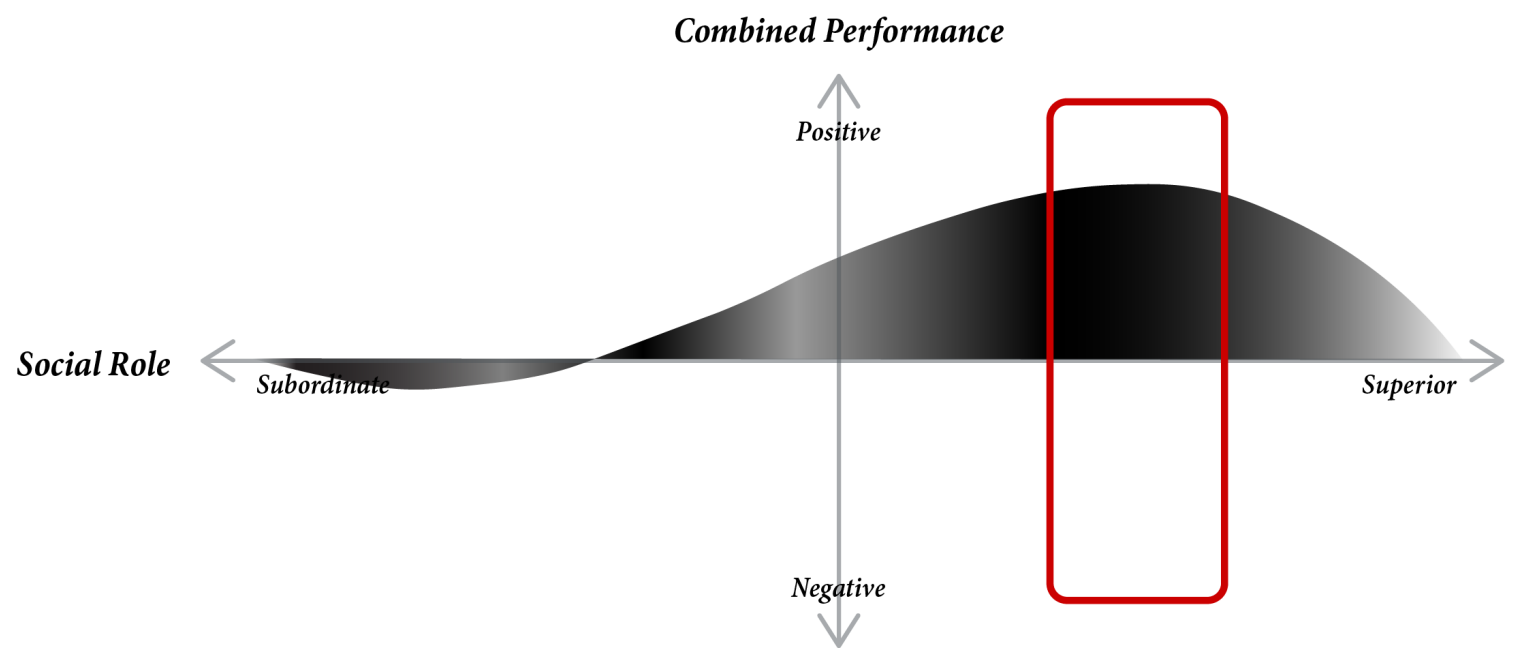

Figure 6.5: A visualization of the results from experiments with decision-making tasks plotted over the social role taken by the artificial agents and the combined performance within those experiments.

\section{Example: Grocer Store Robot}

Consider the example problem of needing to design a robot that helps people decide what to purchase in a grocery store. The robot needs to help people weigh the benefits of different food options against costs of those items. There is no objectively "best" combination of foods because features of foods can be more or less important to different people and some people may be more sensitive to price than others. Given that problem statement, this problem falls under the decision-making task category. 


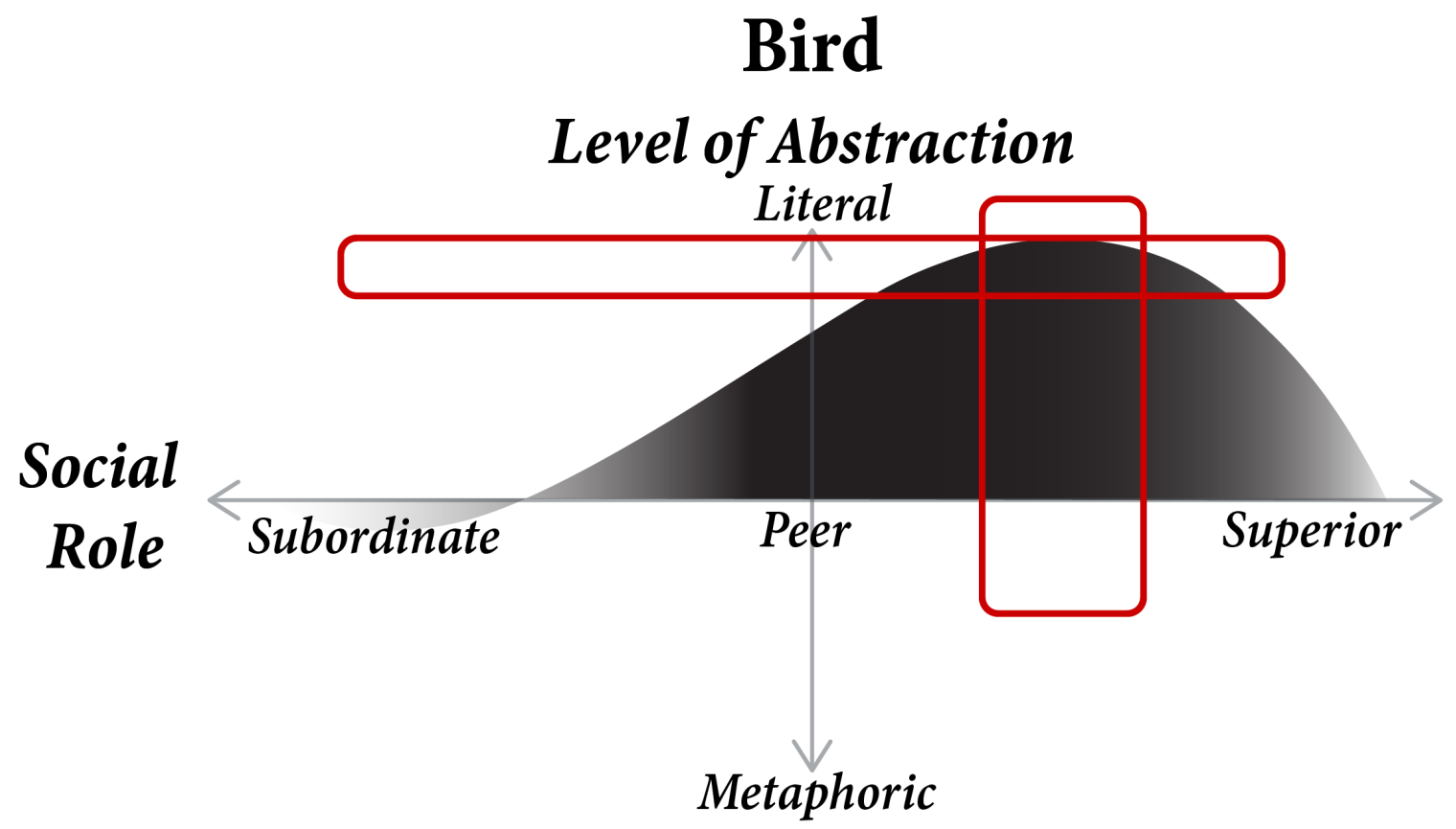

Figure 6.6: A visualization of the results from experiments with decision-making tasks plotted over the social role taken by the artificial agents and the combined performance within those experiments.

Taking into account the results from the surveyed experiments, we aim to make an educated guess as to the social role(s) to explore for the grocery store robot. Figure 6.5 shows the distribution of the decision-making task experiments plotted over performance and social role (with density of experiments shown by the overlaid gradient). The experimental results show, with relatively high confidence, that an agent with a social role somewhere between peer and superior seems to be the best choice. Given that, we then proceed to implement that social role into a robot embodiment, per Figure 6.1.

Because a social role can be implemented with different combinations of design metaphor and level of abstraction, we consider past effective design choices. The gradient in Figure 6.3 shows that birds, humans, cats and cars have been most frequently used for the peer-superior role. For this thought experiment, we arbitrarily select the bird design metaphor to start with although in real applications it is best to iterate on design metaphors and select one based on other external constraints such as physical requirements for the robot.

The bird metaphor, like all other design metaphors, can be used to implement any social role with varying levels of efficacy. Based on reviewed robots and their usage, we visualize how the bird metaphor has been previously used (Figure 6.6) and find that previous experiments have used a literal instantiation of the bird design metaphor to implement a peer-superior role. Thus we select an existing robot fitting that metaphor and abstraction combination 
(from a database such as Table 6.1) or design a new robot to fit that combination. The behaviors of the robot should then map to the appropriate levels of abstraction by changing how much it differs from the behaviors of the organic version of the metaphor.

Because the current dataset of embodiment-related experiments in the field of socially interactive robots is limited, we use all the data from studies covered in the review. The design goal can be more specific than a task-there may also be target user populations (e.g., children or elderly adults) or target demographic populations (e.g., different countries or cultures). Filtering results by those additional qualifiers (Table A.4) allows for further tailoring the data to specific problem and context.

The characterization of the design space for socially interactive robots introduced in this work aims to facilitate a concrete discussion of past work, inform the selection and development of new robots for different applications, advise future experimental design, inspire novel applications, and help to improve the iterative design process of future robots, as the field of robotics continues to expand into new avenues of use.

\section{A Reviewed Studies}


Table 4.1: Measures and instruments used to capture participant perceptions of robots in the reviewed studies.

\begin{tabular}{|c|c|c|}
\hline Instrument & Measure & Reference \\
\hline $\begin{array}{l}\text { User Acceptance of Information } \\
\text { Technology (UTAUT) }\end{array}$ & Acceptance & (Venkatesh et al., 2003) \\
\hline $\begin{array}{l}\text { Positive and Negative } \\
\text { Affect Schedule (PANAS) }\end{array}$ & Affective State & (Watson et al., 1988) \\
\hline Godspeed Questionnaire & $\begin{array}{l}\text { Anthropomorphism, } \\
\text { Animacy, Likeability, } \\
\text { Perceived Intelligence, } \\
\text { Perceived Safety }\end{array}$ & (Bartneck et al., 2009) \\
\hline $\begin{array}{l}\text { Animated Character and } \\
\text { Interface Evaluation }\end{array}$ & $\begin{array}{l}\text { Anxiety, } \\
\text { Task Performance, } \\
\text { Liking }\end{array}$ & (Rickenberg and Reeves, 2000) \\
\hline $\begin{array}{l}\text { Negative Attitudes towards } \\
\text { Robots Scale (NARS) }\end{array}$ & $\begin{array}{l}\text { Attitude, } \\
\text { Perceived Presence }\end{array}$ & (Nomura et al., 2006) \\
\hline $\begin{array}{l}\text { Questionnaire for } \\
\text { Placement Committees }\end{array}$ & Cognitive Development & (Fridin and Belokopytov, 2014) \\
\hline $\begin{array}{l}\text { NASA Task Load } \\
\text { Index Questionnaire }\end{array}$ & Cognitive Load & (Hart, 2006) \\
\hline Cognitive Load Questionnaire & Cognitive Load & (Sweller, 1988) \\
\hline $\begin{array}{l}\text { Self Assessment Manikin } \\
\text { and Semantic Differential }\end{array}$ & Emotional State & (Bradley and Lang, 1994) \\
\hline $\begin{array}{l}\text { Hoonhout Enjoyability } \\
\text { Scale }\end{array}$ & Enjoyability & (Hoonhout, 2002) \\
\hline $\begin{array}{l}\text { Adjective-Based } \\
\text { Rating }\end{array}$ & Enjoyability & $\mathrm{N} / \mathrm{A}$ \\
\hline Likert-Scale Evaluations & General & (Likert, 1932) \\
\hline UCLA Loneliness Scale & Loneliness & (Russell, 1996) \\
\hline $\begin{array}{l}\text { Standardized Mini-Mental } \\
\text { State Examination }\end{array}$ & $\begin{array}{l}\text { Mental State, } \\
\text { Development }\end{array}$ & (Crum et al., 1993) \\
\hline Kidd and Breazeal Questionnaire & Perceived Presence & (Kidd and Breazeal, 2004) \\
\hline $\begin{array}{l}\text { Interactive Experiences } \\
\text { Questionnaire }\end{array}$ & Perceived Presence & (Lombard et al., 2000) \\
\hline Eysenck Personality Questionnaire & Personality & (Francis et al., 1992) \\
\hline Big Five Questionnaire & Personality & (Caprara et al., 1993) \\
\hline "I'm Sorry Dave" Questionnaire & Sociability & (Takayama and Pantofaru, 2009) \\
\hline $\begin{array}{l}\text { Children's Social Behavior } \\
\text { Questionnaire (CSBQ) }\end{array}$ & $\begin{array}{l}\text { Social Behaviors, } \\
\text { Empathetic Abilities }\end{array}$ & (Hartman et al., 2006) \\
\hline $\begin{array}{l}\text { Networked Minds Questionnaire } \\
\text { of Social Presence }\end{array}$ & Social Presence & (Biocca et al., 2003) \\
\hline
\end{tabular}


Table 4.2: Measures of individual behavior used in the reviewed studies and methods for their capture and computation.

\begin{tabular}{|c|c|c|}
\hline Measure & Analysis Tool & Example Experiment(s) \\
\hline $\begin{array}{l}\text { Attachment Level } \\
\text { of Speech }\end{array}$ & Automated System & (Bremner and Leonards, 2015) \\
\hline Response Time & $\begin{array}{l}\text { Automated Systems, } \\
\text { Human Annotation }\end{array}$ & (Bainbridge et al., 2011) \\
\hline Directed Gaze & Human Annotation & $\begin{array}{l}\text { (Donahue and Scheutz, 2015; } \\
\text { Ju and Sirkin, 2010; Kidd and } \\
\text { Breazeal, 2004; Komatsu, 2010; } \\
\text { Williams and Breazeal, 2013) }\end{array}$ \\
\hline Facial Affect & $\begin{array}{l}\text { FACS Coding (Ekman and } \\
\text { Friesen, 1975) Tools }\end{array}$ & $\begin{array}{l}\text { (Costa et al., 2016; Shahid et al., } \\
\text { 2014; Williams and Breazeal, 2013; } \\
\text { Elaine S. Short and Matarić, 2017) }\end{array}$ \\
\hline Face Tracking & $\begin{array}{l}\text { FaceAPI, } \\
\text { Microsoft Kinect, } \\
\text { OpenFace (Amos et al., 2016) }\end{array}$ & (Costa et al., 2016) \\
\hline Micro Behaviors & Human Annotation & (Donahue and Scheutz, 2015) \\
\hline $\begin{array}{l}\text { Linguistic Verbosity/ } \\
\text { Breadth of Disclosure }\end{array}$ & Human Annotation & (Fischer et al., 2012) \\
\hline $\begin{array}{l}\text { Conversational } \\
\text { Expressiveness }\end{array}$ & Human Annotation & (Heerink et al., 2010) \\
\hline Body Pose/ & Automated Systems, & $\begin{array}{l}\text { (Jost et al., 2014; Brooks et al., } \\
2012)\end{array}$ \\
\hline Joint Positions & $\begin{array}{l}\text { Microsoft Kinect, } \\
\text { RGBD Cameras, } \\
\text { Vicon Motion Capture, } \\
\text { Human Annotation }\end{array}$ & \\
\hline
\end{tabular}


Table 4.3: Interaction measures from observed measures used in reviewed studies.

\begin{tabular}{lll} 
Measure & Analysis Tool & Example Experiment(s) \\
$\begin{array}{l}\text { Directed Gaze } \\
\text { Movement }\end{array}$ & $\begin{array}{l}\text { Human Annotation, } \\
\text { Automated Systems }\end{array}$ & (Lohan et al., 2010) \\
\hline Hutual Gaze & Human Annotation, & 2006) \\
\hline Embodied & Automated Systems & (Costa et al., 2016; Williams and \\
& Human Annotation & Breazeal, 2013; Elaine S. Short \\
Nonverbal Gestures & & and Matarić, 2017) \\
\hline Eye Contact & Human Annotation & (Fridin and Belokopytov, 2014) \\
\hline Interactivity & Human Annotation & (Kidd and Breazeal, 2004) \\
\hline Perceived Preference & Human Annotation & (Kose-Bagci et al., 2009) \\
\hline Engagement & Human Annotation & (Powers et al., 2007) \\
\hline Self-Disclosure & Human Annotation & (Powers et al., 2007) \\
\hline $\begin{array}{l}\text { Attention Directing } \\
\text { Behaviors }\end{array}$ & Human Annotation & (Looije et al., 2012) \\
\hline Advise-Seeking & Human Annotation & (Pan and Steed, 2016) \\
Behaviors & Human Annotation & (Robins et al., 2006) \\
\hline Social Touch & &
\end{tabular}


Table 6.1: Robots used in reviewed studies labeled with the design metaphor and level of abstraction that they were assigned.

\begin{tabular}{|c|c|c|c|}
\hline Robot & $\begin{array}{l}\text { Design } \\
\text { Metaphor }\end{array}$ & $\begin{array}{l}\text { Level of } \\
\text { Abstraction }\end{array}$ & Studies Used \\
\hline $\begin{array}{ll}\text { Keio U Robot- } \\
\text { phone }\end{array}$ & Bear & 4 & (Ligthart and Truong, 2015) \\
\hline Pioneer 2DX & Car & 8 & $\begin{array}{l}\text { (Donahue and Scheutz, 2015; Wainer et al., } \\
2006,2007 \text { ) }\end{array}$ \\
\hline Pioneer P3AT & Car & 8 & (Segura et al., 2012) \\
\hline iCat & Cat & 6 & $\begin{array}{l}\text { (Bartneck et al., 2004; Heerink et al., 2010; } \\
\text { Leite et al., 2008; Looije et al., 2010) } \\
\text { (Shahid et al., 2014; Pereira et al., 2008; } \\
\text { Leyzberg et al., 2012) }\end{array}$ \\
\hline Keepon & Chick & 2 & (Leyzberg et al., 2012) \\
\hline Sony Aibo & Dog & 4 & (Jung and Lee, 2004; Lee et al., 2006) \\
\hline Aesop Robot & Human & 8 & (Costa et al., 2016) \\
\hline Aldebaran NAO & Human & 6 & $\begin{array}{l}\text { (Fridin and Belokopytov, 2014; Bremner and } \\
\text { Leonards, 2015; Jost et al., 2014; Kennedy } \\
\text { et al., 2015) } \\
\text { (Krogsager et al., 2014; Ligthart and Truong, } \\
\text { 2015; Looije et al., 2012) }\end{array}$ \\
\hline Bandit & Human & 5 & (Fasola and Mataric, 2013; Tapus et al., 2009) \\
\hline Darwin-OP & Human & 6 & (Brooks et al., 2012) \\
\hline eMuu & Human & 1 & (Bartneck, 2003) \\
\hline Honda ASIMO & Human & 4 & (Takeuchi et al., 2006) \\
\hline $\mathrm{iCub}$ & Human & 7 & (Lohan et al., 2010; Fischer et al., 2012) \\
\hline KASPAR & Human & 4 & (Kose-Bagci et al., 2009) \\
\hline Kondo Kagaku & Human & 3 & (Hasegawa et al., 2010) \\
\hline MIT Robot Head & Human & 5 & (Kidd and Breazeal, 2004) \\
\hline MIT AIDA & Human & 2 & (Williams and Breazeal, 2013) \\
\hline Nico Robot & Human & 3 & (Bainbridge et al., 2011) \\
\hline Nursebot & Human & 4 & (Kiesler et al., 2008; Powers et al., 2007) \\
\hline PaPeRo & Human & 2 & (Komatsu, 2010) \\
\hline Robata & Human & 6 & (Robins et al., 2006) \\
\hline Robothespian & Human & 7 & (Pan and Steed, 2016) \\
\hline Robotis Bioloid & Human & 5 & (Jost et al., 2012b) \\
\hline Robovie-X & Human & 7 & (Nomura and Sasa, 2009) \\
\hline Samsung April & Human & 6 & (Jung and Lee, 2004; Lee et al., 2006) \\
\hline $\begin{array}{l}\text { Stanford Kiosk } \\
\text { Robot }\end{array}$ & Kiosk & 10 & (Ju and Sirkin, 2010) \\
\hline Robulab & Penguin & 7 & (Wrobel et al., 2013) \\
\hline Nabaztag & Rabbit & 3 & (Hoffmann and Krämer, 2013; Zlotowski, 2010) \\
\hline NTT Lab Robot & Rabbit & 5 & $\begin{array}{l}\text { (Shinozawa et al., 2003; Shinozawa and Yam- } \\
\text { ato, 2007) }\end{array}$ \\
\hline
\end{tabular}


Table A.1: Reviewed studies labeled with the task category and social role of artificial agents used. Papers with multiple experiments are labeled with Exp. 1, 2, etc. and social role labeled with numeric scale of 1 (subordinate) to 9 (superior).

\begin{tabular}{|c|c|c|c|}
\hline Author (Year) & Task Category & Social Role & Reference \\
\hline Bainbridge et al. (2011), Exp. 1 & Performances/Actions & Superior/Peer (9) & (Bainbridge et al., 2011) \\
\hline Bainbridge et al. (2011), Exp. 2 & Performances/Actions & Superior/Peer (9) & (Bainbridge et al., 2011) \\
\hline Bainbridge et al. (2011), Exp. 3 & Performances/Actions & Superior/Peer (9) & (Bainbridge et al., 2011) \\
\hline Bartneck (2003) & Contests/Competition & Peer $(5)$ & (Bartneck, 2003) \\
\hline Bartneck et al. (2004) & Intellective & Subordinate/Peer (3) & (Bartneck et al., 2004) \\
\hline Bremner and Leonards (2015) & Decision-Making & Subordinate/Peer (3) & (Bremner and Leonards, 2015) \\
\hline Brooks et al. (2012) & Performances/Actions & Superior/Peer (8) & (Brooks et al., 2012) \\
\hline Costa (2016), Exp. 1 & Creative & Superior/Peer (8) & (Costa et al., 2016) \\
\hline Costa (2016), Exp. 2 & Creative & Superior/Peer (8) & (Costa et al., 2016) \\
\hline Donahue and Scheutz (2015) & Performances/Actions & Subordinate (1) & (Donahue and Scheutz, 2015) \\
\hline Fasola and Mataric (2013) & Performances/Actions & Superior/Peer (8) & (Fasola and Mataric, 2013) \\
\hline Fischer et al. (2012), Exp. 1 & Intellective & Subordinate/Peer (2) & (Fischer et al., 2012) \\
\hline Fischer et al. (2012), Exp. 2 & Intellective & Subordinate/Peer (2) & (Fischer et al., 2012) \\
\hline Fischer et al. (2012), Exp. 3 & Intellective & Subordinate/Peer (2) & (Fischer et al., 2012) \\
\hline Fridin and Belokopytov (2014) & Intellective & Superior/Peer (7) & (Fridin and Belokopytov, 2014) \\
\hline Hasegawa et al. (2010) & Intellective & Superior/Peer (8) & (Hasegawa et al., 2010) \\
\hline Heerink et al. (2010) & Performances/Actions & Peer/Subordinate (2) & (Heerink et al., 2010) \\
\hline Hoffmann and Krämer (2013), Exp. 1 & Creative & Peer (5) & (Hoffmann and Krämer, 2013) \\
\hline Hoffmann and Krämer (2013), Exp. 2 & Intellective & Peer/Superior (6) & (Hoffmann and Krämer, 2013) \\
\hline Jost et al. (2014) & Intellective & Peer/Superior (6) & (Jost et al., 2014) \\
\hline Jost et al. (2012), Exp. 1 & Contests/Competition & Peer $(5)$ & (Jost et al., 2012a) \\
\hline Jost et al. (2012), Exp. 2 & Contests/Competition & Peer (5) & (Jost et al., 2012a) \\
\hline Ju and Sirkin (2010), Exp. 1 & Performances/Actions & Subordinate (1) & (Ju and Sirkin, 2010) \\
\hline Ju and Sirkin (2010), Exp. 2 & Performances/Actions & Subordinate (1) & (Ju and Sirkin, 2010) \\
\hline Jung and Lee (2004), Exp. 1 & Creative & Peer/Subordinate (2) & (Jung and Lee, 2004) \\
\hline Jung and Lee (2004), Exp. 2 & Creative & Peer/Subordinate (2) & (Jung and Lee, 2004) \\
\hline
\end{tabular}


Table A.1: Continued

\begin{tabular}{|c|c|c|c|}
\hline Author (Year) & Task Category & Social Role & Reference \\
\hline Kidd and Breazeal (2004), Exp. 2 & Creative & Superior/Peer (7) & (Kidd and Breazeal, 2004) \\
\hline Komatsu et al. (2010), Exp. 1 & Intellective & Peer/Subordinate (3) & (Komatsu, 2010) \\
\hline Komatsu et al. (2010), Exp. 2 & Intellective & Peer/Subordinate (3) & (Komatsu, 2010) \\
\hline Kose et al. (2009) & Performances/Actions & Peer $(5)$ & (Kose-Bagci et al., 2009) \\
\hline Lee et al. (2006), Exp. 2 & Creative & Peer (5) & (Lee et al., 2006) \\
\hline Lee et al. (2015) & Decision Making & Peer/Subordinate (4) & (Lee et al., 2015) \\
\hline Leite et al. (2008) & Cognitive Conflict & Peer $(5)$ & (Leite et al., 2008) \\
\hline Levy-Tzedek et al. (2017) & Performances/Actions & Superior/Peer (7) & (Levy-Tzedek et al., 2017) \\
\hline Leyzberg et al. (2012) & Contests/Competition & Peer/Superior (7) & (Leyzberg et al., 2012) \\
\hline Li and Chignell (2011) & Decision Making & Subordinate (1) & (Li and Chignell, 2011) \\
\hline Nomura (2009) & Performances/Actions & Superior/Peer (8) & (Nomura and Sasa, 2009) \\
\hline Pan and Steed (2016) & Cognitive Conflict & Superior/Peer (8) & (Pan and Steed, 2016) \\
\hline Pereira et al. (2008) & Cognitive Conflict & Peer $(5)$ & (Pereira et al., 2008) \\
\hline Powers et al. (2007) & Mixed Motive & Peer/Superior (7) & (Powers et al., 2007) \\
\hline Robins et al. (2006) & Mixed Motive & Peer/Subordinate (4) & (Robins et al., 2006) \\
\hline Segura et al. (2012) & Performances/Actions & Superior/Peer (8) & (Segura et al., 2012) \\
\hline Shahid et al. (2014) & Cognitive Conflict & Peer $(5)$ & (Shahid et al., 2014) \\
\hline Shinozawa and Reeves (2003), Exp. 1 & Mixed Motive & Peer/Subordinate (4) & (Shinozawa et al., 2003) \\
\hline Shinozawa and Reeves (2003), Exp. 2 & Planning & Superior/Peer (6) & (Shinozawa et al., 2003) \\
\hline Shinozawa and Reeves (2003), Exp. 3 & Intellective & Peer $(5)$ & (Shinozawa et al., 2003) \\
\hline Shinozawa et al. (2007) & Decision Making & Peer/Superior (7) & (Shinozawa and Yamato, 2007) \\
\hline Short et al. (2017) & Creative & Subordinate/Peer (4) & (Elaine S. Short and Matarić, 2017) \\
\hline
\end{tabular}


Table A.2: The physical robot platforms and their virtual counterparts used in the reviewed studies.

\begin{tabular}{|c|c|c|}
\hline Author (Year) & Physical Agent & Virtual Agent \\
\hline Bainbridge et al. (2011), Exp. 1 & Nico & Nico \\
\hline Bainbridge et al. (2011), Exp. 2 & Nico & Live Video of Nico \\
\hline Bainbridge et al. (2011), Exp. 3 & Nico & Live Video of Nico \\
\hline Bartneck (2003) & eMuu & Virtual eMuu \\
\hline Bartneck et al. (2004) & iCat & Virtual iCat \\
\hline Bremner and Leonard (2015) & NAO & Live Video of Human \\
\hline Brooks et al, (2012) & Darwin-OP & Manoi Animation \\
\hline Costa (2014), Exp. 1 & Aesop Robot & Greta Animation \\
\hline Costa (2014), Exp. 2 & Aesop Robot & Greta Animation \\
\hline Donahue and Scheutz (2015) & Pioneer 2DX & Virtual Pioneer 2DX \\
\hline Fasola \& Mataric (2013) & Bandit & Virtual Bandit \\
\hline Fischer et al. (2012), Exp. 1 & iCub II & Akachan \\
\hline Fischer et al. (2012), Exp. 2 & iCub II & Akachan \\
\hline Fischer et al. (2012), Exp. 3 & iCub II & Akachan \\
\hline Fridin and Belokopytov (2014) & NAO & Virtual NAO \\
\hline Hasegawa et al. (2010) & Kondo Kagaku KHR2-HV & NUMACK \\
\hline Heerink et al. (2009) & iCat & IIE Annie \\
\hline Hoffmann \& Krämer (2013), Exp. 1 & Nabaztag & Virtual Nabaztag \\
\hline Hoffmann \& Krämer (2013), Exp. 2 & Nabaztag & Virtual Nabaztag \\
\hline Jost el al. (2014) & $\mathrm{NAO}$ & \\
\hline Jost et al. (2012), Exp. 1 & Robotis Bioloid & Telecom GRETA \\
\hline Jost et al. (2012), Exp. 2 & Robotis Bioloid & Telecom GRETA \\
\hline $\mathrm{Ju}$ and Sirkin (2010), Exp. 1 & Kiosk Robot with Arm & Kiosk Robot with Projected Arm \\
\hline Ju and Sirkin (2010), Exp. 2 & Kiosk Robot with Arm & Kiosk Arm with on-screen Arm \\
\hline Jung and Lee (2004), Exp. 1 & Sony Aibo & Virtual Sony Aibo \\
\hline Jung and Lee (2004), Exp. 2 & Samsung April & Virtual Samsung April \\
\hline Kennedy et al. (2015) & $\mathrm{NAO}$ & Virtual NAO \\
\hline Kidd \& Breazeal (2004), Exp. 1 & Robot Eyes & Virtual Eyes \\
\hline Kidd \& Breazeal (2004), Exp. 2 & MIT Robot Head & Virtual MIT Robot Head \\
\hline Kiesler et al. (2008) & Nursebot & Virtual Nursebot \\
\hline Komatsu et al. (2010), Exp. 1 & PaPeRo & RobotStudio \\
\hline Komatsu et al. (2010), Exp. 2 & PaPeRo & RobotStudio \\
\hline Kose et al. (2009) & KASPAR & Virtual KASPAR \\
\hline Krogsager et al. (2014) & Aldebaran NAO & Telepresent NAO \\
\hline Lee et al. (2006), Exp. 1 & Sony Aibo & Virtual Aibo \\
\hline Lee et al. (2006), Exp. 2 & April & Virtual April \\
\hline Lee et al. (2015) & Humanoid Robot & Virtual Humanoid Robot \\
\hline Leite et al. (2008) & iCat & Virtual iCat \\
\hline Levy-Tzedek et al. (2017) & Kinova Arm & Video of Kinova Arm \\
\hline Leyzberg et al. (2012) & Keepon & Video of Keepon \\
\hline Li and Chignell (2011) & Keio U Robotphone & Virtual Keio U Robotphone \\
\hline Ligthart and Truong (2015) & $\mathrm{NAO}$ & Virtual NAO \\
\hline Lohan et al. (2010) & $\mathrm{iCub}$ & Akachan \\
\hline Looije et al. (2012) & $\mathrm{NAO}$ & Virtual NAO \\
\hline Looije, Neerincx, \& Cnossen (2010) & iCat & Virtual iCat \\
\hline Nomura (2009) & Robovie-X & Virtual Robovie-X \\
\hline Pan and Steed (2016) & Robothespian & Virtual Human Character \\
\hline Pereira et al. (2008) & iCat robot & Virtual iCat \\
\hline Powers et al. (2007) & Nursebot & Projected Virtual Agent \\
\hline Robins et al. (2006) & Robata & Passive Robata Doll \\
\hline Segura et al. (2012) & Pioneer P3AT & Virtual P3AT Head \\
\hline
\end{tabular}


Table A.2: Continued

\begin{tabular}{lcc} 
Author (Year) & Physical Agent & Virtual Agent \\
\hline Shahid et al. (2014) & iCat & Human \\
\hline Shinozawa and Reeves (2002), Exp. 1 & NTT Lab Robot & Video of Lab Robot \\
\hline Shinozawa and Reeves (2002), Exp. 2 & NTT Lab Robot & Video of Lab Robot \\
\hline Shinozawa and Reeves (2002), Exp. 3 & NTT Lab Robot & Video of Lab Robot \\
\hline Shinozawa et al. (2007) & NTT Lab Robot & Video of Lab Robot \\
\hline Short et al. (2017) & Bandit on Pioneer & Pioneer with Bubble machine \\
\hline Takeuchi et al. (2006) & Honda ASIMO & Microsoft Peedy \\
\hline Tapus, Tapus \& Mataric (2009) & Bandit & Virtual Bandit \\
\hline Vossen et al. (2009) & iCat & Voice only \\
\hline Wainer et al. (2006) & Pioneer 2DX & Virtual Pioneer 2DX \\
\hline Wainer et al. (2007) & Pioneer 2DX & Virtual Pioneer 2DX \\
\hline Williams et al. (2013) & MIT AIDA & AIDA on-screen App \\
\hline Wrobel et al. (2013) & Robulab & Virtual Greta \\
\hline Zlotowski (2010) & Nabaztag & Virtual Nabaztag \\
\hline
\end{tabular}


Table A.3: The results of the reviewed studies broken down by task performance differences and interaction performance differences.

\begin{tabular}{|c|c|c|}
\hline Author (Year) & Task Performance & Interaction Performance \\
\hline Bainbridge et al. (2011), Exp. 1 & $\mathrm{PE}>\mathrm{VE}$ & $\mathrm{PE}>\mathrm{VE}$ \\
\hline Bainbridge et al. (2011), Exp. 2 & $\mathrm{PE}>\mathrm{VE}$ & $\mathrm{PE}>\mathrm{VE}$ \\
\hline Bainbridge et al. (2011), Exp. 3 & $\mathrm{PE}>\mathrm{VE}$ & $\mathrm{PE}>\mathrm{VE}$ \\
\hline Bartneck (2003) & $\mathrm{PE}>\mathrm{VE}$ & $\mathrm{PE}=\mathrm{VE}$ \\
\hline Bartneck et al. (2004) & $\mathrm{PE}=\mathrm{VE}$ & $\mathrm{PE}=\mathrm{VE}$ \\
\hline Bremner and Leonard (2015) & $\mathrm{PE}=\mathrm{VE}$ & N/A \\
\hline Brooks et al, (2012) & $\mathrm{PE}>\mathrm{VE}$ & $\mathrm{N} / \mathrm{A}$ \\
\hline Costa (2014), Exp. 1 & $\mathrm{PE}>\mathrm{VE}$ & $\mathrm{PE}=\mathrm{VE}$ \\
\hline Costa (2014), Exp. 2 & $\mathrm{PE}>\mathrm{VE}$ & $\mathrm{PE}=\mathrm{VE}$ \\
\hline Donahue and Scheutz (2015) & $\mathrm{PE}>\mathrm{VE}$ & N/A \\
\hline Fasola \& Mataric (2013) & $\mathrm{PE}=\mathrm{VE}$ & $\mathrm{PE}>\mathrm{VE}$ \\
\hline Fischer et al. (2012), Exp. 1 & $\mathrm{PE}>\mathrm{VE}$ & N/A \\
\hline Fischer et al. (2012), Exp. 2 & $\mathrm{PE}>\mathrm{VE}$ & $\mathrm{N} / \mathrm{A}$ \\
\hline Fischer et al. (2012), Exp. 3 & $\mathrm{PE}>\mathrm{VE}$ & N/A \\
\hline Fridin and Belokopytov (2014) & $\mathrm{PE}=\mathrm{VE}$ & $\mathrm{PE}>\mathrm{VE}$ \\
\hline Hasegawa et al. (2010) & $\mathrm{PE}=\mathrm{VE}$ & $\mathrm{PE}>\mathrm{VE}$ \\
\hline Heerink et al. (2009) & $\mathrm{N} / \mathrm{A}$ & $\mathrm{PE}>\mathrm{VE}$ \\
\hline Hoffmann \& Krämer (2013), Exp. 1 & $\mathrm{PE}=\mathrm{VE}$ & $\mathrm{PE}<\mathrm{VE}$ \\
\hline Hoffmann \& Krämer (2013), Exp. 2 & $\mathrm{PE}=\mathrm{VE}$ & $\mathrm{PE}>\mathrm{VE}$ \\
\hline Jost el al. (2014) & $\mathrm{PE}>\mathrm{VE}$ & N/A \\
\hline Jost et al. (2012), Exp. 1 & $\mathrm{PE}>\mathrm{VE}$ & $\mathrm{PE}>\mathrm{VE}$ \\
\hline Jost et al. (2012), Exp. 2 & $\mathrm{PE}>\mathrm{VE}$ & $\mathrm{PE}>\mathrm{VE}$ \\
\hline Ju and Sirkin (2010), Exp. 1 & $\mathrm{PE}>\mathrm{VE}$ & $\mathrm{PE}<\mathrm{VE}$ \\
\hline Ju and Sirkin (2010), Exp. 2 & $\mathrm{PE}>\mathrm{VE}$ & $\mathrm{PE}<\mathrm{VE}$ \\
\hline Jung and Lee (2004), Exp. 1 & N/A & $\mathrm{PE}>\mathrm{VE}$ \\
\hline Jung and Lee (2004), Exp. 2 & N/A & $\mathrm{PE}>\mathrm{VE}$ \\
\hline Kennedy et al. (2015) & $\mathrm{PE}>\mathrm{VE}$ & $\mathrm{PE}>\mathrm{VE}$ \\
\hline Kidd \& Breazeal (2004), Exp. 1 & N/A & $\mathrm{PE}>\mathrm{VE}$ \\
\hline Kidd \& Breazeal (2004), Exp. 2 & N/A & $\mathrm{PE}>\mathrm{VE}$ \\
\hline Kiesler et al. (2008) & $\mathrm{PE}>\mathrm{VE}$ & $\mathrm{PE}>\mathrm{VE}$ \\
\hline Komatsu et al. (2010), Exp. 1 & $\mathrm{PE}=\mathrm{VE}$ & $\mathrm{PE}>\mathrm{VE}$ \\
\hline Komatsu et al. (2010), Exp. 2 & $\mathrm{PE}=\mathrm{VE}$ & $\mathrm{PE}>\mathrm{VE}$ \\
\hline Kose et al. (2009) & $\mathrm{PE}>\mathrm{VE}$ & $\mathrm{PE}=\mathrm{VE}$ \\
\hline Krogsager et al. (2014) & $\mathrm{PE}<\mathrm{VE}$ & $\mathrm{PE}<\mathrm{VE}$ \\
\hline Lee et al. (2006), Exp. 1 & N/A & $\mathrm{PE}>\mathrm{VE}$ \\
\hline Lee et al. (2006), Exp. 2 & $\mathrm{~N} / \mathrm{A}$ & $\mathrm{PE}<\mathrm{VE}$ \\
\hline Lee et al. (2015) & $\mathrm{N} / \mathrm{A}$ & $\mathrm{PE}=\mathrm{VE}$ \\
\hline Leite et al. (2008) & N/A & $\mathrm{PE}>\mathrm{VE}$ \\
\hline Levy-Tzedek et al. (2017) & $\mathrm{PE}>\mathrm{VE}$ & $\mathrm{PE}>\mathrm{VE}$ \\
\hline Leyzberg et al. (2012) & $\mathrm{PE}>\mathrm{VE}$ & $\mathrm{PE}>\mathrm{VE}$ \\
\hline $\mathrm{Li}$ and Chignell (2011) & $\mathrm{PE}=\mathrm{VE}$ & $\mathrm{N} / \mathrm{A}$ \\
\hline Ligthart and Truong (2015) & N/A & $\mathrm{PE}=\mathrm{VE}$ \\
\hline Lohan et al. (2010) & $\mathrm{N} / \mathrm{A}$ & $\mathrm{PE}>\mathrm{VE}$ \\
\hline Looije et al. (2012) & $\mathrm{N} / \mathrm{A}$ & $\mathrm{PE}>\mathrm{VE}$ \\
\hline Looije, Neerincx, \& Cnossen (2010) & $\mathrm{PE}>\mathrm{VE}$ & $\mathrm{PE}<\mathrm{VE}$ \\
\hline Nomura (2009) & $\mathrm{PE}=\mathrm{VE}$ & $\mathrm{PE}=\mathrm{VE}$ \\
\hline Pan and Steed (2016) & $\mathrm{PE}>\mathrm{VE}$ & $\mathrm{PE}>\mathrm{VE}$ \\
\hline Pereira et al. (2008) & N/A & $\mathrm{PE}>\mathrm{VE}$ \\
\hline Powers et al. (2007) & $\mathrm{PE}<\mathrm{VE}$ & $\mathrm{PE}>\mathrm{VE}$ \\
\hline Robins et al. (2006) & $\mathrm{PE}>\mathrm{VE}$ & $\mathrm{PE}>\mathrm{VE}$ \\
\hline Segura et al. (2012) & $\mathrm{PE}>\mathrm{VE}$ & $\mathrm{PE}>\mathrm{VE}$ \\
\hline
\end{tabular}


Table A.3: Continued

\begin{tabular}{lcc} 
Author (Year) & Task Performance & Interaction Performance \\
\hline Shahid et al. (2014) & $\mathrm{PE}>\mathrm{VE}$ & $\mathrm{PE}>\mathrm{VE}$ \\
Shinozawa and Reeves (2002), Exp. 1 & $\mathrm{PE}>\mathrm{VE}$ & $\mathrm{PE}>\mathrm{VE}$ \\
Shinozawa and Reeves (2002), Exp. 2 & $\mathrm{PE}>\mathrm{VE}$ & $\mathrm{PE}>\mathrm{VE}$ \\
Shinozawa and Reeves (2002), Exp. 3 & $\mathrm{PE}>\mathrm{VE}$ & $\mathrm{PE}>\mathrm{VE}$ \\
Shinozawa et al. (2007) & $\mathrm{PE}>\mathrm{VE}$ & $\mathrm{PE}>\mathrm{VE}$ \\
Short et al. (2017) & $\mathrm{PE}>\mathrm{VE}$ & $\mathrm{PE}>\mathrm{VE}$ \\
Takeuchi et al. (2006) & $\mathrm{PE}>\mathrm{VE}$ & $\mathrm{PE}>\mathrm{VE}$ \\
Tapus, Tapus \& Mataric (2009) & $\mathrm{PE}>\mathrm{VE}$ & $\mathrm{PE}>\mathrm{VE}$ \\
Vossen et al. (2009) & $\mathrm{PE}>\mathrm{VE}$ & $\mathrm{PE}>\mathrm{VE}$ \\
Wainer et al. (2006) & $\mathrm{PE}>\mathrm{VE}$ & $\mathrm{PE}>\mathrm{VE}$ \\
Wainer et al. (2007) & $\mathrm{PE}>\mathrm{VE}$ & $\mathrm{PE}>\mathrm{VE}$ \\
Williams et al. (2013) & $\mathrm{PE}>\mathrm{VE}$ & $\mathrm{PE}>\mathrm{VE}$ \\
Wrobel et al. (2013) & $\mathrm{PE}>\mathrm{VE}$ & $\mathrm{PE}<\mathrm{VE}$ \\
Zlotowski (2010) & $\mathrm{PE}<\mathrm{VE}$ &
\end{tabular}


Table A.4: Demographic characteristics of the participant pools in the reviewed studies.

\begin{tabular}{|c|c|c|c|}
\hline Author (Year) & $\mathrm{n}$ & Age Group & Country \\
\hline Bainbridge et al. (2011), Exp. 1 & 59 & Adults & US \\
\hline Bainbridge et al. (2011), Exp. 2 & 59 & Adults & US \\
\hline Bainbridge et al. (2011), Exp. 3 & 59 & Adults & US \\
\hline Bartneck (2003) & 53 & Adults & Netherlands \\
\hline Bartneck et al. (2004) & 56 & Adults & Netherlands \\
\hline Bremner and Leonard (2015) & 22 & Adults & UK \\
\hline Brooks et al, (2012) & 11 & Adults & US \\
\hline Costa (2014), Exp. 1 & 20 & Adults & United Arab Emirates \\
\hline Costa (2014), Exp. 2 & 40 & Adults & United Arab Emirates \\
\hline Donahue and Scheutz (2015) & 55 & Adults & US \\
\hline Fasola \& Mataric (2013) & 33 & Elderly Adults & US \\
\hline Fischer et al. (2012), Exp. 1 & 38 & Adults & Germany \\
\hline Fischer et al. (2012), Exp. 2 & 14 & Adults & Germany \\
\hline Fischer et al. (2012), Exp. 3 & 36 & Adults & Germany \\
\hline Fridin and Belokopytov (2014) & 13 & Children & Israel \\
\hline Hasegawa et al. (2010) & 75 & Children & Japan \\
\hline Heerink et al. (2009) & 40 & Elderly Adults & Netherlands \\
\hline Hoffmann \& Krämer (2013), Exp. 1 & 83 & Adults & Germany \\
\hline Hoffmann \& Krämer (2013), Exp. 2 & 83 & Adults & Germany \\
\hline Jost el al. (2014) & 67 & Children and Adults & France \\
\hline Jost et al. (2012), Exp. 1 & 51 & Children & France \\
\hline Jost et al. (2012), Exp. 2 & 52 & Children & France \\
\hline Ju and Sirkin (2010), Exp. 1 & 179 & Adults & US \\
\hline $\mathrm{Ju}$ and Sirkin (2010), Exp. 2 & 457 & Adults & US \\
\hline Jung and Lee (2004), Exp. 1 & 36 & Adults & US \\
\hline Jung and Lee (2004), Exp. 2 & 32 & Adults & US \\
\hline Kennedy et al. (2015) & 28 & Children & $\mathrm{EU}$ \\
\hline Kidd \& Breazeal (2004), Exp. 1 & 32 & Adults & US \\
\hline Kidd \& Breazeal (2004), Exp. 2 & 82 & Adults & US \\
\hline Kiesler et al. (2008) & 113 & Adults & US \\
\hline Komatsu et al. (2010), Exp. 1 & 20 & Children & Japan \\
\hline Komatsu et al. (2010), Exp. 2 & 40 & Children & Japan \\
\hline Kose et al. (2009) & 66 & Children & UK \\
\hline Krogsager et al. (2014) & 9 & Children & Denmark \\
\hline Lee et al. (2006), Exp. 1 & 32 & Adults & US \\
\hline Lee et al. (2006), Exp. 2 & 32 & Adults & US \\
\hline Lee et al. (2015) & 24 & Adults & US \\
\hline Leite et al. (2008) & 9 & Children and Adults & Portugal \\
\hline Levy-Tzedek et al. (2017) & 22 & Adults & Israel \\
\hline Leyzberg et al. (2012) & 100 & Adults & US \\
\hline $\mathrm{Li}$ and Chignell (2011) & 16 & Adults & Japan \\
\hline Ligthart and Truong (2015) & 40 & Adults & Netherlands \\
\hline Lohan et al. (2010) & 28 & Adults & Germany \\
\hline Looije et al. (2012) & 11 & Children & Netherlands \\
\hline Looije, Neerincx, \& Cnossen (2010) & 24 & Adults & Netherlands \\
\hline Nomura (2009) & 37 & Adults & Japan \\
\hline Pan and Steed (2016) & 24 & Adults & UK \\
\hline Pereira et al. (2008) & 18 & Children & Portugal \\
\hline Powers et al. (2007) & 113 & Adults & US \\
\hline Robins et al. (2006) & 4 & Children & UK \\
\hline Segura et al. (2012) & 42 & Adults & UK \\
\hline
\end{tabular}


Table A.4: Continued

\begin{tabular}{lccc} 
Author (Year) & $\mathrm{n}$ & Age Group & Country \\
\hline Shahid et al. (2014) & 112 & Children & Netherlands and Pakistan \\
Shinozawa and Reeves (2002), Exp. 1 & 72 & Adults & Japan and US \\
Shinozawa and Reeves (2002), Exp. 2 & 72 & Adults & Japan and US \\
Shinozawa and Reeves (2002), Exp. 3 & 72 & Adults & Japan and US \\
Shinozawa et al. (2007) & 178 & Adults & Japan \\
Short et al. (2017) & 6 & Children & US \\
Takeuchi et al. (2006) & 31 & Adults & Japan \\
Tapus, Tapus \& Mataric (2009) & 3 & Elderly Adults & US \\
Vossen et al. (2009) & 76 & Adults & Netherlands \\
Wainer et al. (2006) & 11 & Adults & US \\
Wainer et al. (2007) & 21 & Adults & US \\
Williams et al. (2013) & 44 & Adults & US \\
Wrobel et al. (2013) & 19 & Adults & France \\
Zlotowski (2010) & 16 & Adults & Finland \\
\hline
\end{tabular}


Table A.5: Reviewed studies labeled with observed measures used (task performance, interaction performance, individual behavior) and whether or not self-reported measures were implemented.

\begin{tabular}{|c|c|c|}
\hline Author (Year) & Observed Measures & $\begin{array}{c}\text { Self-Reported } \\
\text { Measures }\end{array}$ \\
\hline Bainbridge et al. (2011), Exp 1 & Task Performance, Interaction Performance & Yes \\
\hline Bainbridge et al. (2011), Exp. 2 & Task Performance, Interaction Performance & Yes \\
\hline Bainbridge et al. (2011), Exp. 3 & Task Performance, Interaction Performance & Yes \\
\hline Bartneck (2003) & Task Performance & Yes \\
\hline Bartneck et al. (2004) & Task Performance & Yes \\
\hline Bremner and Leonard (2015) & Individual Behavior & No \\
\hline Brooks et al, (2012) & Task Performance, Interaction Performance & No \\
\hline Costa (2014), Exp. 1 & Individual Behavior, Interaction Performance & Yes \\
\hline Costa (2014), Exp. 2 & individual Behavior, Interaction Performance & Yes \\
\hline Donahue and Scheutz (2015) & Individual Behavior & No \\
\hline Fasola \& Mataric (2013) & Task Performance & Yes \\
\hline Fischer et al. (2012), Exp. 1 & Task Performance, Interaction Performance & No \\
\hline Fischer et al. (2012), Exp. 2 & Task Performance, Interaction Performance & No \\
\hline Fischer et al. (2012), Exp. 3 & Task Performance, Interaction Performance & No \\
\hline Fridin and Belokopytov (2014) & Task Performance, Interaction Performance & Yes \\
\hline Hasegawa et al. (2010) & Task Performance & Yes \\
\hline Heerink et al. (2009) & Individual Behavior & Yes \\
\hline Hoffmann \& Krämer (2013), Exp. 1 & Task Performance & Yes \\
\hline Hoffmann \& Krämer (2013), Exp. 2 & Task Performance & Yes \\
\hline Jost el al. (2014) & Individual Behavior, Interaction Performance & No \\
\hline Jost et al. (2012), Exp. 1 & Task Performance, Interaction Performance & Yes \\
\hline Jost et al. (2012), Exp. 2 & $\mathrm{~N} / \mathrm{A}$ & Yes \\
\hline Ju and Sirkin (2010), Exp. 1 & Task Performance & Yes \\
\hline Ju and Sirkin (2010), Exp. 2 & Task Performance & Yes \\
\hline Jung and Lee (2004), Exp. 1 & $\mathrm{~N} / \mathrm{A}$ & Yes \\
\hline Jung and Lee (2004), Exp. 2 & $\mathrm{~N} / \mathrm{A}$ & Yes \\
\hline Kennedy et al. (2015) & Task Performance & Yes \\
\hline Kidd \& Breazeal (2004), Exp. 1 & Individual Behavior, Interaction Performance & Yes \\
\hline Kidd \& Breazeal (2004), Exp. 2 & $\mathrm{~N} / \mathrm{A}$ & Yes \\
\hline Kiesler et al. (2008) & Task Performance & Yes \\
\hline Komatsu et al. (2010), Exp. 1 & Task Performance, Interaction Performance & No \\
\hline Komatsu et al. (2010), Exp. 2 & Task Performance, Interaction Performance & No \\
\hline Kose et al. (2009) & Task Performance, Interaction Performance & Yes \\
\hline Krogsager et al. (2014) & Task Performance & Yes \\
\hline Lee et al. (2006), Exp. 1 & $\mathrm{~N} / \mathrm{A}$ & Yes \\
\hline Lee et al. (2006), Exp. 2 & $\mathrm{~N} / \mathrm{A}$ & Yes \\
\hline Lee et al. (2015) & $\mathrm{N} / \mathrm{A}$ & Yes \\
\hline Leite et al. (2008) & $\mathrm{N} / \mathrm{A}$ & Yes \\
\hline Levy-Tzedek et al. (2017) & Task Performance & Yes \\
\hline Leyzberg et al. (2012) & Task Performance & Yes \\
\hline Li and Chignell (2011) & Task Performance & Yes \\
\hline Ligthart and Truong (2015) & $\mathrm{N} / \mathrm{A}$ & Yes \\
\hline Lohan et al. (2010) & Task Performance, Interaction Performance & No \\
\hline Looije et al. (2012) & Task Performance, Interaction Performance & Yes \\
\hline Looije, Neerincx, \& Cnossen (2010) & $\mathrm{N} / \mathrm{A}$ & Yes \\
\hline Nomura (2009) & Task Performance & Yes \\
\hline Pan and Steed (2016) & Task Performance, Interaction Performance & Yes \\
\hline Pereira et al. (2008) & $\mathrm{N} / \mathrm{A}$ & Yes \\
\hline Powers et al. (2007) & Task Performance, Interaction Performance & Yes \\
\hline Robins et al. (2006) & Task Performance, Interaction Performance & Yes \\
\hline Segura et al. (2012) & Task Performance, Interaction Performance & Yes \\
\hline
\end{tabular}


Table A.5: Continued

\begin{tabular}{lcc} 
Author (Year) & Observed Measures & $\begin{array}{c}\text { Self-Reported } \\
\text { Measures }\end{array}$ \\
\hline Shahid et al. (2014) & Task Performance, Interaction Performance & Yes \\
Shinozawa and Reeves (2002), Exp. 1 & Task Performance & Yes \\
Shinozawa and Reeves (2002), Exp. 2 & Task Performance & Yes \\
Shinozawa and Reeves (2002), Exp. 3 & Task Performance & Yes \\
Shinozawa et al. (2007) & Task Performance & Yes \\
Short et al. (2017) & Task Performance, Interaction Performance & Yes \\
Takeuchi et al. (2006) & N/A & Yes \\
Tapus, Tapus \& Mataric (2009) & Task Performance, Interaction Performance & Yes \\
Vossen et al. (2009) & Task Performance & Yes \\
Wainer et al. (2006) & Task Performance & Yes \\
Wainer et al. (2007) & Task Performance & Yes \\
Williams et al. (2013) & Task Performance, Interaction Performance & Yes \\
Wrobel et al. (2013) & N/A & Yes \\
Zlotowski (2010) & Task Performance & Yes \\
\hline
\end{tabular}




\section{References}

I Elaine Allen and Christopher A Seaman. Likert scales and data analyses. Quality progress, 40(7): 64, 2007.

Brandon Amos, Bartosz Ludwiczuk, and Mahadev Satyanarayanan. Openface: A general-purpose face recognition library with mobile applications. Technical report, CMU-CS-16-118, CMU School of Computer Science, 2016.

Michael L Anderson. Embodied cognition: A field guide. Artificial intelligence, 149(1):91-130, 2003.

Sean Andrist, Tomislav Pejsa, Bilge Mutlu, and Michael Gleicher. Designing effective gaze mechanisms for virtual agents. In Proceedings of the SIGCHI conference on Human factors in computing systems, pages 705-714. ACM, 2012.

Sean Andrist, Bilge Mutlu, and Michael Gleicher. Conversational gaze aversion for virtual agents. In International Workshop on Intelligent Virtual Agents, pages 249-262. Springer, 2013.

Sean Andrist, Xiang Zhi Tan, Michael Gleicher, and Bilge Mutlu. Conversational gaze aversion for humanlike robots. In Proceedings of the 2014 ACM/IEEE international conference on Human-robot interaction, pages 25-32. ACM, 2014.

Sean Andrist, Bilge Mutlu, and Adriana Tapus. Look like me: matching robot personality via gaze to increase motivation. In Proceedings of the 33rd annual ACM conference on human factors in computing systems, pages 3603-3612. ACM, 2015.

Sean Andrist, Michael Gleicher, and Bilge Mutlu. Looking coordinated: Bidirectional gaze mechanisms for collaborative interaction with virtual characters. In Proceedings of the 2017 CHI Conference on Human Factors in Computing Systems, pages 2571-2582. ACM, 2017.

Alissa N Antle. Lifelong interactions embodied child computer interaction: why embodiment matters. interactions, 16(2):27-30, 2009.

Michael Argyle. Bodily communication. 2nd. London: Methuen, page 10, 1988.

Michael Argyle and Janet Dean. Eye-contact, distance and affiliation. Sociometry, pages 289-304, 1965.

C Asfahl. Robots and manufacturing automation. John Wiley \& Sons, Inc., 1992.

Jeremy N Bailenson and Nick Yee. A longitudinal study of task performance, head movements, subjective report, simulator sickness, and transformed social interaction in collaborative virtual environments. Presence: Teleoperators and Virtual Environments, 15(6):699-716, 2006.

Jeremy N Bailenson, Jim Blascovich, Andrew C Beall, and Jack M Loomis. Equilibrium theory revisited: Mutual gaze and personal space in virtual environments. Presence, 10(6):583-598, 2001.

Wilma A Bainbridge, Justin W Hart, Elizabeth S Kim, and Brian Scassellati. The benefits of interactions with physically present robots over video-displayed agents. International Journal of Social Robotics, 3(1):41-52, 2011.

Lisa Feldman Barrett. Are emotions natural kinds? Perspectives on psychological science, 1(1): 28-58, 2006a.

Lisa Feldman Barrett. Solving the emotion paradox: Categorization and the experience of emotion. Personality and social psychology review, 10(1):20-46, 2006b. 
Lawrence W Barsalou, Paula M Niedenthal, Aron K Barbey, and Jennifer A Ruppert. Social embodiment. Psychology of learning and motivation, 43:43-92, 2003.

Christoph Bartneck. Interacting with an embodied emotional character. In Proceedings of the 2003 international conference on Designing pleasurable products and interfaces, pages 55-60. ACM, 2003.

Christoph Bartneck, Juliane Reichenbach, and van A Breemen. In your face, robot! the influence of a character's embodiment on how users perceive its emotional expressions. In Proceedings of the Design and Emotion, pages 32-51, 2004.

Christoph Bartneck, Dana Kulić, Elizabeth Croft, and Susana Zoghbi. Measurement instruments for the anthropomorphism, animacy, likeability, perceived intelligence, and perceived safety of robots. International journal of social robotics, 1(1):71-81, 2009.

Andrea Bauer, Dirk Wollherr, and Martin Buss. Human-robot collaboration: a survey. International Journal of Humanoid Robotics, 5(01):47-66, 2008.

Roger Bemelmans, Gert Jan Gelderblom, Pieter Jonker, and Luc De Witte. Socially assistive robots in elderly care: A systematic review into effects and effectiveness. Journal of the American Medical Directors Association, 13(2):114-120, 2012.

Patricia Benner. Interpretive phenomenology: Embodiment, caring, and ethics in health and illness. Sage publications, 1994.

T. Bickmore and J. Cassell. Relational agents: a model and implementation of building user trust. In Proceedings of the SIGCHI conference on Human factors in computing systems, pages 396-403. ACM, 2001.

Timothy Bickmore and Justine Cassell. Social dialongue with embodied conversational agents. In Advances in natural multimodal dialogue systems, pages 23-54. Springer, 2005.

Timothy W Bickmore and Rosalind W Picard. Establishing and maintaining long-term humancomputer relationships. ACM Transactions on Computer-Human Interaction (TOCHI), 12(2): 293-327, 2005.

Frank Biocca. The cyborg's dilemma: Progressive embodiment in virtual environments [1]. Journal of Computer-Mediated Communication, 3(2):0-0, 1997.

Frank Biocca and Kristine Nowak. Plugging your body into the telecommunication system: Mediated embodiment, media interfaces, and social virtual environments. Communication technology and society, pages 407-447, 2001.

Frank Biocca, Chad Harms, and Judee K Burgoon. Toward a more robust theory and measure of social presence: Review and suggested criteria. Presence: Teleoperators $\&$ virtual environments, 12(5):456-480, 2003.

Avrim L Blum and Pat Langley. Selection of relevant features and examples in machine learning. Artificial intelligence, 97(1):245-271, 1997.

Margaret M Bradley and Peter J Lang. Measuring emotion: the self-assessment manikin and the semantic differential. Journal of behavior therapy and experimental psychiatry, 25(1):49-59, 1994.

Cynthia Breazeal. Toward sociable robots. Robotics and autonomous systems, 42(3):167-175, 2003. 
Cynthia Breazeal and J Velasquez. Robot in society: friend or appliance. In Proceedings of the 1999 Autonomous Agents Workshop on Emotion-Based Agent Architectures, pages 18-26, 1999.

Cynthia Breazeal, Cory D Kidd, Andrea Lockerd Thomaz, Guy Hoffman, and Matt Berlin. Effects of nonverbal communication on efficiency and robustness in human-robot teamwork. In 2005 IEEE/RSJ International Conference on Intelligent Robots and Systems, pages 708-713. IEEE, 2005.

Cynthia L Breazeal. Designing sociable robots. MIT press, 2004.

Paul Bremner and Ute Leonards. Speech and gesture emphasis effects for robotic and human communicators: a direct comparison. In Proceedings of the Tenth Annual ACM/IEEE International Conference on Human-Robot Interaction, pages 255-262. ACM, 2015.

Joost Broekens, Marcel Heerink, Henk Rosendal, et al. Assistive social robots in elderly care: a review. Gerontechnology, 8(2):94-103, 2009.

Douglas Brooks, Yu-ping Chen, and Ayanna M Howard. Simulation versus embodied agents: Does either induce better human adherence to physical therapy exercise? In 2012 4th IEEE RAS $\&$ EMBS International Conference on Biomedical Robotics and Biomechatronics (BioRob), pages 1715-1720. IEEE, 2012.

Rodney Brooks. Flesh and machines: How robots will change us. Vintage, 2002.

Rodney A Brooks. A robot that walks; emergent behaviors from a carefully evolved network. Neural computation, 1(2):253-262, 1989.

Rodney A Brooks. Elephants don't play chess. Robotics and autonomous systems, 6(1):3-15, 1990.

Lawrence J Brunner. Smiles can be back channels. Journal of Personality and Social Psychology, 37 (5):728, 1979.

Judee K Burgoon. Relational message interpretations of touch, conversational distance, and posture. Journal of Nonverbal behavior, 15(4):233-259, 1991.

Judee K Burgoon, Joseph A Bonito, Bjorn Bengtsson, Carl Cederberg, Magnus Lundeberg, and L Allspach. Interactivity in human-computer interaction: A study of credibility, understanding, and influence. Computers in human behavior, 16(6):553-574, 2000.

Lindsey Jacquelyn Byom and Bilge Mutlu. Theory of mind: Mechanisms, methods, and new directions. Frontiers in human neuroscience, 7:413, 2013.

Andrew J Calder, Andrew D Lawrence, Jill Keane, Sophie K Scott, Adrian M Owen, Ingrid Christoffels, and Andrew W Young. Reading the mind from eye gaze. Neuropsychologia, 40(8): 1129-1138, 2002.

David F Caldwell and Charles A O'Reilly III. Responses to failure: The effects of choice and responsibility on impression management. Academy of management journal, 25(1):121-136, 1982.

Gian Vittorio Caprara, Claudio Barbaranelli, Laura Borgogni, and Marco Perugini. The 'big five questionnaire': A new questionnaire to assess the five factor model. Personality and individual Differences, 15(3):281-288, 1993.

Justine Cassell. Embodied conversational agents: representation and intelligence in user interfaces. AI magazine, 22(4):67, 2001. 
Justine Cassell, Timothy Bickmore, Mark Billinghurst, Lee Campbell, Kenny Chang, Hannes Vilhjálmsson, and Hao Yan. Embodiment in conversational interfaces: Rea. In Proceedings of the SIGCHI conference on Human Factors in Computing Systems, pages 520-527. ACM, 1999.

Jyh-Jong Chang, Tung-I Wu, Wen-Lan Wu, and Fong-Chin Su. Kinematical measure for spastic reaching in children with cerebral palsy. Clinical Biomechanics, 20(4):381-388, 2005.

Mathieu Chollet, Kalin Stefanov, Helmut Prendinger, and Stefan Scherer. Public speaking training with a multimodal interactive virtual audience framework. In Proceedings of the 2015 ACM on International Conference on Multimodal Interaction, pages 367-368. ACM, 2015.

Caitlyn Clabaugh, Gisele Ragusa, Fei Sha, and Maja Matarić. Designing a socially assistive robot for personalized number concepts learning in preschool children. In 2015 Joint IEEE International Conference on Development and Learning and Epigenetic Robotics (ICDL-EpiRob), pages 314-319. IEEE, 2015.

Andy Clark. Re-inventing ourselves: The plasticity of embodiment, sensing, and mind. Journal of Medicine and Philosophy, 32(3):263-282, 2007.

Andy Clark. Supersizing the mind: Embodiment, action, and cognitive extension. OUP USA, 2008.

Brian Coltin and Manuela Veloso. Online pickup and delivery planning with transfers for mobile robots. In 2014 IEEE International Conference on Robotics and Automation (ICRA), pages 5786-5791. IEEE, 2014.

Sandra Costa, Alberto Brunete, Byung-Chull Bae, and Nikolaos Mavridis. Emotional storytelling using virtual and robotic agents. arXiv preprint arXiv:160\%.0532\%, 2016.

Paul C Cozby and Scott Bates. Methods in behavioral research. McGraw-Hill Education, 13th edition edition, 2017.

Rosa M Crum, James C Anthony, Susan S Bassett, and Marshal F Folstein. Population-based norms for the mini-mental state examination by age and educational level. Jama, 269(18):2386-2391, 1993.

Thomas J Csordas. Embodiment as a paradigm for anthropology. Ethos, 18(1):5-47, 1990.

Thomas J Csordas. Embodiment and experience: The existential ground of culture and self, volume 2. Cambridge University Press, 1994.

Antonio R Damasio. The feeling of what happens: Body and emotion in the making of consciousness. Houghton Mifflin Harcourt, 1999.

Kerstin Dautenhahn. I could be you: The phenomenological dimension of social understanding. Cybernetics \& Systems, 28(5):417-453, 1997.

Kerstin Dautenhahn. Editorial-socially intelligent agents-the human in the loop. IEEE Transactions on Systems, Man, and Cybernetics-Part A: Systems and Humans, 31(5):345-348, 2001.

Kerstin Dautenhahn. Design spaces and niche spaces of believable social robots. In Robot and Human Interactive Communication, 2002. Proceedings. 11th IEEE International Workshop on, pages 192-197. IEEE, 2002.

Eric C. Deng and Maja J. Matarić. Mime-inspired behaviors in minimal social robots. In 2017 ACM CHI Conference on Human Factors in Computing Systems Workshop on What Can Actors Teach Robots, May 2017. 
Eric C. Deng and Maja J. Matarić. Object-based generative methods for embodied gestures in socially interactive robots. In AAAI Spring Symposium on Designing the User Experience of Artificial Intelligence, Mar 2018.

Eric C Deng, Bilge Mutlu, and Maja J Matarić. Formalizing the design space and product development cycle for socially interactive robots. Workshop on Social Robots in the Wild at the 2018 ACM Conference on Human-Robot Interaction (HRI), 2018.

David DeVault, Ron Artstein, Grace Benn, Teresa Dey, Ed Fast, Alesia Gainer, Kallirroi Georgila, Jon Gratch, Arno Hartholt, Margaux Lhommet, et al. Simsensei kiosk: A virtual human interviewer for healthcare decision support. In Proceedings of the 2014 international conference on Autonomous agents and multi-agent systems, pages 1061-1068. International Foundation for Autonomous Agents and Multiagent Systems, 2014.

Thomas J Donahue and Matthias Scheutz. Investigating the effects of robot affect and embodiment on attention and natural language of human teammates. In Cognitive Infocommunications (CogInfoCom), 2015 6th IEEE International Conference on, pages 397-402. IEEE, 2015.

Brian R Duffy and Gina Joue. Intelligent robots: The question of embodiment. In Proc. of the Brain-Machine Workshop, 2000.

Starkey Duncan. Some signals and rules for taking speaking turns in conversations. Journal of personality and social psychology, 23(2):283, 1972.

Nat Durlach and Mel Slater. Presence in shared virtual environments and virtual togetherness. Presence: Teleoperators and Virtual Environments, 9(2):214-217, 2000.

Clive L Dym, Alice M Agogino, Ozgur Eris, Daniel D Frey, and Larry J Leifer. Engineering design thinking, teaching, and learning. Journal of Engineering Education, 94(1):103-120, 2005.

Gerald M Edelman. Wider than the sky: The phenomenal gift of consciousness. Yale University Press, 2004.

Amy C Edmondson and Stacy E McManus. Methodological fit in management field research. Academy of management review, 32(4):1246-1264, 2007.

Paul Ekman and Wallace V Friesen. Pictures of facial affect. consulting psychologists press, 1975.

David J. Feil-Seifer Elaine S. Short, Eric C. Deng and Maja J. Matarić. Understanding agency in interactions between children with autism and socially assistive robots. Transactions on Human-Robot Interaction, Dec 2017.

Nathan J Emery. The eyes have it: the neuroethology, function and evolution of social gaze. Neuroscience $\& 3$ Biobehavioral Reviews, 24(6):581-604, 2000.

Juan Fasola and Maja Mataric. A socially assistive robot exercise coach for the elderly. Journal of Human-Robot Interaction, 2(2):3-32, 2013.

David Feil-Seifer and Maja J Mataric. Defining socially assistive robotics. In 9th International Conference on Rehabilitation Robotics, 2005. ICORR 2005., pages 465-468. IEEE, 2005.

James Fieser and Bradley Dowden. Internet encyclopedia of philosophy. 2011.

Kerstin Fischer, Katrin Lohan, and Kilian Foth. Levels of embodiment: Linguistic analyses of factors influencing hri. In Human-Robot Interaction (HRI), 2012 7th ACM/IEEE International Conference on, pages 463-470. IEEE, 2012. 
Terrence Fong, Illah Nourbakhsh, and Kerstin Dautenhahn. A survey of socially interactive robots. Robotics and autonomous systems, 42(3):143-166, 2003.

Jodi Forlizzi and Carl DiSalvo. Service robots in the domestic environment: a study of the roomba vacuum in the home. In Proceedings of the 1st ACM SIGCHI/SIGART conference on Human-robot interaction, pages 258-265. ACM, 2006.

Leslie J Francis, Laurence B Brown, and Ronald Philipchalk. The development of an abbreviated form of the revised eysenck personality questionnaire (epqr-a): Its use among students in england, canada, the usa and australia. Personality and individual differences, 13(4):443-449, 1992.

Marina Fridin and Mark Belokopytov. Embodied robot versus virtual agent: Involvement of preschool children in motor task performance. International Journal of Human-Computer Interaction, 30 (6):459-469, 2014.

Alexandra Frischen, Andrew P Bayliss, and Steven P Tipper. Gaze cueing of attention: visual attention, social cognition, and individual differences. Psychological bulletin, 133(4):694, 2007.

King Sun Fu, Ralph Gonzalez, and CS George Lee. Robotics: Control Sensing. Vis. Tata McGraw-Hill Education, 1987.

J Furusho and M Masubuchi. Control of a dynamical biped locomotion system for steady walking. Journal of Dynamic Systems, Measurement, and Control, 108(2):111-118, 1986.

Alberto Gallace and Charles Spence. The science of interpersonal touch: an overview. Neuroscience E Biobehavioral Reviews, 34(2):246-259, 2010.

Maia Garau, Mel Slater, David-Paul Pertaub, and Sharif Razzaque. The responses of people to virtual humans in an immersive virtual environment. Presence: Teleoperators and Virtual Environments, 14(1):104-116, 2005.

Andre Gaschler, Sören Jentzsch, Manuel Giuliani, Kerstin Huth, Jan de Ruiter, and Alois Knoll. Social behavior recognition using body posture and head pose for human-robot interaction. In Intelligent Robots and Systems (IROS), 2012 IEEE/RSJ International Conference on, pages 2128-2133. IEEE, 2012.

Eleanor J Gibson. The concept of affordances in development: The renascence of functionalism. In The concept of development: The Minnesota symposia on child psychology, volume 15, pages 55-81. Lawrence Erlbaum Hillsdale, NJ, 1982.

Jennifer Goetz, Sara Kiesler, and Aaron Powers. Matching robot appearance and behavior to tasks to improve human-robot cooperation. In Robot and Human Interactive Communication, 2003. Proceedings. ROMAN 2003. The 12th IEEE International Workshop on, pages 55-60. Ieee, 2003.

Erving Goffman. The presentation of self in. Butler, Bodies that Matter, 1959.

Michael A Goodrich and Alan C Schultz. Human-robot interaction: a survey. Foundations and trends in human-computer interaction, 1(3):203-275, 2007.

Charles Goodwin. Action and embodiment within situated human interaction. Journal of pragmatics, 32(10):1489-1522, 2000.

Goren Gordon, Cynthia Breazeal, and Susan Engel. Can children catch curiosity from a social robot? In Proceedings of the Tenth Annual ACM/IEEE International Conference on Human-Robot Interaction, pages 91-98. ACM, 2015. 
Mark R Gover. The embodied mind: Cognitive science and human experience (book). Mind, Culture, and Activity, 3(4):295-299, 1996.

Jonathan Gratch, Susan Hill, Louis-Philippe Morency, David Pynadath, and David Traum. Exploring the implications of virtual human research for human-robot teams. In International Conference on Virtual, Augmented and Mixed Reality, pages 186-196. Springer, 2015.

Jillian Greczek, Elaine Short, Caitlyn E Clabaugh, Katelyn Swift-Spong, and Maja Mataric. Socially assistive robotics for personalized education for children. In AAAI Fall Symposium on Artificial Intelligence and Human-Robot Interaction (AI-HRI), 2014.

Charlotte N Gunawardena. Social presence theory and implications for interaction and collaborative learning in computer conferences. International journal of educational telecommunications, 1(2): 147-166, 1995.

Charlotte N Gunawardena and Frank J Zittle. Social presence as a predictor of satisfaction within a computer-mediated conferencing environment. American journal of distance education, 11(3): 8-26, 1997.

Sami Haddadin, Alin Albu-Schäffer, and Gerd Hirzinger. Requirements for safe robots: Measurements, analysis and new insights. The International Journal of Robotics Research, 28(11-12):1507-1527, 2009.

Edward T Hall. A system for the notation of proxemic behavior. American anthropologist, 65(5): 1003-1026, 1963.

Joy E Hanna and Susan E Brennan. Speakers' eye gaze disambiguates referring expressions early during face-to-face conversation. Journal of Memory and Language, 57(4):596-615, 2007.

Sandra G Hart. Nasa-task load index (nasa-tlx); 20 years later. In Proceedings of the human factors and ergonomics society annual meeting, volume 50, pages 904-908. Sage Publications Sage CA: Los Angeles, CA, 2006.

Catharina A Hartman, Ellen Luteijn, Marike Serra, and Ruud Minderaa. Refinement of the children's social behavior questionnaire (csbq): an instrument that describes the diverse problems seen in milder forms of pdd. Journal of Autism and Developmental Disorders, 36(3):325-342, 2006.

Dai Hasegawa, Justine Cassell, and Kenji Araki. The role of embodiment and perspective in direction-giving systems. In AAAI Fall Symposium: Dialog with Robots, 2010.

Kotaro Hayashi, Daisuke Sakamoto, Takayuki Kanda, Masahiro Shiomi, Satoshi Koizumi, Hiroshi Ishiguro, Tsukasa Ogasawara, and Norihiro Hagita. Humanoid robots as a passive-social medium-a field experiment at a train station. In Human-Robot Interaction (HRI), 2007 2nd ACM/IEEE International Conference on, pages 137-144. IEEE, 2007.

Leslie A Hayduk and Steven Mainprize. Personal space of the blind. Social Psychology Quarterly, pages 216-223, 1980.

Marcel Heerink, Ben Kröse, Vanessa Evers, and Bob Wielinga. Assessing acceptance of assistive social agent technology by older adults: the almere model. International journal of social robotics, 2(4):361-375, 2010.

Martin Heidegger. Art and space. Man and World, 6(1):3-8, 1973. 
Horst Hendriks-Jansen. Catching ourselves in the act: Situated activity, interactive emergence, evolution, and human thought. MIT Press, 1996.

Jari K Hietanen. Does your gaze direction and head orientation shift my visual attention? Neuroreport, 10(16):3443-3447, 1999.

Ken Hillis. Digital sensations: Space, identity, and embodiment in virtual reality. U of Minnesota Press, 1999.

Pamela J Hinds, Teresa L Roberts, and Hank Jones. Whose job is it anyway? a study of human-robot interaction in a collaborative task. Human-Computer Interaction, 19(1):151-181, 2004.

Timothy R Hinkin. A brief tutorial on the development of measures for use in survey questionnaires. Organizational research methods, 1(1):104-121, 1998.

Laura Hoffmann and Nicole C Krämer. Investigating the effects of physical and virtual embodiment in task-oriented and conversational contexts. International Journal of Human-Computer Studies, 71(7):763-774, 2013.

Thomas Holz, Mauro Dragone, and Gregory MP O'Hare. Where robots and virtual agents meet. International Journal of Social Robotics, 1(1):83-93, 2009.

J Hoonhout. Development of a rating scale to determine the enjoyability of user interactions with consumer devices. Technical report, Technical report, Philips Research, 2002.

Chien-Ming Huang and Bilge Mutlu. Robot behavior toolkit: generating effective social behaviors for robots. In Proceedings of the seventh annual ACM/IEEE international conference on Human-Robot Interaction, pages 25-32. ACM, 2012.

Chien-Ming Huang and Bilge Mutlu. Modeling and evaluating narrative gestures for humanlike robots. In Robotics: Science and Systems, pages 57-64, 2013.

Chien-Ming Huang and Bilge Mutlu. Learning-based modeling of multimodal behaviors for humanlike robots. In Proceedings of the 2014 ACM/IEEE international conference on Human-robot interaction, pages 57-64. ACM, 2014.

Chien-Ming Huang and Bilge Mutlu. Anticipatory robot control for efficient human-robot collaboration. In The Eleventh ACM/IEEE International Conference on Human Robot Interaction, pages 83-90. IEEE Press, 2016.

Chien-Ming Huang, Sean Andrist, Allison Sauppé, and Bilge Mutlu. Using gaze patterns to predict task intent in collaboration. Frontiers in psychology, 6:1049, 2015a.

Chien-Ming Huang, Maya Cakmak, and Bilge Mutlu. Adaptive coordination strategies for humanrobot handovers. In Robotics: Science and Systems, 2015b.

Kaoru Inoue, Kazuyoshi Wada, and Yuko Ito. Effective application of paro: Seal type robots for disabled people in according to ideas of occupational therapists. In International Conference on Computers for Handicapped Persons, pages 1321-1324. Springer, 2008.

Susan E Jackson and Randall S Schuler. A meta-analysis and conceptual critique of research on role ambiguity and role conflict in work settings. Organizational behavior and human decision processes, 36(1):16-78, 1985.

Joseph L Jones. Robots at the tipping point: the road to irobot roomba. IEEE Robotics 83 Automation Magazine, 13(1):76-78, 2006. 
Stanley E Jones and A Elaine Yarbrough. A naturalistic study of the meanings of touch. Communications Monographs, 52(1):19-56, 1985.

Céline Jost, Vanessa André, Brigitte Le Pévédic, Alban Lemasson, Martine Hausberger, and Dominique Duhaut. Ethological evaluation of human-robot interaction: are children more efficient and motivated with computer, virtual agent or robots? In Robotics and Biomimetics (ROBIO), 2012 IEEE International Conference on, pages 1368-1373. IEEE, 2012a.

Céline Jost, Brigitte Le Pévédic, and Dominique Duhaut. Robot is best to play with human! In $R O$ MAN 2012-21st IEEE International Symposium on Robot and Human Interactive Communication, 2012b.

Céline Jost, Marine Grandgeorge, Brigitte Le Pévédic, and Dominique Duhaut. Robot or tablet: Users' behaviors on a memory game. In Robot and Human Interactive Communication, 2014 RO-MAN: The 23rd IEEE International Symposium on, pages 1050-1055. IEEE, 2014.

Wendy Ju and David Sirkin. Animate objects: How physical motion encourages public interaction. In International Conference on Persuasive Technology, pages 40-51. Springer, 2010.

Merel M Jung, Mannes Poel, Dennis Reidsma, and Dirk KJ Heylen. a first step toward the automatic understanding of social touch for naturalistic human-robot interaction. Frontiers in ICT, 4:3, 2017.

Younbo Jung and Kwan Min Lee. Effects of physical embodiment on social presence of social robots. Proceedings of PRESENCE, pages 80-87, 2004.

Lee Jussim. Social perception and social reality: A reflection-construction model. Psychological review, 98(1):54, 1991.

Alisa Kalegina, Grace Schroeder, Aidan Allchin, Keara Berlin, and Maya Cakmak. Characterizing the design space of rendered robot faces. In Proceedings of the 2018 ACM/IEEE international conference on Human-robot interaction, 2018.

Takayuki Kanda, Takayuki Hirano, Daniel Eaton, and Hiroshi Ishiguro. Interactive robots as social partners and peer tutors for children: A field trial. Human-computer interaction, 19(1):61-84, 2004 .

Immanuel Kant and Stanley L Jaki. Universal natural history and theory of the heavens. Edinburgh: Scottish Academic Press, 1981., 1, 1981.

James Kennedy, Paul Baxter, and Tony Belpaeme. The robot who tried too hard: Social behaviour of a robot tutor can negatively affect child learning. In Proceedings of the Tenth Annual ACM/IEEE International Conference on Human-Robot Interaction, pages 67-74. ACM, 2015.

William G Kennedy, Magdalena D Bugajska, Matthew Marge, William Adams, Benjamin R Fransen, Dennis Perzanowski, Alan C Schultz, and J Gregory Trafton. Spatial representation and reasoning for human-robot collaboration. In $A A A I$, volume 7, pages 1554-1559, 2007.

Cory D Kidd and Cynthia Breazeal. Effect of a robot on user perceptions. In Intelligent Robots and Systems, 2004.(IROS 2004). Proceedings. 2004 IEEE/RSJ International Conference on, volume 4, pages 3559-3564. IEEE, 2004.

Sara Kiesler, Aaron Powers, Susan R Fussell, and Cristen Torrey. Anthropomorphic interactions with a robot and robot-like agent. Social Cognition, 26(2):169, 2008. 
Konstantina Kilteni, Raphaela Groten, and Mel Slater. The sense of embodiment in virtual reality. Presence: Teleoperators and Virtual Environments, 21(4):373-387, 2012.

Taeyong Kim and Frank Biocca. Telepresence via television: Two dimensions of telepresence may have different connections to memory and persuasion.[1]. Journal of Computer-Mediated Communication, 3(2):0-0, 1997.

Tineke Klamer, Somaya Ben Allouch, and Dirk Heylen. 'adventures of harvey'-use, acceptance of and relationship building with a social robot in a domestic environment. In International Conference on Human-Robot Personal Relationship, pages 74-82. Springer, 2010.

Lisa R Klein. Creating virtual product experiences: The role of telepresence. Journal of interactive Marketing, 17(1):41-55, 2003.

Mark L Knapp, Judith A Hall, and Terrence G Horgan. Nonverbal communication in human interaction. Cengage Learning, 2013.

Takanori Komatsu. Comparison an On-screen Agent with a Robotic Agent in an Everyday Interaction Style: How to Make Users React Toward an On-screen Agent as if They are Reacting Toward a Robotic Agent. INTECH Open Access Publisher, 2010.

Hatice Kose-Bagci, Ester Ferrari, Kerstin Dautenhahn, Dag Sverre Syrdal, and Chrystopher L Nehaniv. Effects of embodiment and gestures on social interaction in drumming games with a humanoid robot. Advanced Robotics, 23(14):1951-1996, 2009.

Nicole C Krämer. Social communicative effects of a virtual program guide. In International Workshop on Intelligent Virtual Agents, pages 442-453. Springer, 2005.

Robert M Krauss. Why do we gesture when we speak? Current Directions in Psychological Science, $7(2): 54-54,1998$.

Klaus Krippendorff. Reliability in content analysis. Human communication research, 30(3):411-433, 2004.

Anders Krogsager, Nicolaj Segato, and Matthias Rehm. Backchannel head nods in danish first meeting encounters with a humanoid robot: The role of physical embodiment. In International Conference on Human-Computer Interaction, pages 651-662. Springer, 2014.

Pauline A Langen, Jeffrey S Katz, Gayle Dempsey, and James Pompano. Remote monitoring of high-risk patients using artificial intelligence, October 18 1994. US Patent 5,357,427.

Przemyslaw A Lasota and Julie A Shah. Analyzing the effects of human-aware motion planning on close-proximity human-robot collaboration. Human factors, 57(1):21-33, 2015.

Jonathan Lazar, Jinjuan Heidi Feng, and Harry Hochheiser. Research methods in human-computer interaction. Morgan Kaufmann, 2017.

Jee Yoon Lee, Jung Ju Choi, and Sonya S Kwak. The impact of user control design types on people's perception of a robot. In Proceedings of the Tenth Annual ACM/IEEE International Conference on Human-Robot Interaction Extended Abstracts, pages 19-20. ACM, 2015.

KM Lee, N Park, and H Song. Can a robot be perceived as a developing creature?: Effects of artificial developments on social presence and social responses toward robots in human-robot interaction. In International Communication Association conference, 2004.

Kwan Min Lee. Presence, explicated. Communication theory, 14(1):27-50, 2004. 
Kwan Min Lee, Younbo Jung, Jaywoo Kim, and Sang Ryong Kim. Are physically embodied social agents better than disembodied social agents?: The effects of physical embodiment, tactile interaction, and people's loneliness in human-robot interaction. International Journal of HumanComputer Studies, 64(10):962-973, 2006.

Mark H Lee and Howard R Nicholls. Review article tactile sensing for mechatronics' state of the art survey. Mechatronics, 9(1):1-31, 1999.

Sau-lai Lee, Ivy Yee-man Lau, Sara Kiesler, and Chi-Yue Chiu. Human mental models of humanoid robots. In Robotics and Automation, 2005. ICRA 2005. Proceedings of the 2005 IEEE International Conference on, pages 2767-2772. IEEE, 2005.

Wan-Ju Lee, Chi-Wen Huang, Chia-Jung Wu, Shing-Tsaan Huang, and Gwo-Dong Chen. The effects of using embodied interactions to improve learning performance. In Advanced learning technologies (icalt), 2012 ieee 12th international conference on, pages 557-559. IEEE, 2012.

Iolanda Leite, André Pereira, Carlos Martinho, and Ana Paiva. Are emotional robots more fun to play with? In Robot and human interactive communication, 2008. RO-MAN 2008. The 17th IEEE international symposium on, pages 77-82. IEEE, 2008.

Jesse Levinson, Jake Askeland, Jan Becker, Jennifer Dolson, David Held, Soeren Kammel, J Zico Kolter, Dirk Langer, Oliver Pink, Vaughan Pratt, et al. Towards fully autonomous driving: Systems and algorithms. In Intelligent Vehicles Symposium (IV), 2011 IEEE, pages 163-168. IEEE, 2011.

Shelly Levy-Tzedek, Sigal Berman, Yehuda Stiefel, Ehud Sharlin, James Young, and Daniel Rea. Robotic mirror game for movement rehabilitation. In Virtual Rehabilitation (ICVR), 2017 International Conference on, pages 1-2. IEEE, 2017.

Daniel Leyzberg, Samuel Spaulding, Mariya Toneva, and Brian Scassellati. The physical presence of a robot tutor increases cognitive learning gains. 2012.

Jamy Li and Mark Chignell. Communication of emotion in social robots through simple head and arm movements. International Journal of Social Robotics, 3(2):125-142, 2011.

Mike Ligthart and Khiet P Truong. Selecting the right robot: Influence of user attitude, robot sociability and embodiment on user preferences. In Robot and Human Interactive Communication (RO-MAN), 2015 24th IEEE International Symposium on, pages 682-687. IEEE, 2015.

Rensis Likert. A technique for the measurement of attitudes. Archives of psychology, 1932.

Katrin Solveig Lohan, Sebastian Gieselmann, Anna-Lisa Vollmer, Katharina Rohlfing, and Britta Wrede. Does embodiment affect tutoring behavior. In IEEE international conference on development and learning (ICDL) conference, 2010.

Matthew Lombard and Theresa Ditton. At the heart of it all: The concept of presence. Journal of Computer-Mediated Communication, 3(2):0-0, 1997.

Matthew Lombard, Theresa B Ditton, Daliza Crane, Bill Davis, Gisela Gil-Egui, Karl Horvath, Jessica Rossman, and S Park. Measuring presence: A literature-based approach to the development of a standardized paper-and-pencil instrument. In Third international workshop on presence, delft, the netherlands, volume 240, pages 2-4, 2000.

Matthew R Longo, Friederike Schüür, Marjolein PM Kammers, Manos Tsakiris, and Patrick Haggard. What is embodiment? a psychometric approach. Cognition, 107(3):978-998, 2008. 
Rosemarijn Looije, Mark A Neerincx, and Fokie Cnossen. Persuasive robotic assistant for health self-management of older adults: Design and evaluation of social behaviors. International Journal of Human-Computer Studies, 68(6):386-397, 2010.

Rosemarijn Looije, Anna van der Zalm, Mark A Neerincx, and Robbert-Jan Beun. Help, I need some body the effect of embodiment on playful learning. IEEE, 2012.

K Louise Barriball and Alison While. Collecting data using a semi-structured interview: a discussion paper. Journal of advanced nursing, 19(2):328-335, 1994.

Joe C Magee and Adam D Galinsky. 8 social hierarchy: The self-reinforcing nature of power and status. Academy of Management annals, 2(1):351-398, 2008.

Jim Mainprice and Dmitry Berenson. Human-robot collaborative manipulation planning using early prediction of human motion. In 2013 IEEE/RSJ International Conference on Intelligent Robots and Systems, pages 299-306. IEEE, 2013.

Jeremy Maitin-Shepard, Marco Cusumano-Towner, Jinna Lei, and Pieter Abbeel. Cloth grasp point detection based on multiple-view geometric cues with application to robotic towel folding. In Robotics and Automation (ICRA), 2010 IEEE International Conference on, pages 2308-2315. IEEE, 2010.

Giuseppe Mantovani and Giuseppe Riva. "Real" presence: how different ontologies generate different criteria for presence, telepresence, and virtual presence. Presence: Teleoperators and Virtual Environments, 8(5):540-550, 1999.

Malia F Mason, Elizabeth P Tatkow, and C Neil Macrae. The look of love: Gaze shifts and person perception. Psychological Science, 16(3):236-239, 2005.

Matthew T Mason and J Kenneth Salisbury Jr. Robot hands and the mechanics of manipulation. 1985.

Bjoern Matthias, Soenke Kock, Henrik Jerregard, Mats Källman, and Ivan Lundberg. Safety of collaborative industrial robots: Certification possibilities for a collaborative assembly robot concept. In Assembly and Manufacturing (ISAM), 2011 IEEE International Symposium on, pages 1-6. IEEE, 2011.

Humberto R Maturana and Francisco J Varela. The tree of knowledge: The biological roots of human understanding. New Science Library/Shambhala Publications, 1987.

Humberto R Maturana and Francisco J Varela. Autopoiesis and cognition: The realization of the living, volume 42. Springer Science \& Business Media, 1991.

Evelyn Z McClave. Linguistic functions of head movements in the context of speech. Journal of pragmatics, 32(7):855-878, 2000.

James C McCroskey and Thomas A McCain. The measurement of interpersonal attraction. 1974.

E Mcgrath. Methodology matters: Doing research in the behavioral and social sciences. In Readings in Human-Computer Interaction: Toward the Year 2000 (2nd ed. Citeseer, 1995.

David McNeill. Gesture and thought. University of Chicago press, 2008.

N.A. McQuown and G. Bateson. The Natural History of an Interview. Microfilm collection of manuscripts on cultural anthropology, ser 15, no. 95-98. University of Chicago Library, 1971. 
Ross Mead and Maja J Matarić. Perceptual models of human-robot proxemics. In Experimental Robotics, pages 261-276. Springer, 2016.

Ross Mead and Maja J Matarić. Autonomous human-robot proxemics: socially aware navigation based on interaction potential. Autonomous Robots, 41(5):1189-1201, 2017.

Ross Mead, Amin Atrash, and Maja J Matarić. Automated proxemic feature extraction and behavior recognition: Applications in human-robot interaction. International Journal of Social Robotics, 5 (3):367-378, 2013.

Albert Mehrabian. Significance of posture and position in the communication of attitude and status relationships. Psychological Bulletin, 71(5):359, 1969.

Albert Mehrabian. Nonverbal communication. Transaction Publishers, 1972.

Zahira Merchant, Ernest T Goetz, Lauren Cifuentes, Wendy Keeney-Kennicutt, and Trina J Davis. Effectiveness of virtual reality-based instruction on students' learning outcomes in k-12 and higher education: A meta-analysis. Computers \& Education, 70:29-40, 2014.

Maurice Merleau-Ponty, Oliver Davis, and Thomas Baldwin. The world of perception. Cambridge Univ Press, 2004.

Ashley Montagu and Floyd W Matson. The human connection. McGraw-Hill, 1979.

Hans Moravec. Mind children, volume 375. Cambridge Univ Press, 1988a.

Hans P Moravec. Sensor fusion in certainty grids for mobile robots. AI magazine, 9(2):61, 1988b.

Masahiro Mori. The uncanny valley. Energy, 7(4):33-35, 1970.

Frederick Mosteller, Robert Ray Bush, and Bert Franklin Green. Selected quantitative techniques. Addison-Wesley, 1954.

Maria Moundridou and Maria Virvou. Evaluating the persona effect of an interface agent in a tutoring system. Journal of computer assisted learning, 18(3):253-261, 2002.

Emily Mower, Maja J Mataric, and Shrikanth Narayanan. Human perception of audio-visual synthetic character emotion expression in the presence of ambiguous and conflicting information. IEEE Transactions on Multimedia, 11(5):843-855, 2009.

Jonathan Mumm and Bilge Mutlu. Human-robot proxemics: physical and psychological distancing in human-robot interaction. In Proceedings of the 6th international conference on Human-robot interaction, pages 331-338. ACM, 2011.

Robin Murphy. Introduction to AI robotics. MIT press, 2000.

Bilge Mutlu. Designing embodied cues for dialog with robots. AI Magazine, 32(4):17-30, 2011.

Bilge Mutlu. Virtual and physical: Two frames of mind, 2017.

Bilge Mutlu and Jodi Forlizzi. Robots in organizations: the role of workflow, social, and environmental factors in human-robot interaction. In Human-Robot Interaction (HRI), 2008 3rd ACM/IEEE International Conference on, pages 287-294. IEEE, 2008.

Bilge Mutlu, Jodi Forlizzi, and Jessica Hodgins. A storytelling robot: Modeling and evaluation of human-like gaze behavior. In Humanoid robots, 2006 6th IEEE-RAS international conference on, pages 518-523. IEEE, 2006. 
Bilge Mutlu, Toshiyuki Shiwa, Takayuki Kanda, Hiroshi Ishiguro, and Norihiro Hagita. Footing in human-robot conversations: how robots might shape participant roles using gaze cues. In Proceedings of the 4th ACM/IEEE international conference on Human robot interaction, pages 61-68. ACM, 2009.

Bilge Mutlu, Takayuki Kanda, Jodi Forlizzi, Jessica Hodgins, and Hiroshi Ishiguro. Conversational gaze mechanisms for humanlike robots. ACM Transactions on Interactive Intelligent Systems (TiiS), 1(2):12, 2012.

John Nash. Non-cooperative games. Annals of mathematics, pages 286-295, 1951.

Stefanos Nikolaidis and Julie Shah. Human-robot cross-training: computational formulation, modeling and evaluation of a human team training strategy. In Proceedings of the 8th ACM/IEEE international conference on Human-robot interaction, pages 33-40. IEEE Press, 2013.

Stefanos Nikolaidis, Swaprava Nath, Ariel D Procaccia, and Siddhartha Srinivasa. Game-theoretic modeling of human adaptation in human-robot collaboration. In Proceedings of the 2017 ACM/IEEE International Conference on Human-Robot Interaction, pages 323-331. ACM, 2017.

Christos Nikolopoulos, Deitra Kuester, Mark Sheehan, Shashwati Ramteke, Aniket Karmarkar, Supriya Thota, Joseph Kearney, Curtis Boirum, Sunnihith Bojedla, and Angela Lee. Robotic agents used to help teach social skills to children with autism: the third generation. In 2011 RO-MAN, pages 253-258. IEEE, 2011.

L PJJ Noldus. The observer: a software system for collection and analysis of observational data. Behavior Research Methods, 23(3):415-429, 1991.

Tatsuya Nomura and Miyuki Sasa. Investigation of differences on impressions of and behaviors toward real and virtual robots between elder people and university students. In Rehabilitation Robotics, 2009. ICORR 2009. IEEE International Conference on, pages 934-939. IEEE, 2009.

Tatsuya Nomura, Tomohiro Suzuki, Takayuki Kanda, and Kensuke Kato. Altered attitudes of people toward robots: Investigation through the negative attitudes toward robots scale. In Proc. AAAI-06 Workshop on Human Implications of Human-Robot Interaction, volume 2006, pages 29-35, 2006.

Donald A Norman. Affordance, conventions, and design. interactions, 6(3):38-43, 1999.

Illah R Nourbakhsh, Judith Bobenage, Sebastien Grange, Ron Lutz, Roland Meyer, and Alvaro Soto. An affective mobile robot educator with a full-time job. Artificial Intelligence, 114(1-2): 95-124, 1999.

Judith S Olson and Wendy A Kellogg. Ways of Knowing in HCI, volume 2. Springer, 2014.

Tetsuo Ono, Michita Imai, and Hiroshi Ishiguro. A model of embodied communications with gestures between humans and robots. In Proceedings of 23rd annual meeting of the cognitive science society, pages 732-737. Citeseer, 2001.

Ortega and José Gasset. Vitalidad, alma, espíritu. Cuerpo vivido, pages 15-52, 2010.

Fumiaki Osawa, Hiroaki Seki, and Yoshitsugu Kamiya. Clothes folding task by tool-using robot. Journal of Robotics and Mechatronics, 18(5):618, 2006.

Don R Osborn. Beauty is as beauty does?: Makeup and posture effects on physical attractiveness judgments. Journal of Applied Social Psychology, 26(1):31-51, 1996. 
Emma Otta, Beatriz Barcellos Pereira Lira, Nadia Maria Delevati, Otávio Pimentel Cesar, and Carla Salati Guirello Pires. The effect of smiling and of head tilting on person perception. The Journal of psychology, 128(3):323-331, 1994.

Ye Pan and Anthony Steed. a comparison of avatar-, video-, and robot-mediated interaction on users' trust in expertise. Frontiers in Robotics and AI, 3:12, 2016.

Richard H Passman and Paul Weisberg. Mothers and blankets as agents for promoting play and exploration by young children in a novel environment: The effects of social and nonsocial attachment objects. Developmental Psychology, 11(2):170, 1975.

Margaret Pearce, Bilge Mutlu, Julie Shah, and Robert Radwin. Optimizing makespan and ergonomics in integrating collaborative robots into manufacturing processes. IEEE Transactions on Automation Science and Engineering, 2018.

Tomislav Pejsa, Sean Andrist, Michael Gleicher, and Bilge Mutlu. Gaze and attention management for embodied conversational agents. ACM Transactions on Interactive Intelligent Systems (TiiS), $5(1): 3,2015$.

Julia Peltason, Nina Riether, Britta Wrede, and Ingo Lütkebohle. Talking with robots about objects: a system-level evaluation in hri. In Human-Robot Interaction (HRI), 2012 7th ACM/IEEE International Conference on, pages 479-486. IEEE, 2012.

André Pereira, Carlos Martinho, Iolanda Leite, and Ana Paiva. icat, the chess player: the influence of embodiment in the enjoyment of a game. In Proceedings of the 7th international joint conference on Autonomous agents and multiagent systems-Volume 3, pages 1253-1256. International Foundation for Autonomous Agents and Multiagent Systems, 2008.

Ken Perlin and Athomas Goldberg. Improv: A system for scripting interactive actors in virtual worlds. In Proceedings of the 23rd annual conference on Computer graphics and interactive techniques, pages 205-216. ACM, 1996.

Per Persson, Jarmo Laaksolahti, and P Lonnqvist. Understanding socially intelligent agentsa multilayered phenomenon. IEEE Transactions on Systems, Man, and Cybernetics-Part A: Systems and Humans, 31(5):349-360, 2001.

Rolf Pfeifer and Christian Scheier. Understanding intelligence. MIT press, 2001.

Jonathan Posner, James A Russell, and Bradley S Peterson. The circumplex model of affect: An integrative approach to affective neuroscience, cognitive development, and psychopathology. Development and psychopathology, 17(3):715-734, 2005.

Aaron Powers and Sara Kiesler. The advisor robot: tracing people's mental model from a robot's physical attributes. In Proceedings of the 1st ACM SIGCHI/SIGART conference on Human-robot interaction, pages 218-225. ACM, 2006.

Aaron Powers, Sara Kiesler, Susan Fussell, and Cristen Torrey. Comparing a computer agent with a humanoid robot. In Human-Robot Interaction (HRI), 2007 2nd ACM/IEEE International Conference on, pages 145-152. IEEE, 2007.

Paul C. Price, Rajiv S. Jhangiani, I-Chant A. Chiang, Dana C. Leighton, and Carrie Cuttler. Research Methods in Psychology. Open Textbook Library, 3rd american edition edition, 2017. 
Tom Quick, Kerstin Dautenhahn, Chrystopher L Nehaniv, and Graham Roberts. On bots and bacteria: Ontology independent embodiment. In European Conference on Artificial Life, pages 339-343. Springer, 1999.

Pramila Rani, Nilanjan Sarkar, Craig A Smith, and Leslie D Kirby. Anxiety detecting robotic system-towards implicit human-robot collaboration. Robotica, 22(01):85-95, 2004.

Fredrik Rehnmark, William Bluethmann, Joshua Mehling, Robert O Ambrose, Myron Diftler, Mars Chu, and Ryan Necessary. Robonaut: the'short list'of technology hurdles. Computer, 38(1):28-37, 2005 .

W Scott Reilly. Believable social and emotional agents. Technical report, DTIC Document, 1996.

Nadine Richard, Philippe Codognet, and Alain Grumbach. The inviwo toolkit: Describing autonomous virtual agents and avatars. In International Workshop on Intelligent Virtual Agents, pages 195-209. Springer, 2001.

Raoul Rickenberg and Byron Reeves. The effects of animated characters on anxiety, task performance, and evaluations of user interfaces. In Proceedings of the SIGCHI conference on Human Factors in Computing Systems, pages 49-56. ACM, 2000.

Alexander Riegler. When is a cognitive system embodied? Cognitive Systems Research, 3(3):339-348, 2002.

Eugene I Rivin. Mechanical design of robots. McGraw-Hill, Inc., 1987.

Albert Rizzo, JoAnn Difede, Barbara O Rothbaum, Greg Reger, Josh Spitalnick, Judith Cukor, Rob Mclay, et al. Development and early evaluation of the virtual iraq/afghanistan exposure therapy system for combat-related ptsd. Annals of the New York Academy of Sciences, 1208(1):114-125, 2010.

Ben Robins, Kerstin Dautenhahn, and Janek Dubowski. Does appearance matter in the interaction of children with autism with a humanoid robot? Interaction Studies, 7(3):509-542, 2006.

Eleanor Rosch, Francisco Varela, and Evan Thompson. The embodied mind. Cognitive Science and Human Experience, 1991.

Ulrich Rothbauer, Kourosh Zolghadr, Serge Muyldermans, Aloys Schepers, M Cristina Cardoso, and Heinrich Leonhardt. A versatile nanotrap for biochemical and functional studies with fluorescent fusion proteins. Molecular \& Cellular Proteomics, 7(2):282-289, 2008.

Kerstin Ruhland, Christopher E Peters, Sean Andrist, Jeremy B Badler, Norman I Badler, Michael Gleicher, Bilge Mutlu, and Rachel McDonnell. A review of eye gaze in virtual agents, social robotics and hci: Behaviour generation, user interaction and perception. In Computer Graphics Forum, volume 34, pages 299-326. Wiley Online Library, 2015.

Daniel W Russell. Ucla loneliness scale (version 3): Reliability, validity, and factor structure. Journal of personality assessment, 66(1):20-40, 1996.

James A Russell, Jo-Anne Bachorowski, and José-Miguel Fernández-Dols. Facial and vocal expressions of emotion. Annual review of psychology, 54(1):329-349, 2003.

Martin Saerbeck, Tom Schut, Christoph Bartneck, and Maddy D Janse. Expressive robots in education: varying the degree of social supportive behavior of a robotic tutor. In Proceedings of the SIGCHI Conference on Human Factors in Computing Systems, pages 1613-1622. ACM, 2010. 
Gustavo Saposnik, Mindy Levin, Stroke Outcome Research Canada (SORCan) Working Group, et al. Virtual reality in stroke rehabilitation a meta-analysis and implications for clinicians. Stroke, 42 (5):1380-1386, 2011.

Wataru Sato, Takanori Kochiyama, Shota Uono, and Sakiko Yoshikawa. Commonalities in the neural mechanisms underlying automatic attentional shifts by gaze, gestures, and symbols. Neuroimage, 45(3):984-992, 2009.

Stefan Scherer, Stacy Marsella, Giota Stratou, Yuyu Xu, Fabrizio Morbini, Alesia Egan, Albert Rizzo, and Louis-Philippe Morency. Perception markup language: Towards a standardized representation of perceived nonverbal behaviors. In Intelligent virtual agents, pages 455-463. Springer, 2012.

Elena Márquez Segura, Michael Kriegel, Ruth Aylett, Amol Deshmukh, and Henriette Cramer. How do you like me in this: User embodiment preferences for companion agents. In International Conference on Intelligent Virtual Agents, pages 112-125. Springer, 2012.

Suleman Shahid, Emiel Krahmer, and Marc Swerts. Child-robot interaction across cultures: How does playing a game with a social robot compare to playing a game alone or with a friend? Computers in Human Behavior, 40:86-100, 2014.

Lawrence Shapiro. Embodied cognition. Routledge, 2010.

Noel E Sharkey and Tom Ziemke. Mechanistic versus phenomenal embodiment: Can robot embodiment lead to strong ai? Cognitive Systems Research, 2(4):251-262, 2001.

Kuniya Shinozaki, Akitsugu Iwatani, and Ryohei Nakatsu. Construction and evaluation of a robot dance system. In New Frontiers for Entertainment Computing, pages 83-94. Springer, 2008.

Kazuhiko Shinozawa and Junji Yamato. Effect of Robot and Screen Agent Recommendations on Human Decision-Making. Citeseer, 2007.

Kazuhiko Shinozawa, Byron Reeves, Kevin Wise, Sohye Lim, Heidy Maldonado, and Futoshi Naya. Robots as new media: A cross-cultural examination of social and cognitive responses to robotic and on-screen agents. In Proceedings of Annual Conference of Internation Communication Association, pages 998-1002, 2003.

Candace L Sidner, Christopher Lee, Cory D Kidd, Neal Lesh, and Charles Rich. Explorations in engagement for humans and robots. Artificial Intelligence, 166(1):140-164, 2005.

Reid Simmons, Richard Goodwin, Karen Zita Haigh, Sven Koenig, and Joseph O'Sullivan. A layered architecture for office delivery robots. In Proceedings of the first international conference on Autonomous agents, pages 245-252. ACM, 1997.

Reid Simmons, Sanjiv Singh, David Hershberger, Josue Ramos, and Trey Smith. First results in the coordination of heterogeneous robots for large-scale assembly. In Experimental Robotics VII, pages 323-332. Springer, 2001.

Shamus P Smith and Michael D Harrison. Editorial: User centred design and implementation of virtual environments. International journal of human-computer studies, 55(2):109-114, 2001.

James G Snider and Charles Egerton Osgood. Semantic differential technique; a sourcebook. Aldine Pub. Co., 1969.

Marc Stickdorn, Jakob Schneider, Kate Andrews, and Adam Lawrence. This is service design thinking: Basics, tools, cases. Wiley Hoboken, NJ, 2011. 
Kyle Wayne Strabala, Min Kyung Lee, Anca Diana Dragan, Jodi Lee Forlizzi, Siddhartha Srinivasa, Maya Cakmak, and Vincenzo Micelli. Towards seamless human-robot handovers. Journal of Human-Robot Interaction, 2(1):112-132, 2013.

Anselm Strauss and Juliet M Corbin. Grounded theory in practice. Sage, 1997.

John Sweller. Cognitive load during problem solving: Effects on learning. Cognitive science, 12(2): 257-285, 1988.

Leila Takayama and Caroline Pantofaru. Influences on proxemic behaviors in human-robot interaction. In 2009 IEEE/RSJ International Conference on Intelligent Robots and Systems, pages 5495-5502. IEEE, 2009.

Johane Takeuchi, Kazutaka Kushida, Yoshitaka Nishimura, Hiroshi Dohi, Mitsuru Ishizuka, Mikio Nakano, and Hiroshi Tsujino. Comparison of a humanoid robot and an on-screen agent as presenters to audiences. In 2006 IEEE/RSJ International Conference on Intelligent Robots and Systems, pages 3964-3969. IEEE, 2006.

Adriana Tapus, Cristian Tapus, and Maja Mataric. The role of physical embodiment of a therapist robot for individuals with cognitive impairments. In RO-MAN 2009-The 18th IEEE International Symposium on Robot and Human Interactive Communication, pages 103-107. IEEE, 2009.

Tina L Taylor. Living digitally: Embodiment in virtual worlds. In The social life of avatars, pages 40-62. Springer, 2002.

Michael Tomasello. Origins of human communication. MIT press, 2010.

Tarik Tosun, Ross Mead, and Robert Stengel. A general method for kinematic retargeting: Adapting poses between humans and robots. In ASME 2014 international mechanical engineering congress and exposition, pages V04AT04A027-V04AT04A027. American Society of Mechanical Engineers, 2014.

David Traum, Stacy C Marsella, Jonathan Gratch, Jina Lee, and Arno Hartholt. Multi-party, multi-issue, multi-strategy negotiation for multi-modal virtual agents. In International Workshop on Intelligent Virtual Agents, pages 117-130. Springer, 2008.

Fransisco J Valera, Evan Thompson, and Eleanor Rosch. The embodied mind. Cognitive Science and Human Experience, 1991.

Viswanath Venkatesh, Michael G Morris, Gordon B Davis, and Fred D Davis. User acceptance of information technology: Toward a unified view. MIS quarterly, pages 425-478, 2003.

Vinoba Vinayagamoorthy, Maia Garau, Anthony Steed, and Mel Slater. An eye gaze model for dyadic interaction in an immersive virtual environment: Practice and experience. In Computer Graphics Forum, volume 23, pages 1-11. Wiley Online Library, 2004.

Spyros Vosinakis and Themis Panayiotopoulos. Simhuman: A platform for real-time virtual agents with planning capabilities. In International Workshop on Intelligent Virtual Agents, pages 210-223. Springer, 2001.

Suzanne Vossen, Jaap Ham, and Cees Midden. Social influence of a persuasive agent: the role of agent embodiment and evaluative feedback. In Proceedings of the 4 th International Conference on Persuasive Technology, page 46. ACM, 2009. 
Kazuyoshi Wada and Takanori Shibata. Robot therapy in a care house-its sociopsychological and physiological effects on the residents. In Proceedings 2006 IEEE International Conference on Robotics and Automation, 2006. ICRA 2006., pages 3966-3971. IEEE, 2006.

Daniel Wagner, Mark Billinghurst, and Dieter Schmalstieg. How real should virtual characters be? In Proceedings of the 2006 ACM SIGCHI international conference on Advances in computer entertainment technology, page 57. ACM, 2006.

Joshua Wainer, David J Feil-Seifer, Dylan A Shell, and Maja J Mataric. The role of physical embodiment in human-robot interaction. In ROMAN 2006-The 15th IEEE International Symposium on Robot and Human Interactive Communication, pages 117-122. IEEE, 2006.

Joshua Wainer, David J Feil-Seifer, Dylan A Shell, and Maja J Mataric. Embodiment and humanrobot interaction: A task-based perspective. In RO-MAN 2007-The 16th IEEE International Symposium on Robot and Human Interactive Communication, pages 872-877. IEEE, 2007.

Vincent R Waldron. Achieving communication goals in superior-subordinate relationships: The multi-functionality of upward maintenance tactics. Communications Monographs, 58(3):289-306, 1991.

Michael L Walters, Kerstin Dautenhahn, René Te Boekhorst, Kheng Lee Koay, Christina Kaouri, Sarah Woods, Chrystopher Nehaniv, David Lee, and Iain Werry. The influence of subjects' personality traits on personal spatial zones in a human-robot interaction experiment. In ROMAN 2005. IEEE International Workshop on Robot and Human Interactive Communication, 2005., pages 347-352. IEEE, 2005.

A Ting Wang, Susan S Lee, Marian Sigman, and Mirella Dapretto. Developmental changes in the neural basis of interpreting communicative intent. Social cognitive and affective neuroscience, 1 (2):107-121, 2006.

Chieh-Chih Wang and Chuck Thorpe. Simultaneous localization and mapping with detection and tracking of moving objects. In Robotics and Automation, 2002. Proceedings. ICRA'02. IEEE International Conference on, volume 3, pages 2918-2924. IEEE, 2002.

David Watson, Lee Anna Clark, and Auke Tellegen. Development and validation of brief measures of positive and negative affect: the panas scales. Journal of personality and social psychology, 54 (6):1063, 1988.

Robert B Welch, Theodore T Blackmon, Andrew Liu, Barbara A Mellers, and Lawrence W Stark. The effects of pictorial realism, delay of visual feedback, and observer interactivity on the subjective sense of presence. Presence: Teleoperators \& Virtual Environments, 5(3):263-273, 1996.

Iain Werry, Kerstin Dautenhahn, Bernard Ogden, and William Harwin. Can social interaction skills be taught by a social agent? the role of a robotic mediator in autism therapy. In Cognitive technology: instruments of mind, pages 57-74. Springer, 2001.

Kenton Williams and Cynthia Breazeal. Reducing driver task load and promoting sociability through an affective intelligent driving agent (aida). In IFIP Conference on Human-Computer Interaction, pages 619-626. Springer, 2013.

Margaret Wilson. Six views of embodied cognition. Psychonomic bulletin \& review, 9(4):625-636, 2002.

Robert A Wilson and Lucia Foglia. Embodied cognition. 2011. 
Jérémy Wrobel, Ya-Huei Wu, Hélène Kerhervé, Laila Kamali, Anne-Sophie Rigaud, Céline Jost, Brigitte Le Pévédic, and Dominique Duhaut. Effect of agent embodiment on the elder user enjoyment of a game. In ACHI 2013-The Sixth International Conference on Advances in ComputerHuman Interactions, 2013.

Keenan A Wyrobek, Eric H Berger, HF Machiel Van der Loos, and J Kenneth Salisbury. Towards a personal robotics development platform: Rationale and design of an intrinsically safe personal robot. In Robotics and Automation, 2008. ICRA 2008. IEEE International Conference on, pages 2165-2170. IEEE, 2008.

Steve Yohanan and Karon E MacLean. The role of affective touch in human-robot interaction: Human intent and expectations in touching the haptic creature. International Journal of Social Robotics, 4(2):163-180, 2012.

Tom Ziemke. Rethinking grounding. In Understanding representation in the cognitive sciences, pages 177-190. Springer, 1999.

Tom Ziemke. What's that thing called embodiment. In Proceedings of the 25th Annual meeting of the Cognitive Science Society, pages 1305-1310. Citeseer, 2003.

Jordan Zlatev. Situated embodiment studies in the emergence of spatial meaning. 1997.

Jakub Zlotowski. Comparison of robots' and embodied conversational agents' impact on users' performance. 2010 . 